\title{
WestVirginiaUniversity
}

THE RESEARCH REPOSITORY @ WVU

Graduate Theses, Dissertations, and Problem Reports

2012

\section{Three essays on political economy and development}

Adam Pellillo

West Virginia University

Follow this and additional works at: https://researchrepository.wvu.edu/etd

\section{Recommended Citation}

Pellillo, Adam, "Three essays on political economy and development" (2012). Graduate Theses,

Dissertations, and Problem Reports. 4908.

https://researchrepository.wvu.edu/etd/4908

This Dissertation is protected by copyright and/or related rights. It has been brought to you by the The Research Repository @ WVU with permission from the rights-holder(s). You are free to use this Dissertation in any way that is permitted by the copyright and related rights legislation that applies to your use. For other uses you must obtain permission from the rights-holder(s) directly, unless additional rights are indicated by a Creative Commons license in the record and/ or on the work itself. This Dissertation has been accepted for inclusion in WVU Graduate Theses, Dissertations, and Problem Reports collection by an authorized administrator of The Research Repository @ WVU.

For more information, please contact researchrepository@mail.wvu.edu. 


\title{
THREE ESSAYS ON POLITICAL ECONOMY AND DEVELOPMENT
}

\author{
Adam Pellillo \\ Dissertation submitted to the \\ College of Business and Economics \\ at West Virginia University \\ in partial fulfillment of the requirements \\ for the degree of
}

Doctor of Philosophy

in

Economics

\begin{abstract}
Andrew Young, Ph.D., Chair
Tami Gurley-Calvez, Ph.D.

Santiago Pinto, Ph.D.

Russell Sobel, Ph.D.

Christopher Coyne, Ph.D.

Department of Economics
\end{abstract}

Morgantown, West Virginia

2012

Keywords: Political Economy; Development; Conflict; Infant Mortality; Economic Reconstruction 


\section{Abstract \\ THREE ESSAYS ON POLITICAL ECONOMY AND DEVELOPMENT}

\section{Adam Pellillo}

This dissertation is a collection of three essays that examine how political economy and institutional factors influence development outcomes. In Chapter 1, I introduce each essay and argue that it is important to incorporate political economy and institutional considerations into studies in development economics. Chapter 2 examines the political economy of development in the Democratic Republic of the Congo by assessing the impact of conflict and local insecurity on household-level economic conditions across the country. Chapter 3 analyzes the political economy of infant mortality rates across the developing world by using an usually large microlevel dataset for 70 developing countries. Chapter 4 assesses the political economy of economic reconstruction in Afghanistan and Iraq by highlighting four 'reconstruction traps' that result from the incentives and constraints faced by those involved in reconstruction efforts. 


\section{Acknowledgements}

First and foremost, I would like to thank my parents, Vickie Mendenhall and Jay Pellillo, and my sister, Alex Pellillo, for their love and support.

I would also like to thank the members of my dissertation committee. Chris Coyne, Tami Gurley-Calvez, Santiago Pinto, Russ Sobel, and Andy Young each provided invaluable advice and encouragement throughout my graduate studies and the writing of this dissertation. I would like to thank Andy for his excellent guidance as my dissertation chair. Chris and Tami have been wonderful mentors, friends, and co-authors for more than four years and I am very fortunate to have them as colleagues.

Additional thanks to Elena Bondarenko, Amy Cheung, John Dove, Adam Hoffer, Maryam Naghsh, Chali Nondo, Hossein Radmard, Laura Seay, conference participants at the SEA (2010, 2011) and SRSA (2011) meetings, and seminar participants at CERGE-EI, DIW Berlin, the International School of Economics at Tbilisi State University (ISET), Montana State University - Billings, and West Virginia University for useful comments and suggestions on earlier drafts of the essays in this dissertation. Thanks to Filip Hilgert at IPIS for providing data on mining concessions and the MEASURE DHS team for answering my questions about the survey data. Wesley Shumway provided excellent research assistance.

I am also grateful to many colleagues in the Bureau of Business and Economic Research, the Department of Economics, and the WVU community for helpful conversations, feedback, and support during my time in Morgantown. In particular, I would like to thank Adam Hoffer for

being a wonderful friend, roommate, and co-author. Adam provided crucial feedback on all of my research endeavors, the job market process, and everything in between with remarkable patience and insight.

Lastly, I acknowledge generous financial support from the Department of Economics and the Charles G. Koch Doctoral Fellowship.

\section{Publication Information}

The third essay in this dissertation, "Economic Reconstruction Amidst Conflict: Insights from Afghanistan and Iraq," was published in the December 2011 issue of Defence and Peace Economics (co-authored with Christopher J. Coyne). This article is reprinted in this dissertation with the permission of the publisher (Taylor \& Francis Ltd, http://www.tandfonline.com). 


\section{Table of Contents}

Acknowledgements .........................................................................................................

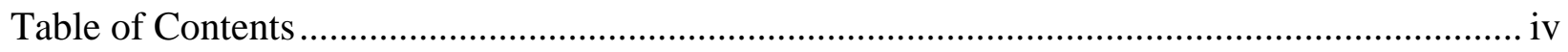

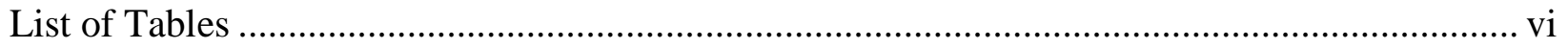

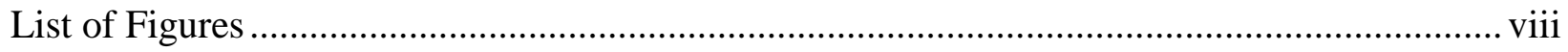

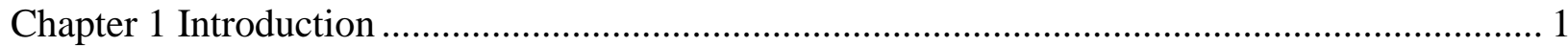

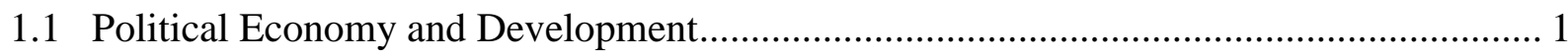

1.2 Conflict and Development: Evidence from the Democratic Republic of the Congo .......... 2

1.3 Infant Mortality over the Electoral Cycle ……………….................................................. 4

1.4 Economic Reconstruction Amidst Conflict: Insights from Afghanistan and Iraq .............. 5

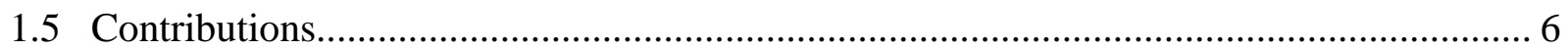

Chapter 2 Conflict and Development - Evidence from the Democratic Republic of the Congo ... 7

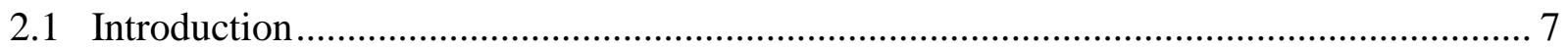

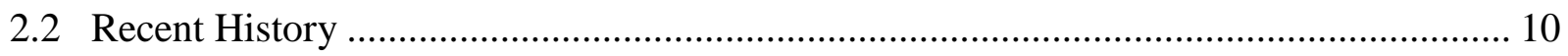

2.3 Data

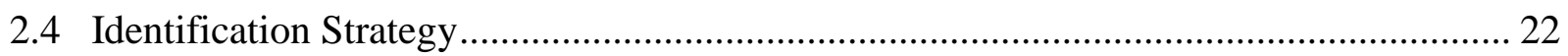

2.4.1 Potential Limitations ............................................................................................... 28

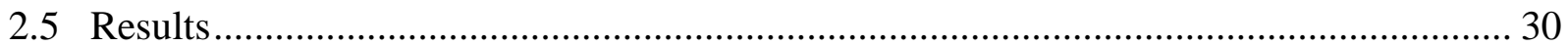

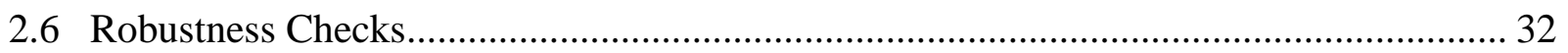

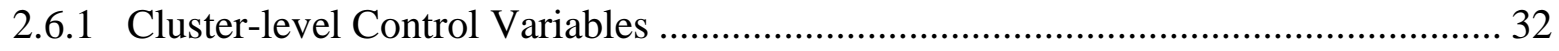

2.6.2 Different Measure of Household-level Wealth....................................................... 35

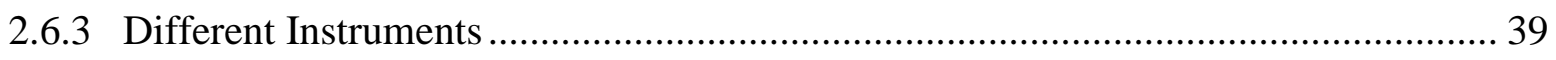

2.6.4 Splitting the Wealth Index into Two Components .................................................... 43

2.6.5 New Data on Conflict Events ........................................................................... 46

2.6.6 Different Measure of Conflict Exposure....................................................................... 50

2.6.7 Distance from Kinshasa ......................................................................................... 54

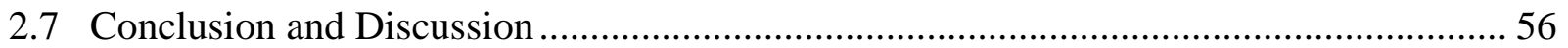

Chapter 3 Infant Mortality over the Electoral Cycle ................................................................. 58

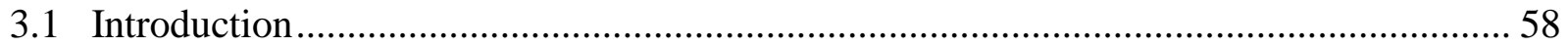

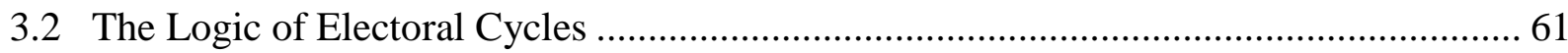


3.2.1 Electoral Incentives and Infant Mortality ................................................... 61

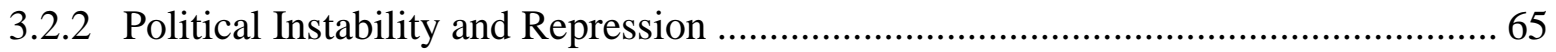

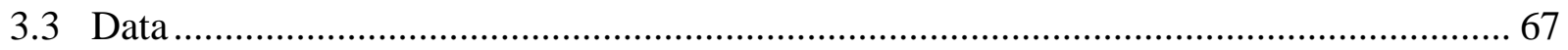

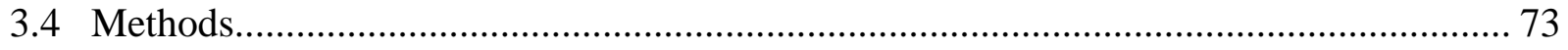

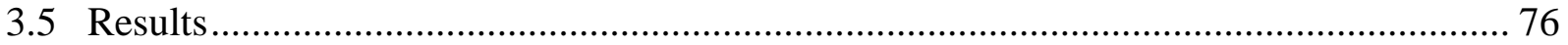

3.6 Timing of Election Dates and Infant Birth Dates ....................................................... 84

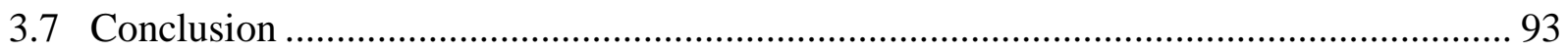

Chapter 4 Economic Reconstruction Amidst Conflict - Insights from Afghanistan and Iraq ..... 94

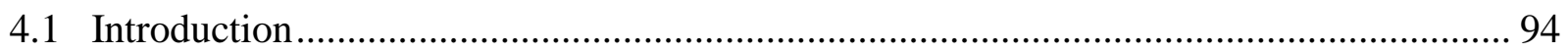

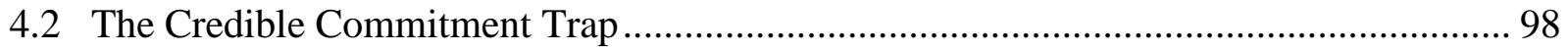

4.2.1 The Credible Commitment Problem ....................................................................... 99

4.2.2 Strategies for Avoiding the Credible Commitment Trap.................................... 102

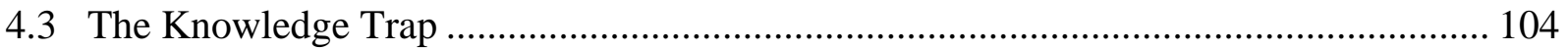

4.3.1 The Knowledge Problem ................................................................................. 106

4.3.2 Strategies for Avoiding the Knowledge Trap ............................................... 108

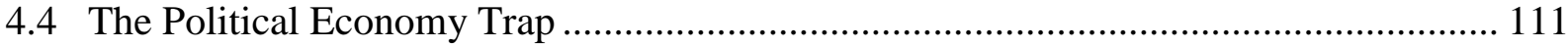

4.4.1 The Tradeoff Between Democracy and Economic Goals.................................. 112

4.4.2 Strategies for Avoiding the Political Economy Trap ........................................ 115

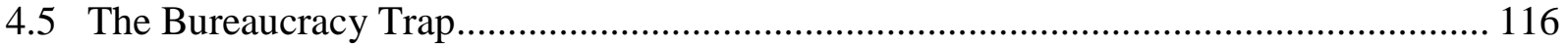

4.5.1 Bureaucracy and Economic Reconstruction ................................................. 117

4.5.2 Strategies for Avoiding the Bureaucracy Trap ............................................. 120

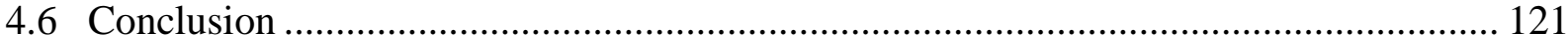

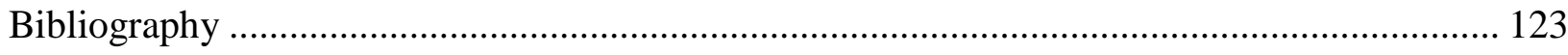

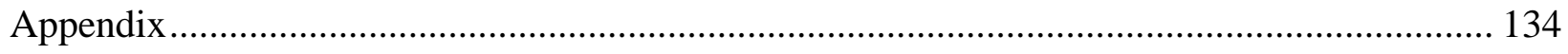




\section{List of Tables}

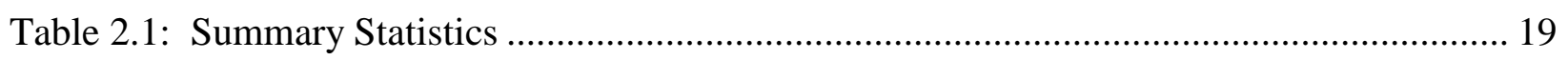

Table 2.2: Ordinary Least Squares Regression Results .................................................... 24

Table 2.3: Instrumental Variable Regression Results ...................................................... 31

Table 2.4: Summary Statistics -Variables Used in Section 2.6 .......................................... 34

Table 2.5: Instrumental Variable Regression Results - Inclusion of Cluster-level Control

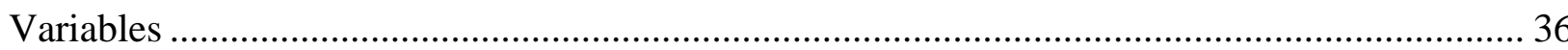

Table 2.6: Instrumental Variable Regression Results - Using the Original DHS Wealth Index . 38

Table 2.7: Instrumental Variable Regression Results with Latitude and Longitude Coordinates

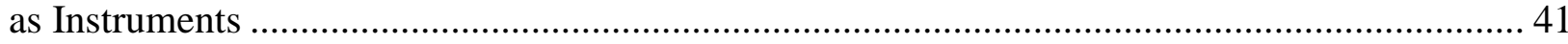

Table 2.8: Instrumental Variable Regression Results - New Instruments, DHS Wealth Index .. 42

Table 2.9: Instrumental Variable Regression Results - Wealth Index using "Liquid" Components

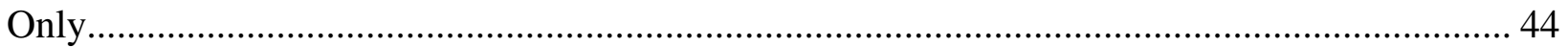

Table 2.10: Instrumental Variable Regression Results - Wealth Index using "Illiquid"

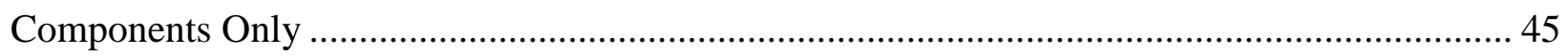

Table 2.11: Instrumental Variable Regression Results - UCDP-GED Data............................ 49

Table 2.12: Instrumental Variable Regression Results - ACLED Restricted (1) ..................... 52

Table 2.13: Instrumental Variable Regression Results - ACLED Restricted (2) ..................... 53

Table 2.14: Instrumental Variable Regression Results Using Distance from Kinshasa as a

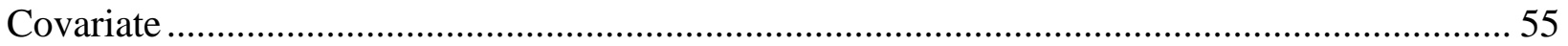

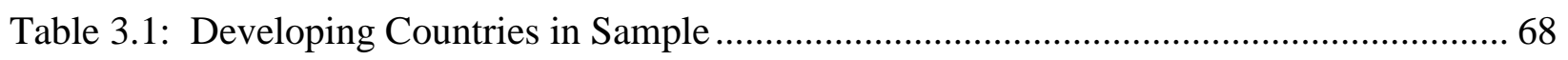

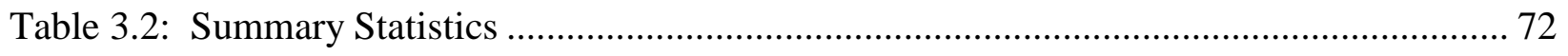

Table 3.3: Election Year Effects on Infant Mortality Rates ................................................ 77

Table 3.4: Election Year Effects on Infant Mortality Rates ............................................... 79

Table 3.5: Election Year Effects on Infant Mortality Rates - Interaction with Electoral

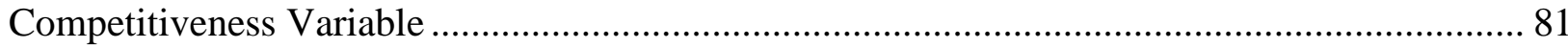

Table 3.6: Election Year Effects on Infant Mortality Rates - Interactions with System of

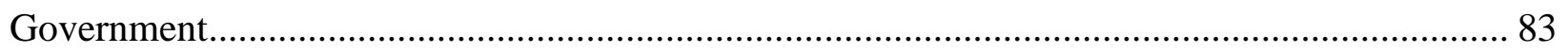

Table 3.7: Examples of Birth Months and Election Months and Definitions of Variables ......... 86

Table 3.8: In Utero Exposure to the Election Cycle ........................................................ 89 
Table 3.9: In Utero Exposure to the Election Cycle.

Table 3.10: In Utero Exposure to the Election Cycle - Interaction with Electoral

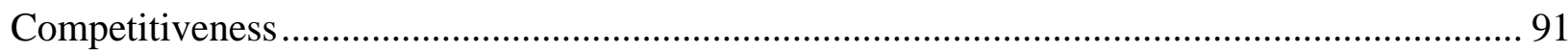

Table 3.11: In Utero Exposure to the Election Cycle - Interactions with Systems of Government 


\section{List of Figures}

Figure 2.1: Distribution (By Quintile) of Conflict Events (ACLED Data) .............................. 16

Figure 2.2: Distribution (By Quintile) of Cluster-level Average Household-level Wealth

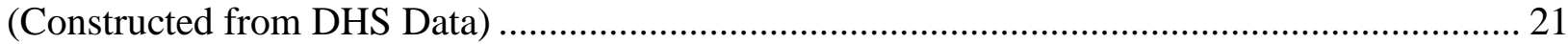

Figure 2.3: Scatterplots of Conflict Exposure (ACLED) and Distance from Goma ................. 26

Figure 2.4: Distribution (By Quintile) of Cluster-level Average Household-level Wealth (Using

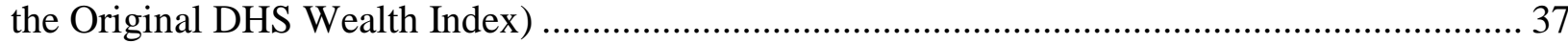

Figure 2.5: Scatterplots of Conflict Exposure (UCDP-GED) and Distance from Goma ........... 47

Figure 2.6: Distribution (By Quintile) of Conflict Events (UCDP-GED Data) ....................... 48 


\section{Chapter 1}

\section{Introduction}

\subsection{Political Economy and Development}

Economists have long studied the political economy of development. More than two and a half centuries ago, Adam Smith wrote that "little else is requisite to carry a state to the highest degree of opulence from the lowest barbarism but peace, easy taxes, and a tolerable administration of justice..." and that "all the rest are [brought] about by the natural course of things" (Smith, 1776: xl). Yet the roles played by political economy and institutional factors in the process of economic development remain understudied by scholars, policymakers, and aid professionals. While Smith's insights into the political economy of development are powerful ones, many questions in this research area remain unanswered. Further, many of the standard 'answers' in the literature (e.g., Acemoglu, Johnson and Robinson [2001] on the role of institutions in explaining cross-country variation in economic performance) are the subject of considerable debate. ${ }^{1}$

The goal of this dissertation is to contribute to the fast-growing literature on political economy and development by examining how specific political economy and institutional factors influence economic and health outcomes across the developing world. To this end, I introduce political economy considerations into the analysis of household living conditions and durable ownership, infant mortality rates across countries, and externally-led efforts at economic

\footnotetext{
${ }^{1}$ For instance, Glaeser, La Porta, Lopez-de-Silanes, and Shleifer (2004) argue that human capital matters more for growth than institutions and that institutions are actually the outcome of better policies and economic growth.
} 
reconstruction and state building. ${ }^{2}$ All too often, each of these topics is approached without a consideration of political economy constraints or the incentives faced by different political actors. It is highly worthwhile to test whether there are political economy factors that shape important economic and health outcomes (like household-level wealth, infant mortality, or even entire economic institutions). In what follows, I introduce the three essays in this dissertation and discuss their main findings and implications.

\subsection{Conflict and Development: Evidence from the Democratic Republic of the Congo}

Much of the recent literature on the political economy of development has focused on how cross-country variation in the protection of private property rights or rule of law accounts for why some countries are rich while other countries are poor. ${ }^{3}$ Empirically, those countries with poorly enforced private property rights have worse development outcomes than those countries with relatively more secure property rights (Acemoglu and Johnson, 2005; Acemoglu, Johnson, and Robinson, 2005). The same is true for economic freedom: those countries that are relatively more economically free have higher rates of economic growth and higher levels of income per person (see Chauffour, 2011 for a review of this literature).

Much of this literature focuses on the role of institutions in explaining cross-country variation in economic performance in a large- $\mathrm{N}$ framework. Increasingly, economists are exploring the implications of within-country variation in property rights protection for economic performance.

\footnotetext{
${ }^{2}$ This follows Acemoglu's (2010) call for the inclusion of political economy factors in inquiries into development problems and Besley and Persson's (2011) contributions on the political economy of development.

${ }^{3}$ Of course, economists have long focused on the importance of private property rights for economic growth and development (see, for instance, Smith [1776] and Mises [1920]).
} 
The first essay in this dissertation examines how conflict exposure has influenced household asset ownership and living conditions across the Democratic Republic of the Congo (DRC). ${ }^{4}$ The DRC has experienced ongoing political instability and violent conflict for almost two decades, yet the causes and economic consequences of conflict in the DRC have received relatively little attention from the academic community. ${ }^{5}$ I employ micro-data from the 2007 Demographic and Health Survey (DHS) for the DRC along with disaggregated and georeferenced information on regional conflict exposure from the Peace Research Institute Oslo (PRIO) to examine the microeconomic consequences of conflict in the DRC.

Because conflict events were not randomly assigned across the country, I use two different instrumental variables approaches, both which rely on plausibly exogenous geographic and historical factors for identification. In line with many qualitative reports on the topic, I find evidence of a strong, negative impact of conflict exposure on household-level economic outcomes in the DRC. This finding is robust to the inclusion of different control variables, definitions of conflict exposure and household-level 'wealth,' and sample restrictions. The results indicate that political economy factors - here, ongoing political instability and violent conflict - have had substantial impacts on development outcomes across the country. This paper contributes to the literature on the influence of the rule of law and security of property rights by identifying how regional differences in the degree to which property is secure from expropriation or exposure to ongoing conflict influence regional economic outcomes.

\footnotetext{
${ }^{4}$ See Figure A.1 in the Appendix.

${ }^{5}$ Because most economic data from the DRC are highly unreliable, there have only been a few studies that address development outcomes within the country.
} 


\subsection{Infant Mortality over the Electoral Cycle}

Recently, a number of studies have shown that infant mortality rates across the developing world are highly sensitive to fluctuations in aggregate income, changes in weather patterns and disease ecology, and commodity prices. To date, only a few studies have explored whether changes in political economy factors similarly influence infant mortality. In essence, we have a growing body of evidence regarding the importance of economic and environmental 'shocks' for infant mortality rates, but there is less of an understanding of how political economy 'shocks' similarly affect infant mortality rates.

The second essay in this dissertation introduces political economy 'shocks' into the analysis of infant mortality fluctuations. Specifically, this essay tests whether infant mortality rates fluctuate in the vicinity of elections. This article therefore contributes to our understanding of the political economy of infant mortality rates and expands the literatures on political business cycles and political budget cycles by considering for the first time whether infant mortality rates similarly fluctuate along the electoral cycle.

To answer this question, I use a large panel (more than 2 million observations) of individual-level indicators of infant mortality and other covariates. Using a variety of specifications, I find only limited evidence of electoral cyclicality in infant mortality rates. This result holds even when considering the heterogeneous timing of elections and infant birth dates. Ultimately, while it is likely that there is electoral cyclicality in transfer payments and government expenditures in a number of developing countries, there is only limited evidence of an impact of elections on infant mortality rates. 


\subsection{Economic Reconstruction Amidst Conflict: Insights from Afghanistan}

\section{and Iraq}

As Buchanan (1975) argued in The Limits of Liberty, the core responsibilities of the state are to enforce constitutional order (the 'protective' role of the state) and provide public goods (the 'productive' role of the state). Yet all too often states fulfill neither of these roles and instead engage in what Buchanan called the 'predatory' functions of the state. Where states are characterized by persistent weakness or state institutions have entirely collapsed (e.g., in places like Afghanistan, the Democratic Republic of the Congo, Somalia, etc.), public goods like security and the rule of law are systematically underprovided. Sub-national non-state armed groups maintain considerable control over different regions of a country and often operate with impunity. ${ }^{6}$ The national armies in these countries are also poorly controlled and in places like the Democratic Republic of the Congo we observe human rights abuses by members of these armies.

It is well-acknowledged that state failure has severe consequences for an array of economic and health outcomes. Rotberg (2004: 6) argues the following:

In most failed states, regimes prey on their own constituents. Driven by ethnic or other intercommunal hostility, or by the governing elite's insecurities, they victimize their own citizens or some subset of the whole that is regarded as hostile. As in Mobutu Sese Seko's Zaire or the Taliban's Afghanistan, ruling cadres increasingly oppress, extort, and harass the majority of their own compatriots while privileging a more narrowly based party, clan, or sect.

Taking this political status quo as given, many policymakers have sought solutions to these pressing problems. Yet state building and economic reconstruction are time- and resourceintensive endeavors that are beset by many problems of informational limitations and incentive compatibility (Coyne and Pellillo, 2011a).

\footnotetext{
${ }^{6}$ Then again, in places like Somaliland, the quasi-governments that have emerged actually fulfill the protective and productive roles of the state better than in other areas of the country (e.g., Mogadishu).
} 
The last essay in this dissertation applies the tools of public choice economics to economic reconstruction and state building efforts in Afghanistan and Iraq. Specifically, the incentives and constraints faced by those involved in these processes are mapped out and four 'reconstruction traps' are identified. The 'traps' are much like others that are discussed in the literature (e.g., 'poverty traps,' 'conflict traps,' etc.) but are more oriented toward the potential policy traps in which the international community often becomes entangled. These reconstruction traps include (1) the credible commitment trap, (2) the knowledge trap, (3) the political economy trap, and (4) the bureaucracy trap. A number of examples and insights from Afghanistan and Iraq are used to motivate and elucidate the reconstruction traps.

While the consequences of state failure or state weakness for development outcomes are severe (as illustrated in the first essay of this dissertation), these reconstruction traps suggest that the capacity for the international community to intervene and 'fix' failed states is limited.

\subsection{Contributions}

Each of these three essays introduces political economy and institutional considerations into the analysis of development processes and outcomes. Given the consequences of political economy and institutional factors for development, more research in this field is crucially needed. 


\section{Chapter 2}

\section{Conflict and Development - Evidence from the Democratic Republic of the Congo}

\subsection{Introduction}

In the wake of the 1994 Rwandan genocide, the Democratic Republic of the Congo (DRC) experienced two devastating civil wars. The $2^{\text {nd }}$ civil war in particular was associated with the deaths of millions of civilians, widespread internal displacement, severe human rights abuses, and the destruction of infrastructure, homes, and physical and human capital. ${ }^{7}$ During the civil wars, schools and hospitals were attacked by rebel groups and capital assets were expropriated by armed groups and government troops (Global Witness, 2009; OHCHR, 2010). In the eastern province of North Kivu, over the course of three years following the Rwandan genocide, it is estimated that up to 80 percent of the livestock were pillaged by rebel groups (OHCHR, 2010). Civil conflict and attacks on civilians continue to date, particularly in the eastern and northeastern provinces of the country.

While a number of studies have discussed some of the economic consequences of conflict in the DRC (Nzongola-Ntalaja, 2002; Nest et al., 2006; Turner, 2007; Global Witness, 2009; Prunier, 2009; OHCHR, 2010; Autesserre, 2010, Stearns, 2011), to date there has not been a systematic empirical analysis of the impact of conflict on economic conditions across the country. This paper expands the literature on the topic by examining how conflict events (i.e., 'battles' or 'attacks') have influenced household-level measures of durable ownership and living

\footnotetext{
${ }^{7}$ Most of these civilian deaths were related to malnutrition, public health crises, and general economic decline (Turner, 2007). The International Rescue Committee (IRC, 2004) estimates a death toll of nearly 3.8 million for the second civil war alone, though this estimate is the subject of debate (Human Security Report Project [2010]).
} 
conditions across the country. More generally, this paper assesses the importance of local and regional political economy factors for economic development. It is well-acknowledged that the rule of law and security of person and property are crucial for economic growth and development (Haggard and Tiede, 2011). Yet few papers explicitly focus on the implications of local and regional variation in the support for the rule of law or the security of person and property for household-level economic outcomes.

While there is an extensive literature on the country-level institutional determinants of economic growth and development (see, among others, Acemoglu, Johnson, and Robinson, 2001; Rodrik et al., 2004; Acemoglu and Johnson, 2005; Haggard and Tiede, 2011), there is also a fast-growing literature on the local and regional political economy of microeconomic outcomes. Recent studies of the microeconomic consequences of conflict address the impact of conflict and political instability on human capital accumulation (Akresh and de Walque, 2010; Shemyakina, 2011; Chamarbagwala and Morn, 2011), local institutions (Bellows and Miguel, 2006; 2009), political participation (Blattman, 2009; Kyle, 2010), labor market outcomes (Kondylis, 2010), and health outcomes (Bundervoet, Verwimp, and Akresh, 2009; Akresh, Verwimp, and Bundervoet, 2011; Akresh, Bhalotra, Leone, and Osili, 2011; Minoiu and Shemyakina, 2012).

The importance of understanding the implications of conflict and insecurity for development cannot be understated. While the impact of conflict on development in the DRC may appear straightforward-Collier et al. (2003), for instance, refer to civil war as 'development in reverse'- there is reason to believe the empirical relationship between conflict events and economic outcomes may be somewhat ambiguous. Consider that Hegre, Østend, and Raleigh (2009) find that conflict events during the 1989-2002 Liberian civil war took place more 
frequently in relatively wealthier areas of the country, a result that is consistent with the results of Collier and Hoeffler (2004) which suggest that 'rebel opportunity' partially explains why civil war occurs. Blattman and Miguel (2010) also point out that while the correlation between low per capita incomes and the onset of conflict is one of the strongest in the empirical literature on conflict, it is highly likely the relationship between the two moves in both directions: low incomes may set the underlying conditions that increase the likelihood of conflict (perhaps because of weak state capacity, as argued by Fearon and Laitin, 2003, or because of rebel opportunity, as argued by Collier and Hoeffler, 2004), yet conflict is also associated with the destruction of resources, loss of life, and widespread displacement. ${ }^{8}$ These studies suggest that OLS estimates of the impact of conflict on development in the DRC may be biased.

To shed light on the impact of conflict and local insecurity on economic conditions across the DRC, I combine household-level data on durable ownership and living conditions from the 2007 Demographic and Health Surveys (DHS) with disaggregated and geo-referenced conflict data from the Armed Conflict and Location Event Dataset (ACLED). Because conflict events were not randomly assigned across the country, I use the variation in distances between DHS cluster locations (the latitude and longitude coordinates at which surveys were administered) and Goma, a city along the DRC-Rwanda border, as an instrument for conflict exposure. This instrument accounts for the fact that conflict events have taken place at a much higher rate in the eastern and northeastern provinces. Accounting for the distribution and intensity of conflict events across the country helps to isolate the effect of conflict on development in the DRC, yet this approach is not without its limitations. In Section 6, I discuss different strategies for identifying the relationship between conflict and development.

\footnotetext{
${ }^{8}$ Do and Iyer (2010) also examine violent conflict in Nepal, finding an association between high levels of poverty and conflict intensity.
} 
I find that the conflict events that have taken place in the DRC have had a significant negative impact on household-level economic outcomes. Violent political activity is associated with strong reductions in durable ownership and household living conditions. These results are similar when different specifications and variable definitions and sources are considered. The findings illustrate the importance of understanding the incentives faced by different political actors (e.g. governments, rebel organizations, local militias, citizens, etc.) in 'weak' and 'fragile' states.

The remainder of this paper proceeds as follows. Section 2 discusses the recent economic and political history of the DRC. Section 3 describes the data and Section 4 presents the identification strategy. Section 5 presents the results while Section 6 assesses their robustness to alternative specifications, variable definitions, and variable sources. Section 7 concludes.

\subsection{Recent History}

In this section, I summarize the recent political and economic history of the DRC. To be certain, the extraction-oriented institutions that were established under King Leopold II, the country's history of colonial rule by Belgium, and post-independence political instability played substantive roles in shaping the political and economic status quo we observe today. Yet I focus primarily on the most recent political and economic history of the DRC in order to establish the context for the empirical analysis in the following sections.

Prior to the overthrow of President Mobutu Sese Seko's regime (which lasted from 19651997), the economic and political status quo in the DRC (then Zaire) was characterized by weak constraints on executive authority, endemic corruption and extensive patronage networks, hyperinflation, low agricultural and industrial productivity, and weak state financial capacity 
(Prunier, 2009). Mobutu's rule was associated with sharp economic decline and the deterioration of state institutions.

In the early 1990s, Mobutu's regime and patronage network declined rapidly, particularly when Cold War-era economic and military aid from the United States came to a near stop. ${ }^{9}$ Land disputes and conflicts related to citizenship rights (which resulted from legal changes in Kinshasa) erupted during this time period, particularly in the eastern provinces of the country (Autesserre, 2010). The expropriation of household durables also began during this time period. Autesserre (2010: 56) describes how "instead of paying his armed forces, Mobutu encouraged them to remunerate themselves through looting the scapegoats' properties-in addition to conducting clandestine trade and attacking humanitarian aid assets." The ineffectiveness of the state was particularly evident in the eastern provinces of the DRC. Prior to his regime's collapse, in the 1980s Mobutu cut expenditures on public goods (e.g. health care, education, and infrastructure) in the region, although tax rates remained high (Autesserre, 2010: 70). ${ }^{10}$

During this period of economic and institutional decline in Zaire, the Rwandan genocide took place in the spring of 1994. The genocide-perpetrated by génocidaires like the Interahamwe, the Hutu Power organization, and members of the former Forces Armées Rwandaises (FAR) - took the lives of more than 800,000 Tutsis and moderate Hutus. The Rwandan Patriotic Front (RPF) invaded from Uganda to seize the Rwandan capital, Kigali, and to stop the genocide. Many of the génocidaires took cover in refugee camps alongside more than one million refugees from Rwanda (and Burundi) that were situated in the eastern provinces of

\footnotetext{
${ }^{9}$ Remarkably, almost all U.S. economic and military aid to Zaire came to a stop at the end of the Cold War. Over the previous decade, the U.S. had provided Mobutu with hundreds of millions of dollars in economic and military aid (close to $\$ 991$ million in the 1980s alone). No longer useful geopolitically toward the end of the Cold War, the United States even imposed sanctions on Mobutu's regime to induce democratization. Mobutu responded accordingly, holding the first multiparty elections in the country in the early 1990 s.

${ }^{10}$ This is one of the motivations for using 'education in years' as a control variable in selected specifications below.
} 
the DRC along the DRC-Rwanda border. In September 1996, General Kagame of Rwanda sent a force supported by the Rwandan Patriotic Army (RPA) (formerly the RPF) into Zaire with the intent of neutralizing the génocidaires. The RPA did so in conjunction with the Uganda People's Defense Force (UPDF) and other political organizations, which eventually led to the formation of the Alliance des Forces Démocratiques pour la Libération du Congo-Zaïre (AFDL).

Yet along with the desire to counter the génocidaires, there was also a broader goal of overthrowing Mobutu's regime, mainly because of his reticence in countering armed groups that took refuge in some parts of the country. Prior to the invasion, the heads of state from Angola, Eritrea, Ethiopia, Rwanda, Uganda, and Zimbabwe were involved in plans for regime change (Prunier, 2009: 67). As Laurent-Désiré Kabila, the leader of the AFDL, marched across Zaire from the east, Mobutu and his top officers fled the country. Kabila arrived in Kinshasa on May 20, 1997, ending the first civil war, and sought to establish political control over the capital. Kabila subsequently renamed the country from Zaire to the Democratic Republic of the Congo.

However, political alliances quickly changed. Kabila was no longer willing to attack the génocidaires and other groups Uganda and Rwanda perceived as threats. With Uganda and Rwanda no longer in support of Kabila, they sent troops, arms, and money into Zaire with the intent of toppling Kabila's regime. Rebels backed by the RPA commandeered a cargo plane in Goma and flew to the Atlantic Coast to launch a military campaign (Nest et al., 2006: 25). ${ }^{11}$ With the splintering of rebel groups, the development of new alliances (e.g. many Interahamwe and ex-FAR troops jointed the Forces Démocratiques de Libération du Rwanda [FDLR] and Uganda backed the Mouvement de Libération du Congo [MLC] and a faction of the RCD [different than the faction supported by Rwanda]) and the involvement of neighboring countries such as Angola, Chad, Eritrea, Namibia, Rwanda, Sudan, Uganda, and Zimbabwe, the second civil war continued

\footnotetext{
${ }^{11}$ This explains why some of the conflict events took place near the DRC's capital, Kinshasa (see below).
} 
until mid-2003, when the Transitional Government in Kinshasa (the 'Government of National Unity and Transition') was established after a series of peace agreements and negotiations. ${ }^{12}$ The Transitional Government was comprised by some of the most powerful political groups, including Kabila's former government and rebel movements-turned-political parties (e.g. the Ugandan-allied MLC, Mai Mai militias, and different Ugandan- and Rwandan-backed factions of the Rassemblement Congolais pour la Démocratie [RCD], etc.) (Autesserre, 2010). The second civil war left a devastating death toll, with millions of casualties resulting mainly from malnutrition, public health crises, and generally poor living standards (IRC, 2004).

While the transition to the new government occurred almost a decade ago, various armed groups, local militias, and the Congolese national army (FARDC) are still fighting in the eastern provinces of the DRC. Many armed groups continue to abuse citizens in these provinces, charging 'taxes,' engaging in atrocious human rights abuses and rapes, and expropriating household assets and consumer durables. ${ }^{13}$

The conflict events that continue to take place in the eastern provinces appear to center on disputes over natural, mineral, and land resources, though both the fundamental and proximate causes of the conflict events are complex and the source of considerable debate (Stearns, 2011). Nest et al. (2006: 12-13) contend that the conflict events that have taken place in the country are mainly due to the weakness of the state, military, financial, and logistic interventions from neighboring countries, ethnic political violence, regime survival, and contests for control over natural and mineral resources. ${ }^{14}$ They hold that the structural conditions for

\footnotetext{
${ }^{12}$ Prior to the establishment of the Transitional Government, Kabila was assassinated by his bodyguard. His son, Joseph Kabila, subsequently took over political power.

${ }^{13}$ As succinctly described by Autesserre (2010: 4), "throughout the transition, unremitting clashes between various armed groups and militias, frequent massacres of civilians, massive population displacements, and appalling human rights violations, including widespread sexual violence, persisted in the provinces of North Kivu, South Kivu, North Katanga, and in Oriental Province's Ituri district.”

${ }^{14}$ The civil wars in the DRC involved up to fourteen foreign armies at different time periods (Autesserre, 2010: 2).
} 
conflict are ultimately rooted in the weak political and legal institutions first established under Belgian colonial rule. Along with seeking to maintain political power through 'divide-and-rule' strategies, "the DRC state and individual officials also sought to systematically extract revenue for private and institutional purposes" and "it is under these conditions that ethnic and regional grievances and conflicts over access to natural resources have long flourished" (Nest et al., 2006: $13)$.

Yet the conflict events may also be rooted in local political agendas and incentives. Autesserre (2010) argues that these factors have influenced the conflict dynamics of the civil wars and the current civil unrest in the eastern provinces of the country. As Autesserre (2010: 8) notes that, "these [local] agendas pitted villagers, traditional chiefs, community chiefs, or ethnic leaders against one another over the distribution of land, the exploitation of local mining sites, the appointment to local administrative and traditional positions of authority, the collection of local taxes, and the relative social status of specific groups and individuals." Garrett and Seay (2011: 85) argue that "the primary basis of conflict in the Kivu provinces is longstanding tension over ethnicity, citizenship rights and land rights, which are in turn related to grievances over access to resources such as land, and over legitimacy and power. Conflict dynamics also include the marginalization of eastern DRC borderland areas from the capital Kinshasa, which are themselves symptomatic of broader governance failures in the DRC."

It is crucial to understand which factors matter most for the continuation of conflict in the eastern provinces, particularly considering that contemporary policy measures (e.g., Section 1502 of the Dodd-Frank Wall Street Reform and Consumer Protection Act) are formulated on the basis of this understanding. In what follows, I put forth an instrumental variable regression approach to endogenize the locations of violent political activity across the country and then, in 
turn, examine how conflict and local insecurity have influenced household-level durable ownership and living conditions. ${ }^{15}$

\subsection{Data}

To examine the microeconomic consequences of conflict, I combine disaggregated and georeferenced conflict event data from the Armed Conflict and Location Event Dataset (ACLED) with household-level data on durable ownership and living conditions from the 2007 cross sectional Demographic and Health Survey (DHS) for the DRC.

The Centre for the Study of Civil War (CSCW) at the Peace Research Institute Oslo (PRIO) has developed the Armed Conflict and Location Event Dataset (ACLED), which provides information on each specific conflict event for the DRC. The DRC ACLED data contain exact dates, locations (at the latitude and longitude coordinate), and additional characteristics of conflict events during the first and second civil wars (data from 1997 to 2004) as well as the current conflict events that have taken place largely in the eastern provinces of the DRC (from 2004 until 2007 for the analysis here). These events were recorded on the basis of reports from war zones, humanitarian agencies, and other research publications. Figure 2.1 presents a graphical depiction of the geographic distribution and intensity of conflict events across the DRC.

\footnotetext{
${ }^{15}$ The relationship may be most evident in the eastern provinces of the Kivus and Oriental Province, where "more than $80 \%$ of the inhabitants of these places consider their living conditions to be the same as or worse than during the wars" (Autesserre, 2010: 4).
} 


\section{Figure 2.1: Distribution (By Quintile) of Conflict Events (ACLED Data)}

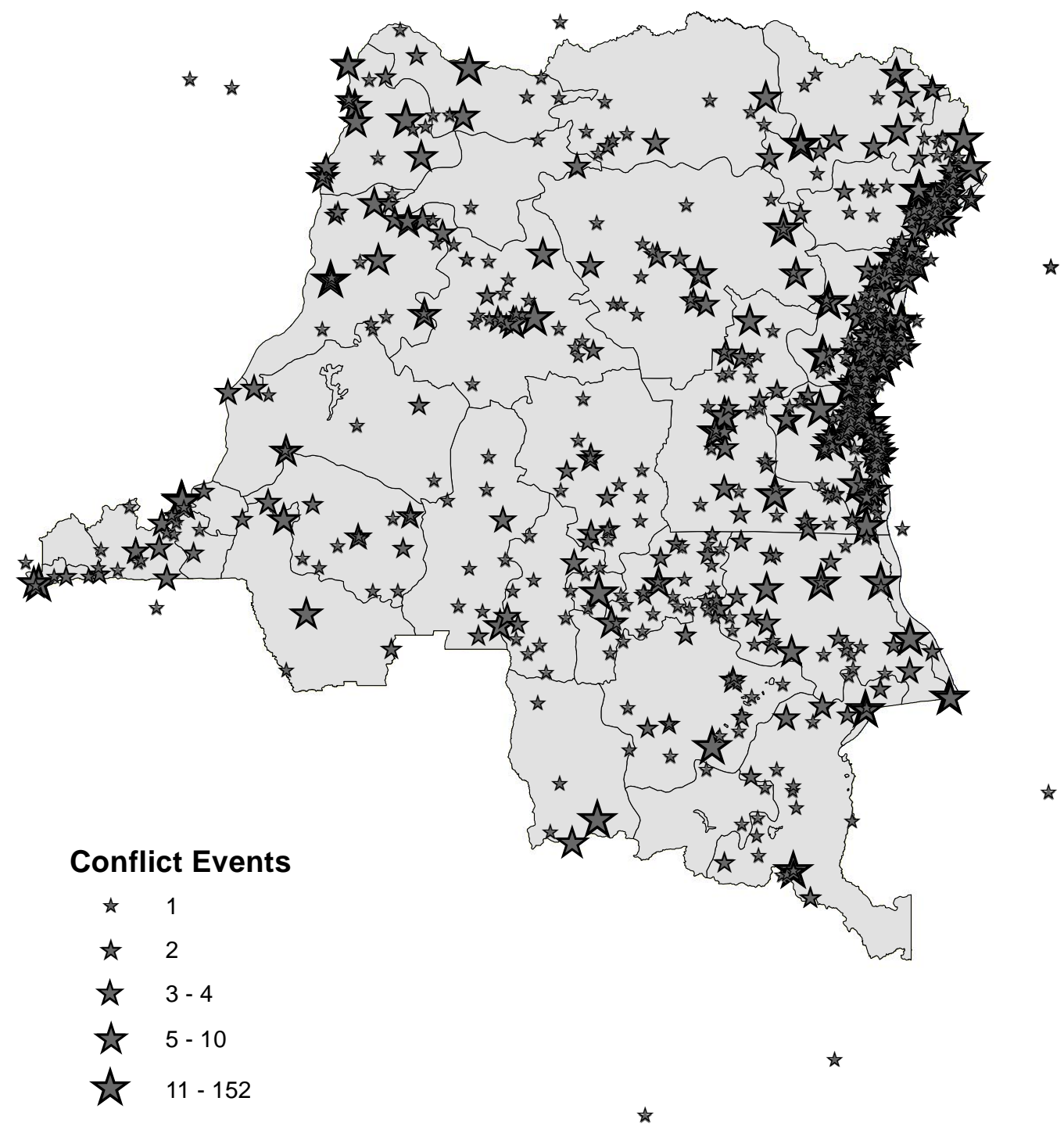

The Demographic and Health Survey (DHS) program (also known as MEASURE DHS) was initially established by USAID in 1984 . The DHS surveys provide nationally representative 
individual- and household-level data on a range of socio-economic and health conditions for a number of countries across the developing world. Surveys are implemented in each country by ICF Macro in collaboration with the relevant administrative organizations in participating countries. ${ }^{16}$ Because the DHS surveys are primarily designed with the goal of collecting information on marriage, fertility, family planning, reproductive health, child health, and HIV/AIDS, women of reproductive age (15-49) are the primary focus of the DHS surveys. Yet the primary survey data are recoded in a number of formats, including household-level and individual-level recode files.

In the DRC, surveys were administered at 300 cluster locations across the country. Each cluster location has a unique latitude and longitude coordinate assigned to it. In order to ensure the confidentiality of survey respondents, MEASURE DHS randomly displaces the GPS latitude/longitude coordinate positions for all of their surveys. Specifically, for each urban cluster, they ensure a minimum of 0 and maximum of 2 kilometers of positional error. For each rural cluster, there is a minimum of 0 and maximum of 5 kilometers of positional error. Some of the rural clusters that are easily identifiable (about $1 \%$ of the total) are displaced up to 10 kilometers. To develop household-level economic data for use in this paper, I employ the household-level recode file for the DRC and combine it with latitude and longitude coordinates for each DHS cluster. ${ }^{17}$ The data are representative at the national level, for residents in both

\footnotetext{
${ }^{16}$ USAID provides financial support for many of the surveys, though other countries receive funding from donors like UNICEF, UNFPA, and the World Bank (Rutstein and Royas, 2006).

${ }^{17}$ In order to isolate the impact of conflict on household-level wealth, I only use the data points that have latitude and longitude coordinates, which include 2,990 of 2,999 conflict events and 8,679 out of 8,886 household-level observations. There are 3,959 conflict events in total in the ACLED file for the DRC, yet I only consider those conflict events that took place prior to 2007 in order to match these data with the household-level economic information. I confirmed with ICF Macro that the DHS clusters for the 2007 DRC survey that were missing geomarkers were not missing this information because of security concerns in these areas. The latitude and longitude coordinates for these 7 clusters (out of 300) were not able to be verified due to errors from the GPS receivers (e.g., satellite acquisition errors). The omission of these specific clusters will not introduce any bias into the results. The
} 
rural and urban areas, and in all eleven provinces of the country (Kinshasa, Bas-Congo, Bandundu, Équateur, Orientale, Nord-Kivu, Sud-Kivu, Maniema, Katanga, Kasaï Oriental, and Kasaï Occidental).

'Conflict exposure' is the key explanatory variable of interest in this paper. I construct various measures of 'conflict exposure' based on the number of conflict events occurring within set distances $(200 \mathrm{~km}, 100 \mathrm{~km}$, and $50 \mathrm{~km})$ of DHS cluster locations. These distances are chosen not only because of the sheer size of the country (see summary statistics in Table 2.1 and Table 2.4) but also because the latitude and longitude coordinates are not at the individual household level but at the DHS cluster locations. ${ }^{18}$ Further, because of the random displacement of GPS latitude and longitude coordinate positions in the DHS GPS file, it is necessary to use a broad radius around DHS cluster locations to capture the degree to which these cluster locations were exposed to violent political activity. These measures of conflict exposure should sufficiently capture the effects of local and regional violent political activity, local insecurity, poor rule of law, and the direct impact of the conflict events (i.e., 'battles' or 'attacks') themselves.

individual recode file used in this analysis as well as the GPS data are available (with prior approval) at the DHS site, http://www.measuredhs.com.

${ }^{18}$ To calculate measures of conflict exposure, I first convert the latitude and longitude coordinates of both the DHS micro-level wealth data and the ACLED disaggregated conflict data from degrees to radians. This entails multiplying each latitude and longitude coordinate by $\frac{2 \pi}{360}$. I then take the differences between latitude and longitude coordinates for the DHS and ACLED data and calculate the Haversine formula for the central angle for a distance measure: $\quad A=\sin \left(\frac{\Delta L a t}{2}\right)^{2}+\cos \left(L_{a} t_{A C L E D}\right) * \cos \left(L a t_{D H S}\right) * \sin \left(\frac{\Delta L o n g}{2}\right)^{2} . \quad$ I then calculate $2 * \operatorname{atan} 2(\operatorname{sqrt}(A), \operatorname{sqrt}(1-A))$ and then multiply this figure by the earth's radius in kilometers $(6371 \mathrm{~km})$ to derive the final distance measure. To ensure that this method is accurately capturing cluster-level conflict exposure, I have also calculated measures of conflict exposure using the ArcGIS buffer command, where I created 200km, $100 \mathrm{~km}$, and $50 \mathrm{~km}$ buffers around the DHS cluster locations and counted the number of conflict events that took place within each buffer zone. 


\section{Table 2.1: Summary Statistics}

\begin{tabular}{|c|c|c|c|c|c|}
\hline & \# Obs. & Mean & Std. Dev. & Min & Max \\
\hline Wealth Index & 8435 & 5.021814 & 2.93794 & 0 & 16 \\
\hline Television & 8435 & .1481921 & .3553113 & 0 & 1 \\
\hline Refrigerator & 8435 & .0456432 & .2087224 & 0 & 1 \\
\hline Motor Transport & 8435 & .0284529 & .1662726 & 0 & 1 \\
\hline Telephone & 8435 & .0054535 & .0736503 & 0 & 1 \\
\hline Electricity & 8435 & .165738 & .3718673 & 0 & 1 \\
\hline Radio & 8435 & .4519265 & .4977131 & 0 & 1 \\
\hline Bicycle & 8435 & .2162418 & .4117055 & 0 & 1 \\
\hline Cement Floor & 8435 & .2061648 & .4045742 & 0 & 1 \\
\hline Improved Water Source & 8435 & .4704209 & .4991539 & 0 & 1 \\
\hline Mobile Phone & 8435 & .2256076 & .4180066 & 0 & 1 \\
\hline Grill/Heater & 8435 & .0994665 & .2993051 & 0 & 1 \\
\hline Chairs & 8435 & .7346769 & .4415313 & 0 & 1 \\
\hline Beds & 8435 & .7882632 & .4085635 & 0 & 1 \\
\hline Lamps & 8435 & .6628334 & .4727703 & 0 & 1 \\
\hline Stove/Cooker & 8435 & .0266746 & .1611401 & 0 & 1 \\
\hline Hoes & 8435 & .6701838 & .4701741 & 0 & 1 \\
\hline Improved Toilet & 8435 & .0758743 & .2648126 & 0 & 1 \\
\hline Number in Household & 8435 & 5.433432 & 3.011624 & 1 & 28 \\
\hline Capital or Large City & 8435 & .25738 & .4372164 & 0 & 1 \\
\hline Small City & 8435 & .0235922 & .1517838 & 0 & 1 \\
\hline Town & 8435 & .1353883 & .3421581 & 0 & 1 \\
\hline Altitude & 8435 & 6.420025 & .7063543 & 3.044523 & 8.188967 \\
\hline Education (in years) of $\mathrm{HHH}$ & 8435 & 6.704327 & 4.766502 & 0 & 18 \\
\hline Percent of $\mathrm{HH}$ with Under 5 & 8435 & .1881321 & .178953 & 0 & .8 \\
\hline Male HHH & 8435 & .7892116 & .4078926 & 0 & 1 \\
\hline Age of $\mathrm{HHH}$ & 8435 & 42.98791 & 14.35795 & 13 & 95 \\
\hline Conflict Exposure - $200 \mathrm{~km}$ & 8435 & 229.5911 & 279.8332 & 8 & 973 \\
\hline Conflict Exposure - $100 \mathrm{~km}$ & 8435 & 98.68085 & 131.8801 & 0 & 532 \\
\hline Conflict Exposure - $50 \mathrm{~km}$ & 8435 & 51.0735 & 76.17543 & 0 & 383 \\
\hline Distance from Goma & 8435 & 906.0282 & 529.551 & 1.32598 & 1881.164 \\
\hline
\end{tabular}

Because reliable micro-level measures of income or consumption expenditures are not available for the DRC, I use the DHS micro-data to create a simple wealth index that is the sum of a variety of binary indicator variables which are each given equal weight. These indicators include information about whether the household has the following:

- Television ownership

- Refrigerator ownership

- Motor transport ownership (i.e., either a car, truck, motorcycle, or scooter)

- Telephone ownership

- Household electricity

- Radio ownership

- Bicycle ownership 
- Cement floor in the household

- Improved water source in the household

- Mobile phone ownership

- Ownership of a grill or heater

- Ownership of chairs

- Ownership of beds

- Ownership of lamps

- Ownership of a stove or cooker

- Ownership of hoes

- Access to a flush toilet or ventilated improved pit latrine

An improved water source is defined here as water that is piped into a dwelling, yard, or plot, water that is accessible through a public tap or standpipe, water that is from a tube well, water that is from a protected well in a dwelling, yard, or plot, water that is from a protected public well, water that is from a protected spring, or the use of bottled water. All other sources of water are coded as a '0' (e.g. unprotected spring water, river water, etc.). ${ }^{19}$

While this wealth index is by no means a perfect indicator of economic development, it does allow for a relatively more tractable interpretation of how conflict exposure influences household-level durable ownership and living conditions than using the DHS wealth index (which is created via principal components analysis). Figure 2.2 presents a graphical depiction of the distribution of household-level 'wealth' across the DRC. In Section 6, I use the DHS wealth index to show that the results are robust to using either measure of the dependent variable. ${ }^{20}$

\footnotetext{
${ }^{19}$ While some argue that a principal components analysis (PCA) approach is the best method for determining weights for the components of the wealth index (e.g. Filmer and Pritchett, 2001), it is difficult to interpret changes in the PCA wealth index relative to the wealth index constructed here. In the robustness checks below, I use the original DHS wealth index to ensure that the results are not driven by the way my wealth index was created or weighted.

${ }^{20}$ The correlation between my wealth index and the original DHS wealth index is 0.8577 .
} 


\section{Figure 2.2: Distribution (By Quintile) of Cluster-level Average Household- level Wealth (Constructed from DHS Data)}

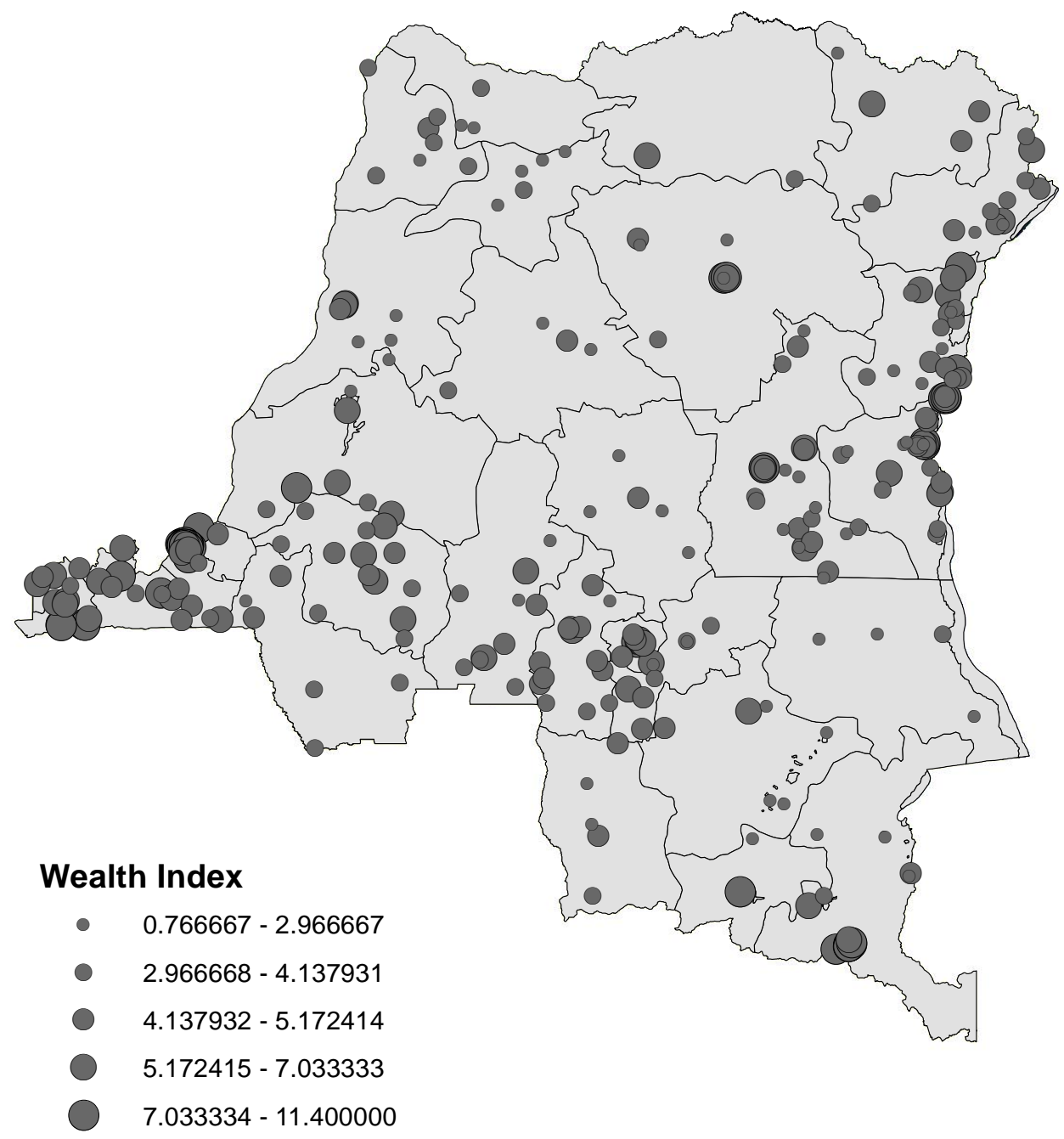

I include a number of control variables in the regressions below, including age of the household head, indicators for whether the household is located in the capital or a large city, a 
small city, or a town, and the altitude of DHS cluster locations. To be sure, some of these control variables may be partially related to conflict exposure. Finding entirely exogenous control variables in this context is a challenging task. Many of the standard control variables used in development studies (e.g., human capital accumulation) will be at least partially endogenous to conflict exposure (see, for instance, Akresh and de Walque, 2008; Chamarbagwala and Moran, 2011).

In some of the regressions below, I include the education of the household head (in years) in order to assess whether controlling for this variable influences the results. Arguably, education (in years) is a good proxy for the historical distribution of expenditures on public goods and public services (like education) by the state and non-state actors (e.g., churches or NGOs). This variable may therefore capture some of the regional factors that influence household-level durable ownership and living conditions, therefore decreasing the likelihood of biasing the instrumental variable estimates.

\subsection{Identification Strategy}

Because of the potential for selection bias, estimates from ordinary least squares regressions will likely be biased and inconsistent. To illustrate why this may be the case, consider that rebel groups, local militias, and Congolese troops seek political control over different areas. Rebel groups would prospectively be attracted to (a) centers of political power (e.g. provincial/national capitals), (b) natural, mineral, and land resources, or (c) areas where the durables of the local populations easily appropriated. Provincial capitals have larger markets, public goods and services (e.g. schools, hospitals, sanitation, piped water, etc.) and therefore likely improved measures of durable ownership and living conditions. At the household level as well, relatively 
wealthier households will attract attacks from rebel groups for the purposes of durable appropriation. ${ }^{21}$ Expropriation appears to be almost systematic, with "nearly all soldiers thus extort[ing] the local residents, stealing all kinds of valuables, such as money or mobile phones in urban areas and harvests or cattle in rural areas. Along with stealing, they often beat, jailed, raped, tortured, or killed those who refused to comply" (Autesserre, 2010: 56). In this sense, there will be a significant degree of reverse causation and a non-random assignment of conflict events when assessing the relationship between wealth and conflict exposure.

I report the estimates from baseline OLS regressions in Table 2.2. The estimates suggest that there is a positive and statistically significant relationship between wealth and conflict exposure. Yet, as suggested above, the OLS estimates are likely biased and inconsistent. This is further confirmed by a Hausman test for the baseline specification (Table 2.3, first model) $\left(\chi^{2}=570.03, \operatorname{Prob}>\chi^{2}=0.000\right)$.

\footnotetext{
${ }^{21}$ As described by Autesserre (2010: 55), "each armed group used violence to deter villagers from supporting some other faction, or to punish them for having done so...all soldiers, in all components, preyed on local villages to make up for receiving little or no income."
} 
Table 2.2: Ordinary Least Squares Regression Results

\begin{tabular}{|c|c|c|c|c|c|c|}
\hline & $(1)$ & (2) & (3) & (4) & (5) & (6) \\
\hline Conflict & $\begin{array}{l}0.00090^{* *} \\
(0.00035)\end{array}$ & $\begin{array}{c}0.00247^{* * *} \\
(0.00065)\end{array}$ & $\begin{array}{l}0.00595^{* * *} \\
(0.00125)\end{array}$ & $\begin{array}{c}0.00125^{* * *} \\
(0.00032)\end{array}$ & $\begin{array}{c}0.00314^{* * *} \\
(0.00057)\end{array}$ & $\begin{array}{c}0.00667^{* * *} \\
(0.00109)\end{array}$ \\
\hline HH Members & $\begin{array}{c}0.14149^{* * *} \\
(0.01157)\end{array}$ & $\begin{array}{c}0.14003^{* * *} \\
(0.01138)\end{array}$ & $\begin{array}{c}0.13744^{* * *} \\
(0.01116)\end{array}$ & $\begin{array}{c}0.11664^{* * *} \\
(0.01051)\end{array}$ & $\begin{array}{c}0.11472^{* * *} \\
(0.01035)\end{array}$ & $\begin{array}{c}0.11253^{* * *} \\
(0.01030)\end{array}$ \\
\hline Capital or Large City & $\begin{array}{c}4.11022^{* * *} \\
(0.23477)\end{array}$ & $\begin{array}{l}3.97926^{* * *} \\
(0.23160)\end{array}$ & $\begin{array}{c}3.71392^{* * *} \\
(0.23928)\end{array}$ & $\begin{array}{c}3.27473^{* * *} \\
(0.21225)\end{array}$ & $\begin{array}{c}3.10524^{* * *} \\
(0.20832)\end{array}$ & $\begin{array}{c}2.84368^{* * *} \\
(0.21400)\end{array}$ \\
\hline Small City & $\begin{array}{l}3.71381^{* * *} \\
(0.80835)\end{array}$ & $\begin{array}{c}3.71121^{* * *} \\
(0.80886)\end{array}$ & $\begin{array}{l}3.71308^{* * *} \\
(0.80363)\end{array}$ & $\begin{array}{l}3.18176^{* * *} \\
(0.67663)\end{array}$ & $\begin{array}{c}3.15017^{* * *} \\
(0.67479)\end{array}$ & $\begin{array}{c}3.12971^{* * *} \\
(0.66981)\end{array}$ \\
\hline Town & $\begin{array}{l}1.93346^{* * * *} \\
(0.24578)\end{array}$ & $\begin{array}{l}1.96764^{* * *} \\
(0.24629)\end{array}$ & $\begin{array}{l}1.93023^{* * * *} \\
(0.24837)\end{array}$ & $\begin{array}{l}1.49202^{* * * *} \\
(0.22966)\end{array}$ & $\begin{array}{l}1.52718^{* * *} \\
(0.23090)\end{array}$ & $\begin{array}{l}1.47954^{* * *} \\
(0.23458)\end{array}$ \\
\hline Altitude (log) & $\begin{array}{c}-0.40726^{* * * *} \\
(0.13895)\end{array}$ & $\begin{array}{c}-0.41235^{* * *} \\
(0.13517)\end{array}$ & $\begin{array}{c}-0.35701^{* * * *} \\
(0.12755)\end{array}$ & $\begin{array}{c}-0.34545^{* * *} \\
(0.13017)\end{array}$ & $\begin{array}{c}-0.33346^{* * * *} \\
(0.12468)\end{array}$ & $\begin{array}{c}-0.24915^{* * *} \\
(0.11790)\end{array}$ \\
\hline $\begin{array}{l}\text { Fraction of } \mathrm{HH} \\
\text { Under } 5\end{array}$ & $\begin{array}{c}-1.24682^{* * *} \\
(0.17352)\end{array}$ & $\begin{array}{c}-1.21971^{* * *} \\
(0.17044)\end{array}$ & $\begin{array}{c}-1.16433^{* * * *} \\
(0.16963)\end{array}$ & $\begin{array}{c}-1.03280^{* * * *} \\
(0.16112)\end{array}$ & $\begin{array}{c}-1.00246^{* * * *} \\
(0.15819)\end{array}$ & $\begin{array}{c}-0.95462^{* * * *} \\
(0.15926)\end{array}$ \\
\hline Male $\mathrm{HHH}$ & $\begin{array}{c}0.71364^{* * *} \\
(0.06745)\end{array}$ & $\begin{array}{c}0.71933^{* * *} \\
(0.06747)\end{array}$ & $\begin{array}{c}0.71751^{* * *} \\
(0.06694)\end{array}$ & $\begin{array}{c}0.06828 \\
(0.07782)\end{array}$ & $\begin{array}{c}0.07147 \\
(0.07769)\end{array}$ & $\begin{array}{c}0.07814 \\
(0.07682)\end{array}$ \\
\hline Age of $\mathrm{HHH}$ & $\begin{array}{c}0.00051 \\
(0.00219)\end{array}$ & $\begin{array}{c}0.00066 \\
(0.00216)\end{array}$ & $\begin{array}{c}0.00029 \\
(0.00216)\end{array}$ & $\begin{array}{c}0.01437^{* * * *} \\
(0.00228)\end{array}$ & $\begin{array}{c}0.01455^{* * *} \\
(0.00226)\end{array}$ & $\begin{array}{c}0.01379^{* * *} \\
(0.00228)\end{array}$ \\
\hline Education of $\mathrm{HHH}$ & & & & $\begin{array}{c}0.18442^{* * *} \\
(0.01089)\end{array}$ & $\begin{array}{c}0.18547^{* * *} \\
(0.01087)\end{array}$ & $\begin{array}{c}0.18258^{* * *} \\
(0.01080)\end{array}$ \\
\hline $\begin{array}{l}\text { Distance Radius } \\
\text { Households }\end{array}$ & $\begin{array}{c}200 \mathrm{~km} \\
8435\end{array}$ & $\begin{array}{c}100 \mathrm{~km} \\
8435\end{array}$ & $\begin{array}{l}50 \mathrm{~km} \\
8435\end{array}$ & $\begin{array}{c}200 \mathrm{~km} \\
8435\end{array}$ & $\begin{array}{c}100 \mathrm{~km} \\
8435\end{array}$ & $\begin{array}{c}50 \mathrm{~km} \\
8435\end{array}$ \\
\hline
\end{tabular}

Clustered standard errors (clustered at the DHS cluster location) in parentheses. Statistical significance is indicated as follows: ${ }^{*} p<0.10,{ }^{* *} p<0.05,{ }^{* * *} p<0.01$. The dependent variable is individual-level wealth. Specifications (1) and (3) use a measure of conflict exposure that counts all conflict events that have taken place within a $200 \mathrm{~km}$ radius. Specifications (2) and (4) are for $100 \mathrm{~km}$ radii and Specifications (3) and (6) $50 \mathrm{~km}$ radii, respectively. A constant term is included in each specification but is not reported.

Because conflict events were not randomly assigned across the country, I use the variation in distances between DHS cluster locations and Goma, a city along the DRC-Rwanda border, as an instrument for conflict exposure. The provinces near Goma have experienced a disproportionate amount of conflict events and this instrumental variables strategy is intended to account for the distribution of conflict events across the country. ${ }^{22}$ One could interpret this

${ }^{22}$ The use of a distance-based instrument has precedent in the empirical literature on conflict and development. Akresh and de Walque (2010) exploit the variation in distances between Ugandan provincial capitals and the Uganda-Rwanda border as an instrument for war intensity in the context of the Rwandan genocide. I employ a 
clustering of conflict events along the DRC-Rwanda border as being a result of the 'spillover' of the Rwandan genocide in addition to localized disputes. Another plausible interpretation is that this relates to being far in distance from the capital, Kinshasa, though so too are many other regions of the country (e.g., Lubumbashi). More credible is the idea that the Congolese government does not exercise much control over the eastern and northeastern provinces of the country as a result of state weakness and the strength of rebel groups maintaining territorial control in these provinces.

A good instrumental variable is exogenous, is highly correlated with the endogenous variable of interest, and is uncorrelated with other unobservable influences of the dependent variable. With high distance radii $(200 \mathrm{~km})$, the correlation between conflict exposure and the distance instrument is high $(-0.6424$ for the correlation between distances from Goma and conflict exposure; -0.6830 when only those recent conflict events in the eastern provinces are considered), though for low distance radii (e.g. $50 \mathrm{~km})$, the correlation is not as strong (-0.1721), mainly because the conflict events taking place near Kinshasa are, in effect, weighted more heavily (see the scatterplots in Figure 2.3 for a graphical depiction).

similar strategy, endogenously determining the intensity of conflict exposure across the country on the basis of distances between DHS cluster locations and Goma. 
Figure 2.3: Scatterplots of Conflict Exposure (ACLED) and Distance from Goma

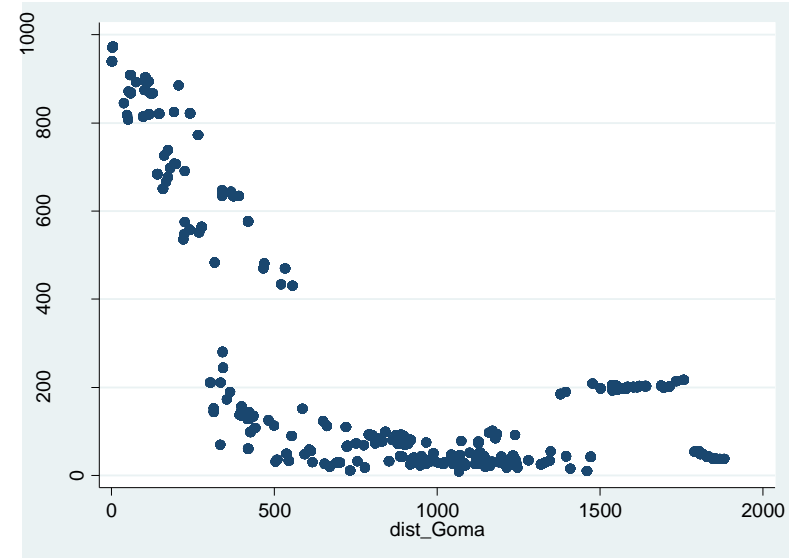

Conflict exposure within $200 \mathrm{~km}$

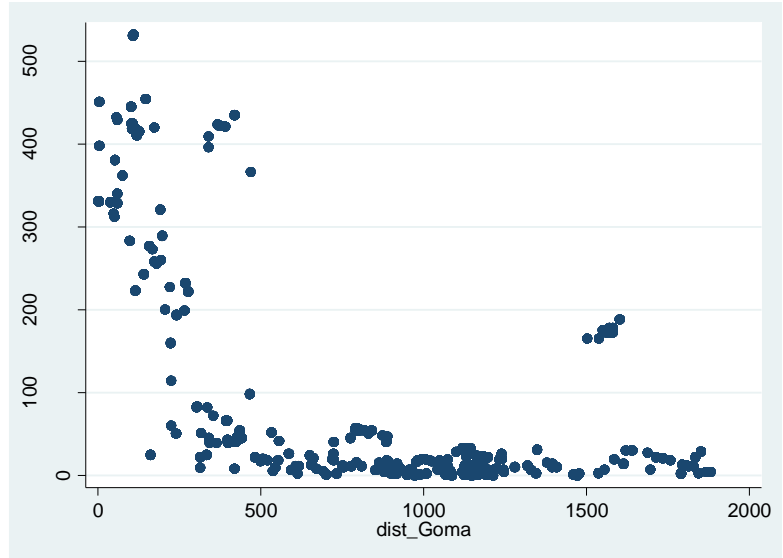

Conflict Exposure within $100 \mathrm{~km}$

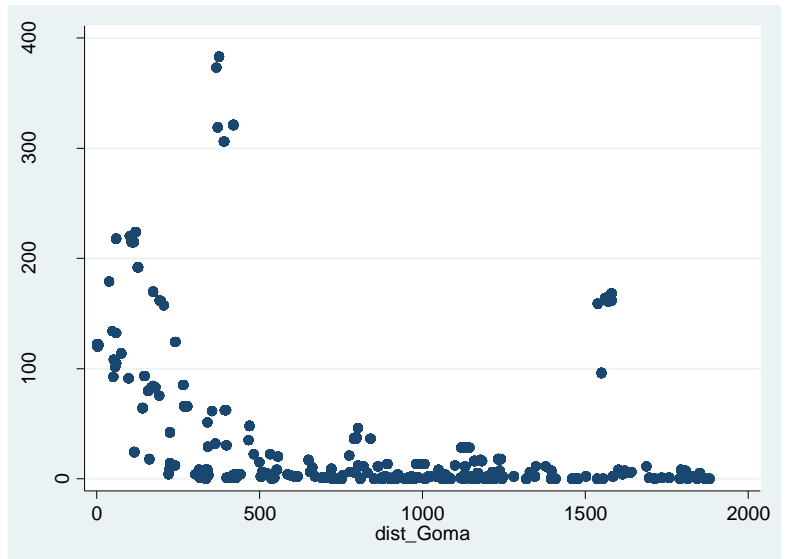

Conflict Exposure within $50 \mathrm{~km}$
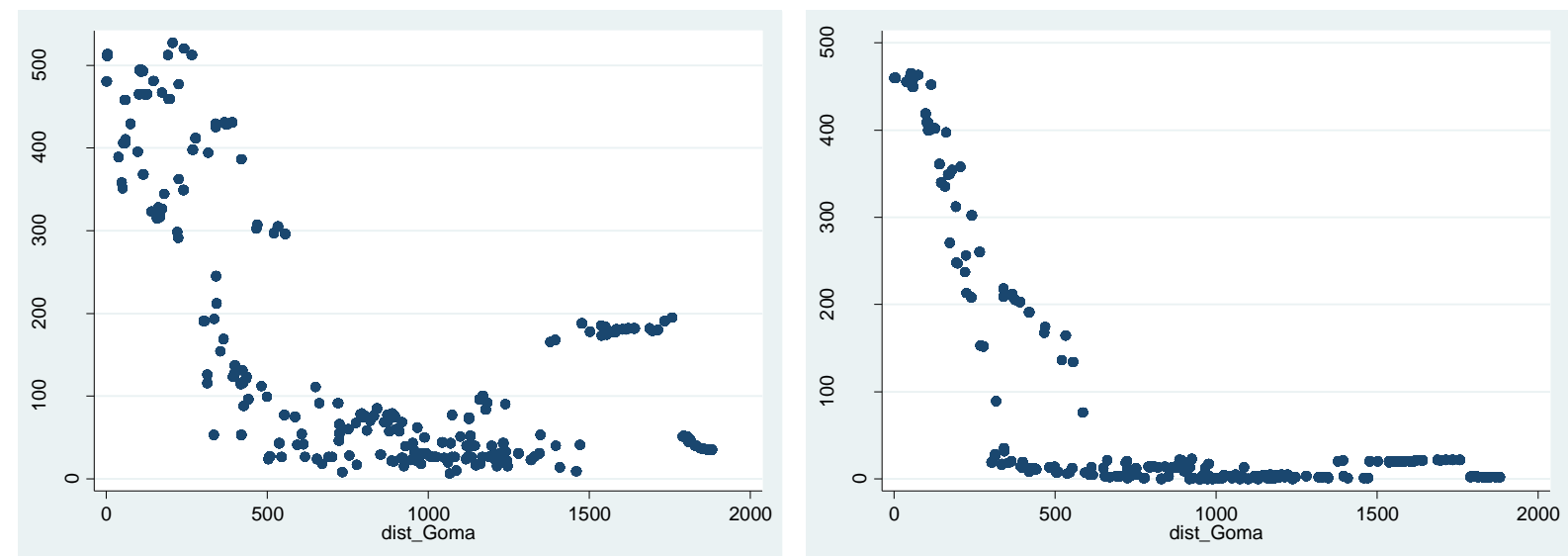

Conflict exposure within $200 \mathrm{~km}$, first and second civil wars and conflicts in the eastern provinces, respectively 
There are plausible scenarios in which the exclusion restriction may not be met (e.g. differential provision of public goods across geographic areas), though this is difficult to assess in practice. The identification strategy employed in this paper implicitly assumes that the distance measures only influence household-level wealth through their effects on conflict exposure, conditional on the set of included covariates (e.g. rural/urban differences, age, etc.). One way I control for the historical distribution of expenditures on public goods (e.g. public spending on education) is to include education (in years) as an explanatory variable. As stated above, I do not interpret the coefficient estimate on this variable as representing something causal - it is merely a control variable. But this control variable should help to capture the effects of the distance from the border or other previous policy factors so that the interpretation of the coefficient on conflict exposure can be seen as representing something causal. Because the equation below is exactly identified, I do not conduct a Sargan-Hansen test. I report the first stage results for the Angrist-Pischke multivariate F test of excluded instruments in the results tables below.

For the baseline IV specification, I model differences in micro-level wealth across the country as a function of conflict exposure, while instrumenting for conflict exposure using the distances between DHS clusters and Goma:

$$
\begin{aligned}
& \text { exposure }_{i}=\alpha_{1}+\mathbf{x}_{\mathrm{i}}^{\prime} \beta+\delta \text { dist }_{\mathrm{i}}+\varepsilon_{\mathrm{i} 1} \\
& \text { wealth }_{\mathrm{i}}=\alpha_{2}+\mathbf{x}_{\mathrm{i}}^{\prime} \gamma+\eta \text { exposure }_{\mathrm{i}}+\varepsilon_{\mathrm{i} 2}
\end{aligned}
$$

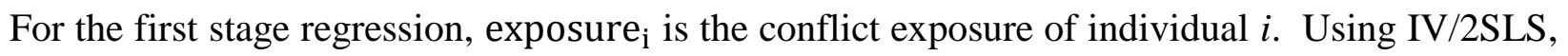
this dependent variable is regressed on a vector of individual-level covariates $\left(\mathbf{x}_{\mathrm{i}}\right)$ and the distances between DHS clusters and Goma ( dist $\left._{\mathrm{i}}\right)$, in addition to the individual-level error term, 
$\varepsilon_{\mathrm{i} 1} \cdot{ }^{23}$ For the second-stage regression, wealth $\mathrm{i}$ is regressed on individual level covariates $\left(\mathbf{x}_{\mathrm{i}}\right)$ and the measure of exposure, (exposure ${ }_{\mathrm{i}}$ ) as well as the individual-level error term, $\varepsilon_{\mathrm{i} 2}$. IV regressions are conducted using the 'ivreg2' package in Stata (Baum et al., 2010).

There are likely to be unobservable factors that influence micro-level measures of durable ownership and living conditions that are systematically correlated over space. I therefore estimate the IV regression models using cluster-robust variance estimates. ${ }^{24}$ This strategy results in smaller, more conservative t-statistics, but the standard errors are more robust to potential heteroskedasticity and intra-cluster correlation.

\subsubsection{Potential Limitations}

It is plausible that the impact of violent conflict on economic development may be underestimated. While the DHS is a nationally representative survey, the conflicts in the DRC particularly the second civil war-led to targeted attacks on the civilian population, significant loss of life, and widespread internal displacement. Those who have been most exposed to conflict events over time (i.e. those killed directly or indirectly by conflict, those whose homes were completely looted or destroyed, etc.) will not be represented in the DHS sample, particularly considering that the civil wars resulted in the internal displacement of nearly 2 million Congolese citizens (Autesserre, 2010: 5). There may be mortality-related selection bias and this would lead to bias in the estimated coefficient for 'conflict exposure.' It is also important to bear in mind that the conflict events in the DRC may have increased investment risk or the capacity of the state at the country level such that the effects of conflict exposure may not appear to have any effect on micro-level wealth.

\footnotetext{
${ }^{23}$ All first stage results are available from the author upon request.

${ }^{24}$ The formula for the cluster-robust error variance-covariance estimator can be found in Baum (2006: 139).
} 
One potential limitation of using conflict exposure as a measure of the damage caused by conflict events in the DRC is that the history of conflict events in particular areas is only a proxy for the magnitude of damage to physical capital, infrastructure, and individual dwellings in particular regions. A single conflict event may actually have as similar an impact as two conflict events (in terms of deaths, damage to capital or infrastructure, etc.), for instance, but they are assigned different values in the conflict exposure data. Also, one single conflict event in an area may have led to the death of a family member, displacement and public health issues in a small community, or severe psychological trauma, which are incidents that may seriously hamper durable ownership and living conditions. This may prove to be a source of measurement error in the instrumental variables regressions. Nevertheless, the ACLED data can serve as a good proxy for the overall level of violent political activity, local insecurity, weak rule of law, and other political economy factors that systematically affect economic development.

Since economies can bounce back in the wake of conflict (Davis and Weinstein, 2002; Brakman et al., 2004; Miguel and Roland, 2011), finding evidence of no effect of conflict on development is not implausible. As noted by Autesserre (2010: 4), after the two civil wars, political transition, and international diplomatic and humanitarian interventions, many families returned home, rebuilt their homes, and engaged in local markets. However, conflict events were still taking place in the eastern and northeastern provinces at the time of writing, which suggests that there will likely be evidence of a negative effect when conflict events in the eastern provinces are considered separately (below).

With the available data, I cannot be certain whether household-level economic outcomes are not caused by conflict exposure in another region or even another country. High degrees of conflict exposure elsewhere may have led to relocations to areas in the DRC that are relatively 
safer, a factor that would be associated with less precision in estimating the effects of conflict exposure on economic outcomes. Yet, if anything, this would produce a positive bias if the relationship between conflict exposure and economic outcomes were negative, as these individuals would likely self-select into areas that were relatively more secure. Transient citizens or visitors will have been exposed to less conflict exposure when surveyed, but will also have worse economic outcomes due to relocation. For these individuals, this would imply that the relationship between durable ownership and living standards is not as negatively related to my measure of conflict exposure, putting upward bias on the estimates.

\subsection{Results}

The results from the baseline instrumental variable regressions are presented in Table 2.3. In the first column, a measure of conflict exposure that accounts for conflict events that have taken place within a $200 \mathrm{~km}$ distance radius of DHS cluster locations is used as the key explanatory variable of interest. The estimates suggest that the mean cluster-level exposure to conflict events within $200 \mathrm{~km}$ (about 231 conflict events) is associated with a 14.7 percent decrease in the wealth index. ${ }^{25}$ This negative coefficient estimate contrasts sharply with OLS estimates that do not account for the potential for reverse causation and a non-random assignment of conflict events. Similarly, the average cluster-level exposure to conflict events within $100 \mathrm{~km}$ (about 100 conflict events) is associated with a 16.7 percent decrease in the wealth index. Lastly, for the mean cluster-level exposure to conflict events within $50 \mathrm{~km}$ (about 52 conflict events) is associated with a 27.9 percent decrease in the wealth index. For the first-stage regression results for the first specification (Column 1), the Angrist-Pischke multivariate $\mathrm{F}$ test of excluded

\footnotetext{
${ }^{25}$ The wealth index has a mean of 5.021816 .
} 
instruments is 97.77 and I can therefore reject the null hypothesis of weak identification given

Stock-Yogo critical values (16.38 for $10 \%$ maximal IV size).

Table 2.3: Instrumental Variable Regression Results

\begin{tabular}{|c|c|c|c|c|c|c|}
\hline & (1) & (2) & (3) & (4) & (5) & (6) \\
\hline Conflict & $\begin{array}{l}-0.00319^{* * * *} \\
(0.00067)\end{array}$ & $\begin{array}{l}-0.00839^{* * *} \\
(0.00186)\end{array}$ & $\begin{array}{c}-0.02699^{* * * *} \\
(0.00783)\end{array}$ & $\begin{array}{c}-0.00248^{* * * *} \\
(0.00060)\end{array}$ & $\begin{array}{l}-0.00656^{* * *} \\
(0.00167)\end{array}$ & $\begin{array}{l}-0.02124^{* * *} \\
(0.00675)\end{array}$ \\
\hline HH Members & $\begin{array}{c}0.14444^{* * *} \\
(0.01386)\end{array}$ & $\begin{array}{c}0.14931^{* * *} \\
(0.01493)\end{array}$ & $\begin{array}{c}0.16344^{* * *} \\
(0.02055)\end{array}$ & $\begin{array}{c}0.12334^{* * * *} \\
(0.01218)\end{array}$ & $\begin{array}{c}0.12761^{* * *} \\
(0.01293)\end{array}$ & $\begin{array}{l}0.13916^{* * * *} \\
(0.01691)\end{array}$ \\
\hline Capital or Large City & $\begin{array}{l}4.19459^{* * * *} \\
(0.26929)\end{array}$ & $\begin{array}{c}4.63727^{* * * *} \\
(0.32213)\end{array}$ & $\begin{array}{l}6.01144^{* * * *} \\
(0.73527)\end{array}$ & $\begin{array}{l}3.48676^{* * * *} \\
(0.24310)\end{array}$ & $\begin{array}{l}3.84819^{* * * *} \\
(0.29192)\end{array}$ & $\begin{array}{l}4.94547^{* * *} \\
(0.63887)\end{array}$ \\
\hline Small City & $\begin{array}{l}2.75155^{* * * *} \\
(0.75344)\end{array}$ & $\begin{array}{l}2.79034^{* * * *} \\
(0.74814)\end{array}$ & $\begin{array}{l}2.54384^{* * * *} \\
(0.76848)\end{array}$ & $\begin{array}{l}2.40344^{* * * *} \\
(0.64749)\end{array}$ & $\begin{array}{l}2.44117^{* * * *} \\
(0.64684)\end{array}$ & $\begin{array}{l}2.25423^{* * *} \\
(0.66168)\end{array}$ \\
\hline Town & $\begin{array}{l}1.70802^{* * *} \\
(0.31430)\end{array}$ & $\begin{array}{l}1.59880^{* * * *} \\
(0.32918)\end{array}$ & $\begin{array}{l}1.67325^{* * * *} \\
(0.45885)\end{array}$ & $\begin{array}{l}1.36131^{\text {**** }} \\
(0.29213)\end{array}$ & $\begin{array}{l}1.28319^{* * * *} \\
(0.30217)\end{array}$ & $\begin{array}{l}1.34814^{* * * *} \\
(0.39650)\end{array}$ \\
\hline Altitude (log) & $\begin{array}{c}0.28353 \\
(0.18923)\end{array}$ & $\begin{array}{c}0.27935 \\
(0.18198)\end{array}$ & $\begin{array}{c}0.20704 \\
(0.19860)\end{array}$ & $\begin{array}{c}0.26548 \\
(0.17369)\end{array}$ & $\begin{array}{c}0.26259 \\
(0.16888)\end{array}$ & $\begin{array}{c}0.20602 \\
(0.17920)\end{array}$ \\
\hline $\begin{array}{l}\text { Fraction of } \mathrm{HH} \\
\text { Under } 5\end{array}$ & $\begin{array}{c}-1.45415^{* * *} \\
(0.21421)\end{array}$ & $\begin{array}{c}-1.53989^{* * *} \\
(0.23899)\end{array}$ & $\begin{array}{c}-1.87396^{* * *} \\
(0.35008)\end{array}$ & $\begin{array}{c}-1.25407^{* * *} \\
(0.19818)\end{array}$ & $\begin{array}{c}-1.32541^{* * *} \\
(0.21834)\end{array}$ & $\begin{array}{c}-1.59262^{* * * *} \\
(0.30739)\end{array}$ \\
\hline Male HHH & $\begin{array}{l}0.69835^{* * *} \\
(0.07554)\end{array}$ & $\begin{array}{c}0.67948^{* * *} \\
(0.07926)\end{array}$ & $\begin{array}{l}0.67744^{* * *} \\
(0.09646)\end{array}$ & $\begin{array}{l}0.15973^{*} \\
(0.08284)\end{array}$ & $\begin{array}{l}0.15639^{*} \\
(0.08619)\end{array}$ & $\begin{array}{c}0.16532 \\
(0.10431)\end{array}$ \\
\hline Age of $\mathrm{HHH}$ & $\begin{array}{l}-0.00289 \\
(0.00249)\end{array}$ & $\begin{array}{l}-0.00330 \\
(0.00269)\end{array}$ & $\begin{array}{l}-0.00265 \\
(0.00338)\end{array}$ & $\begin{array}{l}0.00906^{* * * *} \\
(0.00244)\end{array}$ & $\begin{array}{c}0.00849^{* * *} \\
(0.00260)\end{array}$ & $\begin{array}{c}0.00876^{* * *} \\
(0.00317)\end{array}$ \\
\hline Education of $\mathrm{HHH}$ & & & & $\begin{array}{c}0.15436^{* * *} \\
(0.01192)\end{array}$ & $\begin{array}{c}0.15109^{* * *} \\
(0.01275)\end{array}$ & $\begin{array}{c}0.14804^{* * *} \\
(0.01622)\end{array}$ \\
\hline Distance Radius & $200 \mathrm{~km}$ & $100 \mathrm{~km}$ & $50 \mathrm{~km}$ & $200 \mathrm{~km}$ & $100 \mathrm{~km}$ & $50 \mathrm{~km}$ \\
\hline Households & 8435 & 8435 & 8435 & 8435 & 8435 & 8435 \\
\hline $\begin{array}{l}\text { F test of excluded } \\
\text { instruments }\end{array}$ & 97.77 & 72.23 & 21.89 & 96.02 & 71.32 & 22.08 \\
\hline $2^{\text {nd }}$ Stage F statistic & 63.59 & 51.72 & 27.87 & 75.94 & 62.51 & 37.18 \\
\hline $\begin{array}{l}\text { Distance from Goma } \\
\left(1^{\text {st }} \text { Stage }\right)\end{array}$ & $\begin{array}{c}-.3342609^{* * * *} \\
(.033805) \\
\end{array}$ & $\begin{array}{l}-.1271951^{* * * *} \\
(.0149666)\end{array}$ & $\begin{array}{l}-.0395232^{* * * *} \\
(.0084476)\end{array}$ & $\begin{array}{l}-.3309605^{* * *} \\
(.0337752)\end{array}$ & $\begin{array}{l}-.1251811^{* * * *} \\
(.0148227)\end{array}$ & $\begin{array}{c}-0386808^{* * * * *} \\
(.0082323)\end{array}$ \\
\hline $\begin{array}{l}\text { Clustered standard error } \\
p<0.10,{ }^{* *} p<0.05,{ }^{* * *} \\
\text { conflict exposure that co } \\
100 \mathrm{~km} \text { radii and Specifi } \\
\text { stage F-test statistic is th }\end{array}$ & ustered at the & IS cluster locat & ) in parenthes & Statistical sig & icance is indica & $\begin{array}{l}\text { d as follows: } \\
\text { se a measure of } \\
\text { and (4) are for } \\
\text { iments. The } 2^{\text {nd }}\end{array}$ \\
\hline
\end{tabular}

As discussed above, the historical distribution of public spending on schools and hospitals across countries may be one of the factors that violate the exclusion restriction of the 
IV approach. In other words, household-level wealth may be related to 'distance from Goma' in other ways than the impact of distance from the DRC-Rwanda border on conflict exposure. Including education (in years) may be one way to control for this confounding influence. When I control for education (in years), the magnitude of the coefficient on conflict exposure is somewhat less. Column 4 of Table 2.3 presents the baseline results where distance from Goma is used as an instrument for the number of conflict events within a $200 \mathrm{~km}$ distance. The coefficient estimate is -0.00248 , which, at a mean level of cluster-level conflict exposure of about 231 results in a reduction of the wealth index by 11.4 percent.

\subsection{Robustness Checks}

\subsubsection{Cluster-level Control Variables}

One of the limitations of the results in Table 2.3 is that there are no cluster-level covariates in the regression models. In this section, I incorporate cluster-level control variables on the locations of mining sites, roads, and airports. By doing so, I am able to test the robustness of the baseline results by controlling for some of the geographical and economic environmental factors that may influence household-level economic conditions, conflict events, or both.

Data on the locations of mining concessions comes from the International Peace Information Service (IPIS) in Antwerp, Belgium. The IPIS has developed a series of maps on the mineral sector of the DRC. For this paper, I use data from their map on mine concessions across the country. ${ }^{26}$ The data are official and public data from the DRC's Cadastre Minier (CaMi). I specifically focus on mining concessions where the companies involved have an exploitation permit, a tailings processing permit, a small-scale mining exploitation permit, or a permanent

\footnotetext{
${ }^{26}$ This map is available at http://www.ipisresearch.be/mine-concessions-drc.php
} 
quarry exploitation authorization. These activities should sufficiently capture mining activity (rather than general exploration permits or applications for exploration and/or exploitation permits). In order to match the mining data with the household-level wealth and conflict data, I only use those mining sites for which the company or corporation had their concession granted to them by the government prior to $2007 .{ }^{27}$ I then calculate the total size of the shapefile areas for the mining sites within specified distances of DHS cluster locations. This calculation will capture the relative magnitude of mining activity within certain vicinities of the DHS clusters.

Information on the locations and lengths of primary and secondary roads comes from the roads shapefile from the African Development Bank Group. ${ }^{28}$ I calculate the total length of roads within specific distances of DHS cluster locations in order to control for road density and other geographic attributes of DHS cluster locations. Information about the location of paved, IATAcoded airports across the country is drawn from Air Broker Center International AB. ${ }^{29}$ Lastly, I use shapefiles on administrative boundaries for the DRC from FAO's Africover. ${ }^{30}$ Summary statistics for all of the variables used in this subsection are presented in Table 2.4.

\footnotetext{
${ }^{27}$ The data on granted mining concessions run from April 1994 to the end of 2006 (for this analysis).

${ }^{28}$ The shapefile is available at http://www.infrastructureafrica.org/documents/type/arcgis-shape-files/congo-dr

${ }^{29}$ I thank Tilman Brück at DIW Berlin for suggesting the use of airports as a control variable. The data on airports across the DRC are available at http://www.aircraft-charter-world.com/airports/africa/congodr.htm. I only geo-code airports that have paved runways and IATA codes. While it would be interesting to geo-code all of the airports that are listed on this site, I could only visually confirm the locations of all of the paved, IATA-coded airports through Google Earth. This provided a natural guideline for which airports to include in the analysis.

${ }^{30}$ The shapefile for administrative boundaries for the DRC is available at http://www.africover.org/.
} 


\section{Table 2.4: Summary Statistics -Variables Used in Section 2.6}

\begin{tabular}{lccccc}
\hline & & & & & \\
& \# Obs. & Mean & Std. Dev. & Min & Max \\
\hline Original DHS Wealth Index & 8435 & 3.189212 & 100165.6 & -107521 & 345191 \\
Wealth Index - Liquid & 8435 & 3.138115 & 1.655543 & 0 & 8 \\
Wealth Index - Illiquid & 8435 & 1.883699 & 1.693944 & 0 & 9 \\
DHS Cluster Latitude & 8435 & -3.689572 & 3.48932 & -11.7621 & 4.640158 \\
DHS Cluster Longitude & 8435 & 22.56878 & 5.36442 & 12.61893 & 30.96663 \\
Airports $(200 \mathrm{~km})$ & 8435 & 1.300771 & .759256 & 0 & 3 \\
Airports $(100 \mathrm{~km})$ & 8435 & .6570243 & .7433821 & 0 & 2 \\
Airports $50 \mathrm{~km})$ & 8435 & .4713693 & .6978944 & 0 & 2 \\
Mining Sites $(200 \mathrm{~km})$ & 8435 & .1803309 & .234341 & 0 & .9290972 \\
Mining Sites $(100 \mathrm{~km})$ & 8435 & .0602926 & .1138374 & 0 & .5072917 \\
Mining Sites $(50 \mathrm{~km})$ & 8435 & .0163952 & .0365602 & 0 & .2383333 \\
Total Road Length $(200 \mathrm{~km})$ & 8435 & 2138.083 & 574.4519 & 786.4307 & 3586.059 \\
Total Road Length $(100 \mathrm{~km})$ & 8435 & 763.8113 & 231.5467 & 0 & 1535.314 \\
Total Road Length $(50 \mathrm{~km})$ & 8435 & 336.9962 & 156.438 & 0 & 734.8842 \\
Conflict - GED $(200 \mathrm{~km})$ & 8435 & 77.65975 & 110.132 & 0 & 406 \\
Conflict - GED $(100 \mathrm{~km})$ & 8435 & 33.98814 & 53.78902 & 0 & 208 \\
Conflict - GED $(50 \mathrm{~km})$ & 8435 & 16.51879 & 24.80985 & 0 & 120 \\
Conflict - Wars/Civ $(200 \mathrm{~km})$ & 8435 & 116.6333 & 129.0029 & 5 & 470 \\
Conflict - Wars/Civ $(100 \mathrm{~km})$ & 8435 & 47.59312 & 63.11589 & 0 & 292 \\
Conflict - Wars/Civ $(50 \mathrm{~km})$ & 8435 & 23.34215 & 39.01607 & 0 & 253 \\
Conflict - East/Civ $(200 \mathrm{~km})$ & 8435 & 64.76574 & 121.7141 & 0 & 411 \\
Conflict - East/Civ $(100 \mathrm{~km})$ & 8435 & 28.28477 & 60.14331 & 0 & 255 \\
Conflict - East/Civ $(50 \mathrm{~km})$ & 8435 & 12.46663 & 26.89498 & 0 & 124 \\
Distance from Kinshasa & 8435 & 911.8457 & 572.2951 & .5452207 & 1903.349 \\
\hline
\end{tabular}

Using these three cluster-level control variables, I find very similar results regarding the impact of conflict on household-level durable ownership and living conditions (Table 2.5). The coefficient estimates are only slightly smaller while they remain highly statistically significant. I find strong evidence that airports are positively associated with household-level durable ownership and living conditions, though the direction of causation in this situation is uncertain since the locations of airports may reflect population density and the clustering of wealth, rather than causing greater access to international markets or trade networks. Nevertheless, the locations of airports should serve as a decent proxy for historical pockets of wealth across the country, which may make inference of the impact of conflict on development more precise. Further, the locations of airports may also influence the location of conflict events, as discussed in Subsection 2.2. Indeed, there is a strong conditional correlation between the number of 
airports that are within cluster radii and the number of conflict events to which a cluster has been exposed.

\subsubsection{Different Measure of Household-level Wealth}

Another potential critique of the results in Table 2.3 is that household-level wealth may be poorly measured by the simple index of durable ownership and living conditions. In this subsection, I therefore consider using the original DHS wealth index as the dependent variable. This strategy allows for the use of a more comprehensive measure of household-level wealth but has the disadvantage of making the coefficient interpretation somewhat more challenging. Figure 2.4 presents the distribution (by quintile) of household-level wealth, as measured by the original DHS wealth index.

Using the original DHS wealth index as the dependent variable, I find very similar results (Presented in Table 2.6). Conflict exposure has a negative and significant impact on householdlevel wealth and the magnitude of this effect is comparable to the estimates in the earlier regression results. One of the initial reasons for using this index was its difficulty in interpreting the estimated coefficients of the explanatory and control variables. Nonetheless, the relative magnitudes, absolute magnitudes, and signs of the estimated coefficients - as well as their statistical significance - are remarkably similar to the prior results. These results also hold when using the latitude coordinate and longitude coordinate of DHS cluster locations as separate instruments for conflict exposure (various distance radii). 


\section{Table 2.5: Instrumental Variable Regression Results - Inclusion of Cluster-level Control Variables}

\begin{tabular}{|c|c|c|c|c|c|c|}
\hline & $(1)$ & (2) & (3) & (4) & (5) & (6) \\
\hline Conflict & $\begin{array}{c}-0.00327^{* * * *} \\
(0.00083)\end{array}$ & $\begin{array}{c}-0.00748^{* * * *} \\
(0.00177)\end{array}$ & $\begin{array}{l}-0.02165^{* * * *} \\
(0.00589)\end{array}$ & $\begin{array}{c}-0.00239^{\text {***** }} \\
(0.00076)\end{array}$ & $\begin{array}{c}-0.00575^{* * *} \\
(0.00164)\end{array}$ & $\begin{array}{l}-0.01701^{\text {***** }} \\
(0.00540)\end{array}$ \\
\hline HH Members & $\begin{array}{c}0.14954^{* * *} \\
(0.01339)\end{array}$ & $\begin{array}{c}0.13795^{* * *} \\
(0.01376)\end{array}$ & $\begin{array}{c}0.13271^{* * *} \\
(0.01572)\end{array}$ & $\begin{array}{c}0.12738^{* * *} \\
(0.01168)\end{array}$ & $\begin{array}{c}0.11841^{* * *} \\
(0.01210)\end{array}$ & $\begin{array}{c}0.11437^{* * *} \\
(0.01370)\end{array}$ \\
\hline Capital or Large City & $\begin{array}{c}3.55043^{* * * *} \\
(0.26294)\end{array}$ & $\begin{array}{c}3.17756^{* * *} \\
(0.37564)\end{array}$ & $\begin{array}{l}1.52753^{* *} \\
(0.66782)\end{array}$ & $\begin{array}{c}2.88101^{* * * *} \\
(0.23455)\end{array}$ & $\begin{array}{c}2.63048^{* * * *} \\
(0.33709)\end{array}$ & $\begin{array}{l}1.23631^{* * *} \\
(0.57677)\end{array}$ \\
\hline Small City & $\begin{array}{c}2.57419^{* * *} \\
(0.89098)\end{array}$ & $\begin{array}{l}2.85622^{* * *} \\
(0.74745)\end{array}$ & $\begin{array}{l}2.27233^{* * *} \\
(0.77289)\end{array}$ & $\begin{array}{l}2.22814^{* * *} \\
(0.75206)\end{array}$ & $\begin{array}{c}2.51433^{* * *} \\
(0.65823)\end{array}$ & $\begin{array}{l}2.03346^{* * *} \\
(0.69370)\end{array}$ \\
\hline Town & $\begin{array}{l}1.67407^{* * * *} \\
(0.27645)\end{array}$ & $\begin{array}{l}1.68722^{* * * *} \\
(0.31573)\end{array}$ & $\begin{array}{l}1.81935^{* * *} \\
(0.38279)\end{array}$ & $\begin{array}{l}1.31482^{* * *} \\
(0.25413)\end{array}$ & $\begin{array}{l}1.36806^{* * *} \\
(0.29074)\end{array}$ & $\begin{array}{l}1.48453^{* * * *} \\
(0.33886)\end{array}$ \\
\hline Altitude (log) & $\begin{array}{c}0.29078^{*} \\
(0.16984)\end{array}$ & $\begin{array}{c}0.31722^{*} \\
(0.18509)\end{array}$ & $\begin{array}{l}0.45236^{* * *} \\
(0.21518)\end{array}$ & $\begin{array}{c}0.27319^{*} \\
(0.15418)\end{array}$ & $\begin{array}{c}0.29628^{*} \\
(0.17375)\end{array}$ & $\begin{array}{l}0.41592^{* *} \\
(0.19775)\end{array}$ \\
\hline $\begin{array}{l}\text { Fraction of HH } \\
\text { Under } 5\end{array}$ & $\begin{array}{c}-1.30852^{* * *} \\
(0.19206)\end{array}$ & $\begin{array}{c}-1.30840^{* * *} \\
(0.20265)\end{array}$ & $\begin{array}{c}-1.33813^{* * *} \\
(0.22213)\end{array}$ & $\begin{array}{c}-1.09784^{* * *} \\
(0.17610)\end{array}$ & $\begin{array}{c}-1.12754^{* * *} \\
(0.18953)\end{array}$ & $\begin{array}{c}-1.15591^{* * *} \\
(0.20337)\end{array}$ \\
\hline Male HHH & $\begin{array}{c}0.68827^{* * *} \\
(0.07321)\end{array}$ & $\begin{array}{c}0.70330^{* * *} \\
(0.07382)\end{array}$ & $\begin{array}{c}0.72377^{* * * *} \\
(0.07654)\end{array}$ & $\begin{array}{c}0.11860 \\
(0.07965)\end{array}$ & $\begin{array}{l}0.18757^{* *} \\
(0.08237)\end{array}$ & $\begin{array}{l}0.22677^{* * *} \\
(0.08751)\end{array}$ \\
\hline Age of $\mathrm{HHH}$ & $\begin{array}{l}-0.00210 \\
(0.00224)\end{array}$ & $\begin{array}{l}-0.00293 \\
(0.00223)\end{array}$ & $\begin{array}{l}-0.00222 \\
(0.00268)\end{array}$ & $\begin{array}{l}0.01056^{* * *} \\
(0.00223)\end{array}$ & $\begin{array}{c}0.00859^{* * *} \\
(0.00222)\end{array}$ & $\begin{array}{l}0.00863^{* * *} \\
(0.00254)\end{array}$ \\
\hline Airports & $\begin{array}{c}0.94749^{* * * *} \\
(0.16933)\end{array}$ & $\begin{array}{l}1.32818^{* * *} \\
(0.28280)\end{array}$ & $\begin{array}{l}3.35875^{* * *} \\
(0.69671)\end{array}$ & $\begin{array}{c}0.81884^{* * *} \\
(0.15124)\end{array}$ & $\begin{array}{l}1.12350^{* * *} \\
(0.25798)\end{array}$ & $\begin{array}{c}2.81113^{* * *} \\
(0.63489)\end{array}$ \\
\hline Mining Sites & $\begin{array}{c}0.52967 \\
(0.66158)\end{array}$ & $\begin{array}{c}-2.37437^{* * *} \\
(0.86309)\end{array}$ & $\begin{array}{l}-5.20907 \\
(3.56405)\end{array}$ & $\begin{array}{c}0.34647 \\
(0.60393)\end{array}$ & $\begin{array}{c}-2.15177^{* * *} \\
(0.76142)\end{array}$ & $\begin{array}{l}-4.69903 \\
(3.13621)\end{array}$ \\
\hline Road Length & $\begin{array}{c}-0.00111^{* * *} \\
(0.00027)\end{array}$ & $\begin{array}{c}0.00002 \\
(0.00047)\end{array}$ & $\begin{array}{l}-0.00002 \\
(0.00078)\end{array}$ & $\begin{array}{c}-0.00102^{* * *} \\
(0.00025)\end{array}$ & $\begin{array}{c}0.00001 \\
(0.00043)\end{array}$ & $\begin{array}{c}0.00008 \\
(0.00070)\end{array}$ \\
\hline Education of $\mathrm{HHH}$ & & & & $\begin{array}{l}0.16325^{* * *} \\
(0.01149)\end{array}$ & $\begin{array}{c}0.14781^{* * *} \\
(0.01200)\end{array}$ & $\begin{array}{l}0.14158^{* * *} \\
(0.01337)\end{array}$ \\
\hline Distance Radius & $200 \mathrm{~km}$ & $100 \mathrm{~km}$ & $50 \mathrm{~km}$ & $200 \mathrm{~km}$ & $100 \mathrm{~km}$ & $50 \mathrm{~km}$ \\
\hline Households & 8435 & 8435 & 8435 & 8435 & 8435 & 8435 \\
\hline $\begin{array}{l}\text { F test of excluded } \\
\text { instruments }\end{array}$ & 94.07 & 87.51 & 38.68 & 93.05 & 85.39 & 37.35 \\
\hline 2nd Stage F statistic & 60.11 & 55.31 & 52.63 & 75.01 & 68.43 & 68.60 \\
\hline $\begin{array}{l}\text { Distance from Goma } \\
\text { (1st Stage) }\end{array}$ & $\begin{array}{c}-.3045628^{* * * *} \\
(.0314015)\end{array}$ & $\begin{array}{c}-.1318439^{* * *} \\
(.0140939)\end{array}$ & $\begin{array}{l}-.045394^{* * * *} \\
(.0072993)\end{array}$ & $\begin{array}{l}-.30364^{* * *} \\
(.0314783)\end{array}$ & $\begin{array}{c}-.1291978^{* * *} \\
(.0139811)\end{array}$ & $\begin{array}{l}-.0440591^{* * * *} \\
(.0072088)\end{array}$ \\
\hline \multicolumn{7}{|c|}{$\begin{array}{l}\text { Clustered standard errors (clustered at the DHS cluster location) in parentheses. Statistical significance is indicated as follows: } \\
p<0.10,{ }^{* *} p<0.05,{ }^{* * * *} p<0.01 \text {. The dependent variable is individual-level wealth. Specifications (1) and (3) use a measure of } \\
\text { conflict exposure that counts all conflict events that have taken place within a } 200 \mathrm{~km} \text { radius. Specifications (2) and (4) are for } \\
100 \mathrm{~km} \text { radii and Specifications (3) and (6) } 50 \mathrm{~km} \text { radii. The Hansen J over identification statistic reported above is the Chi- } \\
\text { square p-value for the over identification statistic. A constant term is included in each specification but is not reported. }\end{array}$} \\
\hline
\end{tabular}


Figure 2.4: Distribution (By Quintile) of Cluster-level Average Householdlevel Wealth (Using the Original DHS Wealth Index)

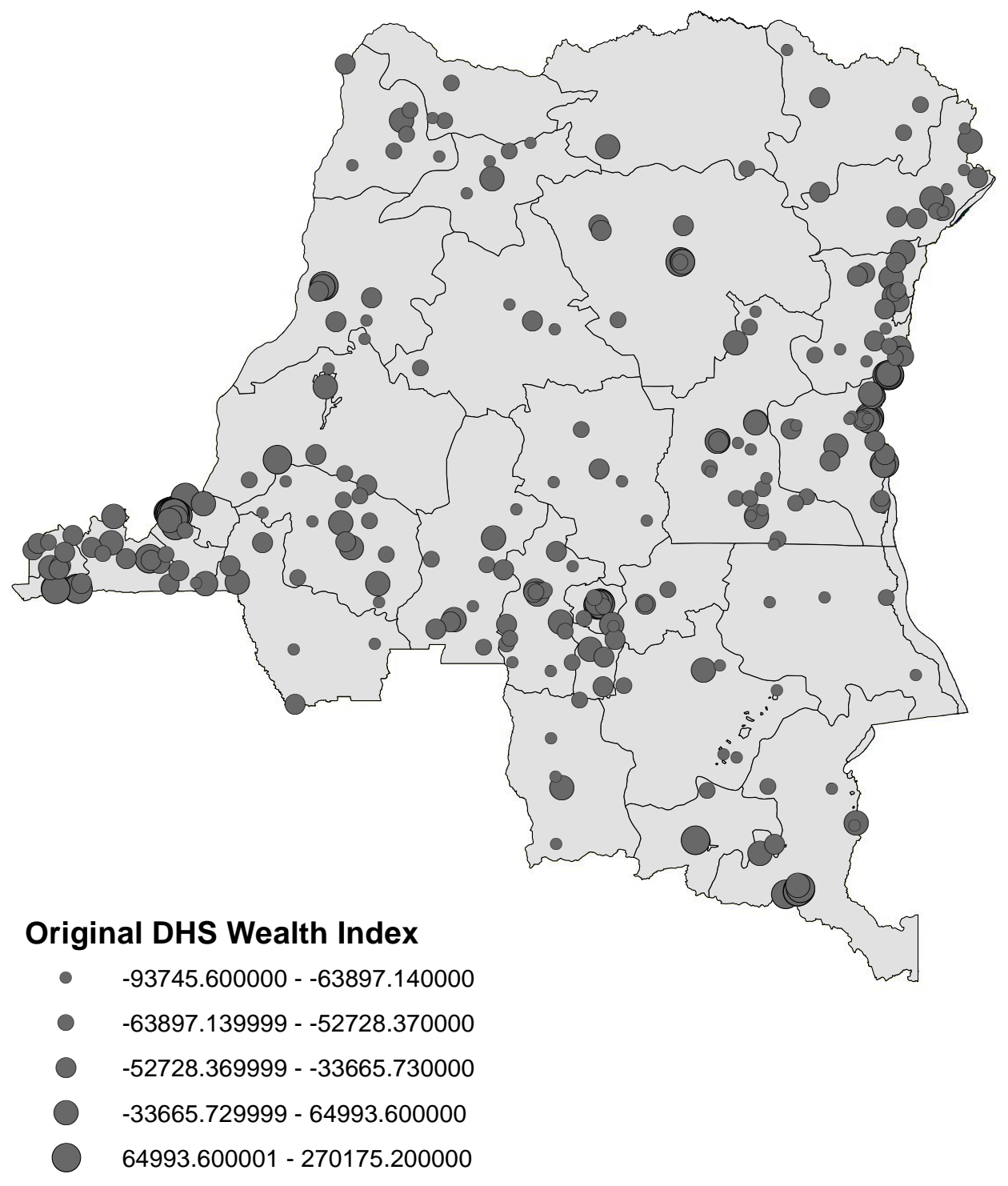




\section{Table 2.6: Instrumental Variable Regression Results - Using the Original}

DHS Wealth Index

\begin{tabular}{|c|c|c|c|c|c|c|}
\hline & $(1)$ & (2) & (3) & (4) & (5) & (6) \\
\hline Conflict & $\begin{array}{l}-88.67^{* * * *} \\
(24.34)\end{array}$ & $\begin{array}{c}-189.14^{* * * *} \\
(58.08)\end{array}$ & $\begin{array}{c}-464.17^{* * * *} \\
(175.59)\end{array}$ & $\begin{array}{l}-62.13^{* * * *} \\
(21.66)\end{array}$ & $\begin{array}{c}-137.24^{* * *} \\
(53.60)\end{array}$ & $\begin{array}{l}-324.16^{* * *} \\
(160.24)\end{array}$ \\
\hline HH Members & $\begin{array}{c}2647.15^{* * * *} \\
(433.78)\end{array}$ & $\begin{array}{c}2279.97^{* * *} \\
(438.00)\end{array}$ & $\begin{array}{c}2063.28^{* * * *} \\
(443.56)\end{array}$ & $\begin{array}{c}1981.07^{* * * *} \\
(368.45)\end{array}$ & $\begin{array}{c}1695.63^{* * * *} \\
(375.01)\end{array}$ & $\begin{array}{c}1509.81^{* * * *} \\
(377.47)\end{array}$ \\
\hline $\begin{array}{l}\text { Capital or Large } \\
\text { City }\end{array}$ & $\begin{array}{c}156401.98^{* * *} \\
(10577.93)\end{array}$ & $\begin{array}{c}133491.15^{* * * *} \\
(14847.54)\end{array}$ & $\begin{array}{l}86417.09^{* * * *} \\
(22417.62)\end{array}$ & $\begin{array}{c}136273.57^{* * * *} \\
(9915.46)\end{array}$ & $\begin{array}{c}117125.29^{* * * *} \\
(13835.52)\end{array}$ & $\begin{array}{c}77625.69^{* * * *} \\
(19896.51)\end{array}$ \\
\hline Small City & $\begin{array}{c}109743.49^{* * *} \\
(35603.25)\end{array}$ & $\begin{array}{c}120695.63^{* * * *} \\
(33047.65)\end{array}$ & $\begin{array}{c}108322.18^{* * *} \\
(32539.67)\end{array}$ & $\begin{array}{l}99338.36^{* * *} \\
(31482.17)\end{array}$ & $\begin{array}{c}110467.93^{* * *} \\
(30237.14)\end{array}$ & $\begin{array}{c}101110.88^{* * * *} \\
(30094.90)\end{array}$ \\
\hline Town & $\begin{array}{c}46398.72^{* * * *} \\
(9199.89)\end{array}$ & $\begin{array}{l}47347.82^{* * * *} \\
(10246.80)\end{array}$ & $\begin{array}{l}51860.52^{* * * *} \\
(11117.09)\end{array}$ & $\begin{array}{c}35596.71^{* * * *} \\
(8310.41)\end{array}$ & $\begin{array}{c}37800.14^{* * * *} \\
(9277.57)\end{array}$ & $\begin{array}{c}41752.67^{* * * *} \\
(9683.31)\end{array}$ \\
\hline Altitude (log) & $\begin{array}{c}1775.22 \\
(4996.68)\end{array}$ & $\begin{array}{c}2610.70 \\
(5619.77)\end{array}$ & $\begin{array}{c}5721.73 \\
(6296.50)\end{array}$ & $\begin{array}{c}1246.14 \\
(4530.12)\end{array}$ & $\begin{array}{c}1984.11 \\
(5269.91)\end{array}$ & $\begin{array}{c}4621.56 \\
(5746.98)\end{array}$ \\
\hline Under 5 Fraction & $\begin{array}{c}-38882.45^{* * *} \\
(5858.00)\end{array}$ & $\begin{array}{c}-36821.65^{* * *} \\
(5950.19)\end{array}$ & $\begin{array}{c}-36375.38^{* * * *} \\
(6315.57)\end{array}$ & $\begin{array}{c}-32547.42^{* * * *} \\
(5256.61)\end{array}$ & $\begin{array}{c}-31411.32^{* * *} \\
(5443.64)\end{array}$ & $\begin{array}{c}-30874.35^{* * * *} \\
(5685.59)\end{array}$ \\
\hline Male HHH & $\begin{array}{l}8809.40^{* * *} \\
(1967.56)\end{array}$ & $\begin{array}{c}9274.83^{* * *} \\
(1906.74)\end{array}$ & $\begin{array}{c}10104.44^{* * *} \\
(1900.10)\end{array}$ & $\begin{array}{c}-8319.77^{* * * *} \\
(2400.96)\end{array}$ & $\begin{array}{c}-6153.32^{* * * *} \\
(2364.08)\end{array}$ & $\begin{array}{l}-4899.20^{* *} \\
(2317.35)\end{array}$ \\
\hline Age of $\mathrm{HHH}$ & $\begin{array}{c}-227.85^{* * *} \\
(67.93)\end{array}$ & $\begin{array}{c}-242.38^{* * *} \\
(65.68)\end{array}$ & $\begin{array}{c}-237.04^{* * *} \\
(76.86)\end{array}$ & $\begin{array}{c}152.82^{* *} \\
(68.04)\end{array}$ & $\begin{array}{l}102.31 \\
(68.16)\end{array}$ & $\begin{array}{l}90.74 \\
(72.90)\end{array}$ \\
\hline Airports & $\begin{array}{c}27921.81^{* * * *} \\
(5875.50)\end{array}$ & $\begin{array}{c}47389.20^{* * * *} \\
(10397.38)\end{array}$ & $\begin{array}{l}99381.69^{* * * *} \\
(22202.04)\end{array}$ & $\begin{array}{c}24053.44^{* * * *} \\
(5297.99)\end{array}$ & $\begin{array}{c}41266.26^{* * *} \\
(9555.16)\end{array}$ & $\begin{array}{c}82849.79^{* * * *} \\
(20079.74)\end{array}$ \\
\hline Mining Sites & $\begin{array}{c}24831.38 \\
(19193.36)\end{array}$ & $\begin{array}{l}-61220.26^{* *} \\
(30580.44)\end{array}$ & $\begin{array}{l}-137125.73 \\
(126713.16)\end{array}$ & $\begin{array}{c}19322.68 \\
(17097.14)\end{array}$ & $\begin{array}{l}-54561.31^{* *} \\
(27370.09)\end{array}$ & $\begin{array}{l}-121728.28 \\
(113739.90)\end{array}$ \\
\hline Road Length & $\begin{array}{c}-36.83^{* * * *} \\
(8.60)\end{array}$ & $\begin{array}{c}-31.96^{* *} \\
(15.84)\end{array}$ & $\begin{array}{l}-37.17 \\
(23.50)\end{array}$ & $\begin{array}{c}-34.19^{* * *} \\
(7.87)\end{array}$ & $\begin{array}{l}-32.02^{* * *} \\
(14.58)\end{array}$ & $\begin{array}{l}-34.21 \\
(21.06)\end{array}$ \\
\hline Education of $\mathrm{HHH}$ & & & & $\begin{array}{c}4908.59^{* * *} \\
(412.56)\end{array}$ & $\begin{array}{c}4421.85^{* * *} \\
(414.67)\end{array}$ & $\begin{array}{c}4274.16^{* * *} \\
(398.45)\end{array}$ \\
\hline Distance Radius & $200 \mathrm{~km}$ & $100 \mathrm{~km}$ & $50 \mathrm{~km}$ & $200 \mathrm{~km}$ & $100 \mathrm{~km}$ & $50 \mathrm{~km}$ \\
\hline Households & 8435 & 8435 & 8435 & 8435 & 8435 & 8435 \\
\hline $\begin{array}{l}\text { F test of excluded } \\
\text { instruments }\end{array}$ & 94.07 & 87.51 & 38.68 & 93.05 & 85.39 & 37.35 \\
\hline 2nd Stage F statistic & 41.36 & 46.25 & 52.73 & 53.35 & 57.51 & 67.87 \\
\hline Distance from & $-.3045628^{* * *}$ & $-.1318439^{* * * *}$ & $-.045394^{* * *}$ & $-.30364^{* * *}$ & $-.1291978^{* * *}$ & $-.0440591^{* * *}$ \\
\hline Goma (1st Stage) & $(.0314015)$ & $(.0140939)$ & $(.0072993)$ & $(.0314783)$ & $(.0139811)$ & $(.0072088)$ \\
\hline \multicolumn{7}{|c|}{$\begin{array}{l}\text { Clustered standard errors (clustered at the DHS cluster location) in parentheses. Statistical significance is indicated as follows: } \\
{ }^{*} p<0.10,{ }^{* *} p<0.05,{ }^{* * * *} p<0.01 \text {. The dependent variable is the original DHS wealth index. Specifications (1) and (3) use a } \\
\text { measure of conflict exposure that counts all conflict events that have taken place within a } 200 \mathrm{~km} \text { radius. Specifications (2) and } \\
\text { (4) are for } 100 \mathrm{~km} \text { radii and Specifications (3) and (6) } 50 \mathrm{~km} \text { radii. A constant term is included in each specification but is not } \\
\text { reported. }\end{array}$} \\
\hline
\end{tabular}




\subsubsection{Different Instruments}

One of the potential limitations of using the distance from Goma as an instrumental variable for conflict exposure is that distance from Goma may influence household durable ownership and living conditions in other ways than its direct influence on conflict exposure. For instance, distance from Goma may capture the influence of Congolese-Rwandese trade routes or the historical provision of public goods. In this sense, the distance from Goma may very well influence household living standards in other ways. Because 'distance from Goma' is the only instrument used in the regressions above, I cannot report over identification statistics to test whether the null of instrument validity should be rejected.

In this section, I therefore explore using different instruments for conflict exposure. Instead of using distance from Goma as the sole instrument, I use the latitude coordinate and the longitude coordinate at which DHS clusters are located as instruments for cluster-region conflict exposure. These instruments are not as strongly associated with conflict exposure as the variable that captures the distances between DHS cluster locations and Goma, yet this strategy does have the advantage of allowing for the calculation of over identification statistics. Further, this approach accomplishes a similar task (albeit imperfectly) of accounting for the non-random assignment of conflict events across the country. An increase in either the latitude or longitude coordinate at which the DHS survey is located (from the average coordinates) are associated with an increase in conflict exposure. This is because, for a number of reasons discussed above, the conflict events have taken place at a much higher rate in the eastern provinces of the country. These instrumental variables regressions are modeled as follows: 


$$
\begin{aligned}
& \text { exposure }_{\mathrm{i}}=\alpha_{1}+\mathbf{x}_{\mathrm{i}}^{\prime} \beta+\mathbf{Z}_{\mathrm{i}}^{\prime} \gamma+\varepsilon_{\mathrm{i} 1} \\
& \text { wealth }_{\mathrm{i}}=\alpha_{2}+\mathbf{x}_{\mathrm{i}}^{\prime} \gamma+\eta \text { exposure } \\
& \mathrm{i}
\end{aligned}
$$

In $1.3, \mathbf{Z}_{\mathbf{i}}$ is the vector of the latitude and longitude coordinates for the DHS cluster locations and all the other variables are the same as in equations 2.1 and 2.2. One potential critique of the earlier instrumental variables approach could be that the instrument, distance from Goma, may influence the dependent variable, wealth, independent of its effects on the key explanatory variable, conflict exposure. While the validity of these instruments cannot be proven, I can now report the over identification tests (Hansen $\mathrm{J}$ statistic) from the instrumental variables regressions that use the DHS cluster location latitude and longitude coordinate to test whether the null of instrument validity should be rejected.

The estimates from the IV regressions using latitude and longitude coordinates of DHS cluster locations as separate instruments for conflict exposure are presented in Table 2.7. By and large, the coefficient estimates remain unchanged, although these aren't as strong of instruments as 'distance from Goma' (in terms of the first stage F-test of instrument exclusion). I cannot reject the null hypothesis of instrument validity in any of the specifications and the p-value for the Hansen $\mathbf{J}$ over identification statistic is quite high. In Table 2.8, I report similar results from the instrumental variables regressions that use the DHS cluster latitude and longitude coordinates as instruments, along with the original DHS wealth index. 


\section{Table 2.7: Instrumental Variable Regression Results with Latitude and Longitude Coordinates as Instruments}

\begin{tabular}{|c|c|c|c|c|c|c|}
\hline & (1) & (2) & (3) & (4) & (5) & (6) \\
\hline Conflict & $\begin{array}{c}-0.00390^{* * * *} \\
(0.00099)\end{array}$ & $\begin{array}{c}-0.00767^{* * * *} \\
(0.00190)\end{array}$ & $\begin{array}{c}-0.02102^{* * * *} \\
(0.00611)\end{array}$ & $\begin{array}{c}-0.00287^{* * * *} \\
(0.00089)\end{array}$ & $\begin{array}{c}-0.00552^{* * * *} \\
(0.00174)\end{array}$ & $\begin{array}{c}-0.01541^{* * * *} \\
(0.00541)\end{array}$ \\
\hline HH Members & $\begin{array}{c}0.15013^{* * *} \\
(0.01377)\end{array}$ & $\begin{array}{c}0.13795^{* * *} \\
(0.01382)\end{array}$ & $\begin{array}{c}0.13279^{* * *} \\
(0.01556)\end{array}$ & $\begin{array}{c}0.12812^{* * * *} \\
(0.01193)\end{array}$ & $\begin{array}{c}0.11830^{* * *} \\
(0.01202)\end{array}$ & $\begin{array}{c}0.11425^{* * *} \\
(0.01330)\end{array}$ \\
\hline Capital or Large City & $\begin{array}{c}3.49392^{* * *} \\
(0.27871)\end{array}$ & $\begin{array}{c}3.16623^{* * * *} \\
(0.38054)\end{array}$ & $\begin{array}{l}1.56811^{* * *} \\
(0.64821)\end{array}$ & $\begin{array}{c}2.84840^{* * * *} \\
(0.24685)\end{array}$ & $\begin{array}{c}2.64047^{* * * *} \\
(0.33646)\end{array}$ & $\begin{array}{l}1.32930^{* *} \\
(0.55222)\end{array}$ \\
\hline Small City & $\begin{array}{c}2.42295^{* * *} \\
(0.93531)\end{array}$ & $\begin{array}{c}2.83974^{* * *} \\
(0.75511)\end{array}$ & $\begin{array}{c}2.30503^{* * *} \\
(0.77666)\end{array}$ & $\begin{array}{c}2.12102^{* * *} \\
(0.78816)\end{array}$ & $\begin{array}{c}2.53149^{* * *} \\
(0.66514)\end{array}$ & $\begin{array}{c}2.10829^{* * *} \\
(0.68968)\end{array}$ \\
\hline Town & $\begin{array}{l}1.63771^{* * *} \\
(0.28643)\end{array}$ & $\begin{array}{l}1.68161^{* * *} \\
(0.31554)\end{array}$ & $\begin{array}{l}1.82228^{* * * *} \\
(0.37554)\end{array}$ & $\begin{array}{l}1.29286^{* * *} \\
(0.26172)\end{array}$ & $\begin{array}{l}1.37272^{* * *} \\
(0.28708)\end{array}$ & $\begin{array}{c}1.48561^{* * * *} \\
(0.32594)\end{array}$ \\
\hline Altitude (log) & $\begin{array}{c}0.35468^{*} \\
(0.19024)\end{array}$ & $\begin{array}{c}0.32843^{*} \\
(0.19189)\end{array}$ & $\begin{array}{l}0.43632^{* *} \\
(0.21265)\end{array}$ & $\begin{array}{c}0.32069^{*} \\
(0.16891)\end{array}$ & $\begin{array}{c}0.28312 \\
(0.17557)\end{array}$ & $\begin{array}{l}0.37644^{* *} \\
(0.19000)\end{array}$ \\
\hline Under 5 Fraction & $\begin{array}{c}-1.33012^{* * * *} \\
(0.19816)\end{array}$ & $\begin{array}{c}-1.31195^{* * *} \\
(0.20235)\end{array}$ & $\begin{array}{c}-1.33261^{* * * *} \\
(0.21663)\end{array}$ & $\begin{array}{c}-1.11670^{* * * *} \\
(0.18075)\end{array}$ & $\begin{array}{c}-1.12235^{* * *} \\
(0.18706)\end{array}$ & $\begin{array}{c}-1.13928^{* * * *} \\
(0.19578)\end{array}$ \\
\hline Male $\mathrm{HHH}$ & $\begin{array}{c}0.68228^{* * *} \\
(0.07576)\end{array}$ & $\begin{array}{c}0.70305^{* * *} \\
(0.07428)\end{array}$ & $\begin{array}{c}0.72368^{* * *} \\
(0.07599)\end{array}$ & $\begin{array}{c}0.12199 \\
(0.08011)\end{array}$ & $\begin{array}{l}0.18485^{* *} \\
(0.08012)\end{array}$ & $\begin{array}{c}0.21765^{* * *} \\
(0.08170)\end{array}$ \\
\hline Age of $\mathrm{HHH}$ & $\begin{array}{l}-0.00236 \\
(0.00229)\end{array}$ & $\begin{array}{l}-0.00300 \\
(0.00225)\end{array}$ & $\begin{array}{l}-0.00217 \\
(0.00265)\end{array}$ & $\begin{array}{c}0.01019^{* * * *} \\
(0.00225)\end{array}$ & $\begin{array}{c}0.00874^{* * *} \\
(0.00222)\end{array}$ & $\begin{array}{c}0.00896^{* * *} \\
(0.00245)\end{array}$ \\
\hline Airports & $\begin{array}{l}1.03673^{* * *} \\
(0.18742)\end{array}$ & $\begin{array}{l}1.34788^{* * *} \\
(0.29438)\end{array}$ & $\begin{array}{l}3.29071^{* * *} \\
(0.69227)\end{array}$ & $\begin{array}{c}0.88662^{* * * *} \\
(0.16614)\end{array}$ & $\begin{array}{l}1.09939^{* * * *} \\
(0.26517)\end{array}$ & $\begin{array}{c}2.63665^{* * *} \\
(0.61706)\end{array}$ \\
\hline Mining Sites & $\begin{array}{c}0.88626 \\
(0.74263)\end{array}$ & $\begin{array}{c}-2.36562^{* * *} \\
(0.87145)\end{array}$ & $\begin{array}{c}-5.20809 \\
(3.52354)\end{array}$ & $\begin{array}{c}0.61275 \\
(0.66979)\end{array}$ & $\begin{array}{c}-2.16064^{* * * *} \\
(0.75503)\end{array}$ & $\begin{array}{c}-4.68752 \\
(3.03376)\end{array}$ \\
\hline Road Length & $\begin{array}{c}-0.00126^{* * *} \\
(0.00030)\end{array}$ & $\begin{array}{c}0.00001 \\
(0.00047)\end{array}$ & $\begin{array}{l}-0.00003 \\
(0.00077)\end{array}$ & $\begin{array}{c}-0.00113^{* * *} \\
(0.00027)\end{array}$ & $\begin{array}{c}0.00002 \\
(0.00043)\end{array}$ & $\begin{array}{c}0.00005 \\
(0.00067)\end{array}$ \\
\hline Education of $\mathrm{HHH}$ & & & & $\begin{array}{c}0.16101^{* * *} \\
(0.01157)\end{array}$ & $\begin{array}{c}0.14868^{* * *} \\
(0.01156)\end{array}$ & $\begin{array}{c}0.14412^{* * *} \\
(0.01245)\end{array}$ \\
\hline $\begin{array}{l}\text { Distance Radius } \\
\mathrm{N}\end{array}$ & $\begin{array}{c}200 \mathrm{~km} \\
8435\end{array}$ & $\begin{array}{c}100 \mathrm{~km} \\
8435\end{array}$ & $\begin{array}{c}50 \mathrm{~km} \\
8435\end{array}$ & $\begin{array}{c}200 \mathrm{~km} \\
8435\end{array}$ & $\begin{array}{c}100 \mathrm{~km} \\
8435\end{array}$ & $\begin{array}{l}50 \mathrm{~km} \\
8435\end{array}$ \\
\hline Excluded instruments & 36.05 & 33.63 & 13.14 & 35.47 & 32.44 & 12.61 \\
\hline 2nd Stage F statistic & 58.43 & 54.98 & 55.31 & 72.10 & 69.49 & 72.06 \\
\hline Latitude ( $1^{\text {st }}$ Stage $)$ & $\begin{array}{l}19.89203^{* * * *} \\
(3.418461)\end{array}$ & $\begin{array}{l}8.384329^{* * *} \\
(1.650629)\end{array}$ & $\begin{array}{l}3.374864^{* * *} \\
(.8923943)\end{array}$ & $\begin{array}{l}19.8031^{* * *} \\
(3.415786)\end{array}$ & $\begin{array}{l}8.197676^{* * *} \\
(1.642414)\end{array}$ & $\begin{array}{l}3.282344^{* * * *} \\
(.8838805)\end{array}$ \\
\hline Longitude ( $1^{\text {st }}$ Stage $)$ & $\begin{array}{l}13.8576^{* * * *} \\
(2.986874)\end{array}$ & $\begin{array}{l}8.465902^{* * * *} \\
(1.341741)\end{array}$ & $\begin{array}{l}2.700826^{* * *} \\
(.7702219)\end{array}$ & $\begin{array}{l}13.75094^{* * * *} \\
(2.998203)\end{array}$ & $\begin{array}{l}8.276596^{* * * *} \\
(1.339882)\end{array}$ & $\begin{array}{l}2.605566^{* * * *} \\
(.7670316)\end{array}$ \\
\hline Hansen $\mathbf{J}$ - P-value & 0.7934 & 0.8725 & 0.5032 & 0.9247 & 0.8597 & 0.5108 \\
\hline
\end{tabular}


Table 2.8: Instrumental Variable Regression Results - New Instruments, DHS Wealth Index

\begin{tabular}{|c|c|c|c|c|c|c|}
\hline & (1) & (2) & (3) & (4) & (5) & (6) \\
\hline Conflict & $\begin{array}{c}-120.18^{* * *} \\
(31.75)\end{array}$ & $\begin{array}{c}-229.72^{* * * *} \\
(63.61)\end{array}$ & $\begin{array}{c}-563.81^{* * * *} \\
(187.78)\end{array}$ & $\begin{array}{c}-89.51^{k * * *} \\
(28.37)\end{array}$ & $\begin{array}{c}-167.46^{* * * *} \\
(58.09)\end{array}$ & $\begin{array}{l}-403.09^{* * *} \\
(167.81)\end{array}$ \\
\hline HH Members & $\begin{array}{c}2676.69^{* * *} \\
(454.68)\end{array}$ & $\begin{array}{c}2281.07^{* * *} \\
(453.07)\end{array}$ & $\begin{array}{c}2049.80^{* * *} \\
(469.73)\end{array}$ & $\begin{array}{c}2023.53^{* * *} \\
(385.71)\end{array}$ & $\begin{array}{c}1711.57^{* * *} \\
(386.28)\end{array}$ & $\begin{array}{c}1515.86^{* * *} \\
(396.31)\end{array}$ \\
\hline Capital or Large City & $\begin{array}{c}153571.94^{* * * *} \\
(11323.21)\end{array}$ & $\begin{array}{c}131028.33^{* * *} \\
(15702.58)\end{array}$ & $\begin{array}{c}79989.87^{* * * *} \\
(24673.46)\end{array}$ & $\begin{array}{c}134402.87^{* * *} \\
(10555.45)\end{array}$ & $\begin{array}{c}115796.99^{* * * *} \\
(14482.37)\end{array}$ & $\begin{array}{l}73054.93^{* * * *} \\
(21981.81)\end{array}$ \\
\hline Small City & $\begin{array}{c}102169.17^{* * *} \\
(37034.89)\end{array}$ & $\begin{array}{c}117115.47^{* * *} \\
(33177.99)\end{array}$ & $\begin{array}{c}103144.51^{\text {**** }} \\
(33028.28)\end{array}$ & $\begin{array}{l}93193.79^{* * * *} \\
(32727.01)\end{array}$ & $\begin{array}{c}108185.15^{* * * *} \\
(30441.44)\end{array}$ & $\begin{array}{c}97432.51^{* * * *} \\
(30554.80)\end{array}$ \\
\hline Town & $\begin{array}{c}44578.05^{* * * *} \\
(9669.73)\end{array}$ & $\begin{array}{c}46128.67^{* * * *} \\
(10647.00)\end{array}$ & $\begin{array}{l}51396.25^{* * * *} \\
(11813.34)\end{array}$ & $\begin{array}{c}34337.01^{* * * *} \\
(8739.02)\end{array}$ & $\begin{array}{c}37180.12^{* * * *} \\
(9589.86)\end{array}$ & $\begin{array}{c}41699.21^{* * * *} \\
(10261.68)\end{array}$ \\
\hline Altitude (log) & $\begin{array}{c}4975.00 \\
(5853.41)\end{array}$ & $\begin{array}{c}5045.72 \\
(6118.86)\end{array}$ & $\begin{array}{c}8262.72 \\
(6860.40)\end{array}$ & $\begin{array}{c}3970.84 \\
(5221.47)\end{array}$ & $\begin{array}{c}3733.45 \\
(5626.34)\end{array}$ & $\begin{array}{c}6562.27 \\
(6174.38)\end{array}$ \\
\hline Under 5 Fraction & $\begin{array}{c}-39963.94^{* * *} \\
(6181.09)\end{array}$ & $\begin{array}{c}-37593.52^{* * * *} \\
(6132.80)\end{array}$ & $\begin{array}{c}-37249.64^{* * * *} \\
(6444.07)\end{array}$ & $\begin{array}{c}-33629.50^{* * * *} \\
(5536.28)\end{array}$ & $\begin{array}{c}-32101.04^{* * *} \\
(5567.41)\end{array}$ & $\begin{array}{c}-31691.71^{* * * *} \\
(5745.06)\end{array}$ \\
\hline Male $\mathrm{HHH}$ & $\begin{array}{l}8509.73^{* * * *} \\
(2086.27)\end{array}$ & $\begin{array}{c}9220.22^{* * *} \\
(1979.55)\end{array}$ & $\begin{array}{c}10118.26 \\
(1960.41)\end{array}$ & $\begin{array}{c}-8125.26^{* * *} \\
(2438.69)\end{array}$ & $\begin{array}{l}-5791.95^{* *} \\
(2354.75)\end{array}$ & $\begin{array}{c}-4450.55^{* *} \\
(2226.34)\end{array}$ \\
\hline Age of $\mathrm{HHH}$ & $\begin{array}{c}-241.13^{* * * *} \\
(71.35)\end{array}$ & $\begin{array}{c}-257.72^{* * *} \\
(68.21)\end{array}$ & $\begin{array}{c}-245.76^{* * *} \\
(79.52)\end{array}$ & $\begin{array}{l}131.61^{*} \\
(69.63)\end{array}$ & $\begin{array}{c}82.45 \\
(69.09)\end{array}$ & $\begin{array}{c}74.61 \\
(72.07)\end{array}$ \\
\hline Airports & $\begin{array}{c}32391.00^{* * * *} \\
(6803.85)\end{array}$ & $\begin{array}{l}51670.93^{* * *} \\
(11291.37)\end{array}$ & $\begin{array}{c}110156.90^{* * * *} \\
(24340.56)\end{array}$ & $\begin{array}{c}27940.99^{* * *} \\
(6147.93)\end{array}$ & $\begin{array}{c}44472.54^{* * *} \\
(10341.63)\end{array}$ & $\begin{array}{l}91426.10^{* * *} \\
(22020.86)\end{array}$ \\
\hline Mining Sites & $\begin{array}{c}42689.42^{*} \\
(22873.22)\end{array}$ & $\begin{array}{l}-59319.97^{*} \\
(32093.81)\end{array}$ & $\begin{array}{c}-137280.54 \\
(132946.06)\end{array}$ & $\begin{array}{c}34596.18^{*} \\
(20441.96)\end{array}$ & $\begin{array}{l}-53381.56^{*} \\
(28542.66)\end{array}$ & $\begin{array}{c}-122294.34 \\
(118753.13)\end{array}$ \\
\hline Road Length & $\begin{array}{c}-44.21^{* * *} \\
(9.99)\end{array}$ & $\begin{array}{l}-32.84^{* *} \\
(16.44)\end{array}$ & $\begin{array}{l}-35.08 \\
(24.94)\end{array}$ & $\begin{array}{c}-40.51^{* * *} \\
(9.04)\end{array}$ & $\begin{array}{l}-32.65^{* * *} \\
(15.02)\end{array}$ & $\begin{array}{l}-32.73 \\
(22.20)\end{array}$ \\
\hline Education of $\mathrm{HHH}$ & & & & $\begin{array}{c}4780.10^{* * * *} \\
(407.98)\end{array}$ & $\begin{array}{c}4307.13^{* * *} \\
(410.32)\end{array}$ & $\begin{array}{c}4149.31^{* * *} \\
(386.08)\end{array}$ \\
\hline Distance Radius & $200 \mathrm{~km}$ & $100 \mathrm{~km}$ & $50 \mathrm{~km}$ & $200 \mathrm{~km}$ & $100 \mathrm{~km}$ & $50 \mathrm{~km}$ \\
\hline Households & 8435 & 8435 & 8435 & 8435 & 8435 & 8435 \\
\hline Excluded instruments & 36.05 & 33.63 & 13.14 & 35.47 & 32.44 & 12.61 \\
\hline 2nd Stage F statistic & 39.39 & 44.40 & 51.24 & 49.47 & 54.87 & 64.53 \\
\hline Latitude (1st Stage) & $\begin{array}{l}19.89203^{* * *} \\
(3.418461)\end{array}$ & $\begin{array}{l}8.384329^{* * * *} \\
(1.650629)\end{array}$ & $\begin{array}{l}3.374864^{* * *} \\
(.8923943)\end{array}$ & $\begin{array}{l}19.8031^{* * * *} \\
(3.415786)\end{array}$ & $\begin{array}{l}8.197676^{* * *} \\
(1.642414)\end{array}$ & $\begin{array}{c}3.282344^{* * * *} \\
(.8838805)\end{array}$ \\
\hline Longitude (1st Stage) & $\begin{array}{l}13.8576^{* * *} \\
(2.986874)\end{array}$ & $\begin{array}{l}8.465902^{* * *} \\
(1.341741)\end{array}$ & $\begin{array}{c}2.700826^{* * *} \\
(.7702219)\end{array}$ & $\begin{array}{l}13.75094^{* * *} \\
(2.998203)\end{array}$ & $\begin{array}{c}8.276596^{* * * *} \\
(1.339882)\end{array}$ & $\begin{array}{c}2.605566^{* * *} \\
(.7670316)\end{array}$ \\
\hline Hansen $\mathbf{J}$ - P-value & 0.3960 & 0.1798 & 0.3440 & 0.2551 & 0.1431 & 0.2571 \\
\hline
\end{tabular}

Clustered standard errors (clustered at the DHS cluster location) in parentheses. Statistical significance is indicated as follows: ${ }^{*} p<0.10,{ }^{* *} p<0.05,{ }^{* * *} p<0.01$. The dependent variable is the original DHS wealth index. Specifications (1) and (3) use a measure of conflict exposure that counts all conflict events that have taken place within a $200 \mathrm{~km}$ radius. Specifications (2) and (4) are for $100 \mathrm{~km}$ radii and Specifications (3) and (6) $50 \mathrm{~km}$ radii. A constant term is included in each specification but is not reported. 


\subsubsection{Splitting the Wealth Index into Two Components}

It is also interesting to tease out what specific economic impacts the civil wars and conflict events have had. In Table 2.9 and Table 2.10, I split the wealth index into two different measures: one wealth index that measures relatively more "liquid" household durables or assets and another that measures more "illiquid" household durables or assets. Specifically, I define the liquid asset index as a simple additive measure of the following indicators:

- Television

- Radio

- Bicycle

- Mobile phone

- Motor transport (car, truck, motorcycle, or scooter)

- Chairs

- Lamps

- Hoes

I define the 'illiquid' durables or measures of living conditions as an addition of the following indicators:

- Electricity

- Cement floor

- Improved water source

- Refrigerator

- Telephone

- Improved toilet

- Beds

- Stove/cooker

- Grill/heater

By and large, I find similar results when splitting the wealth index into its 'liquid' and 'illiquid' components (see Table 2.9 and Table 2.10). This suggests that both household-level durable ownership and household-level living conditions have been affected by conflict and local insecurity. 
Table 2.9: Instrumental Variable Regression Results - Wealth Index using "Liquid" Components Only

\begin{tabular}{|c|c|c|c|c|c|c|}
\hline & $(1)$ & $(2)$ & (3) & (4) & $(5)$ & $(6)$ \\
\hline Conflict & $\begin{array}{c}-0.00206^{* * * *} \\
(0.00057)\end{array}$ & $\begin{array}{c}-0.00399^{* * * *} \\
(0.00103)\end{array}$ & $\begin{array}{c}-0.01201^{* * * *} \\
(0.00351)\end{array}$ & $\begin{array}{c}-0.00147^{* * * *} \\
(0.00053)\end{array}$ & $\begin{array}{c}-0.00272^{* * * *} \\
(0.00097)\end{array}$ & $\begin{array}{c}-0.00867^{* * * *} \\
(0.00314)\end{array}$ \\
\hline HH Members & $\begin{array}{c}0.10568^{* * *} \\
(0.00803)\end{array}$ & $\begin{array}{c}0.10075^{* * * *} \\
(0.00795)\end{array}$ & $\begin{array}{c}0.10002^{* * * *} \\
(0.00916)\end{array}$ & $\begin{array}{c}0.09314^{* * * *} \\
(0.00728)\end{array}$ & $\begin{array}{c}0.08912^{* * *} \\
(0.00719)\end{array}$ & $\begin{array}{c}0.08900^{* * *} \\
(0.00802)\end{array}$ \\
\hline Capital or Large City & $\begin{array}{l}1.22976^{* * *} \\
(0.12548)\end{array}$ & $\begin{array}{l}1.29553^{* * * *} \\
(0.15366)\end{array}$ & $\begin{array}{l}0.60655^{* *} \\
(0.27865)\end{array}$ & $\begin{array}{c}0.86196^{* * * *} \\
(0.11197)\end{array}$ & $\begin{array}{c}0.98446^{* * *} \\
(0.13449)\end{array}$ & $\begin{array}{l}0.46492^{* *} \\
(0.22693)\end{array}$ \\
\hline Small City & $\begin{array}{l}0.96937^{* *} \\
(0.38108)\end{array}$ & $\begin{array}{l}1.15399^{* * * *} \\
(0.25498)\end{array}$ & $\begin{array}{c}0.86689^{* * * *} \\
(0.30866)\end{array}$ & $\begin{array}{c}0.79737^{\text {**** }} \\
(0.30599)\end{array}$ & $\begin{array}{c}0.97162^{* * * *} \\
(0.21183)\end{array}$ & $\begin{array}{c}0.75021^{* * * *} \\
(0.26367)\end{array}$ \\
\hline Town & $\begin{array}{c}0.91681^{* * *} \\
(0.16728)\end{array}$ & $\begin{array}{c}0.93525^{* * *} \\
(0.17150)\end{array}$ & $\begin{array}{c}0.99118^{* * *} \\
(0.20945)\end{array}$ & $\begin{array}{c}0.72032^{* * * *} \\
(0.15289)\end{array}$ & $\begin{array}{c}0.75250^{* * *} \\
(0.15552)\end{array}$ & $\begin{array}{c}0.79116^{* * *} \\
(0.17995)\end{array}$ \\
\hline Altitude (log) & $\begin{array}{l}0.26675^{* *} \\
(0.11498)\end{array}$ & $\begin{array}{l}0.24253^{* *} \\
(0.10754)\end{array}$ & $\begin{array}{l}0.29934^{* *} \\
(0.11787)\end{array}$ & $\begin{array}{l}0.24736^{* *} \\
(0.10457)\end{array}$ & $\begin{array}{l}0.21572^{* *} \\
(0.09982)\end{array}$ & $\begin{array}{l}0.26365^{* *} \\
(0.10687)\end{array}$ \\
\hline Under 5 Fraction & $\begin{array}{c}-0.68881^{* * *} \\
(0.12013)\end{array}$ & $\begin{array}{c}-0.705666^{* * *} \\
(0.12228)\end{array}$ & $\begin{array}{c}-0.73426^{* * *} \\
(0.12945)\end{array}$ & $\begin{array}{c}-0.56720^{* * *} \\
(0.11409)\end{array}$ & $\begin{array}{c}-0.59348^{* * *} \\
(0.11672)\end{array}$ & $\begin{array}{c}-0.61935^{* * *} \\
(0.12053)\end{array}$ \\
\hline Male HHH & $\begin{array}{c}0.58246^{* * *} \\
(0.04943)\end{array}$ & $\begin{array}{c}0.59294^{* * * *} \\
(0.04869)\end{array}$ & $\begin{array}{c}0.59826^{* * *} \\
(0.05077)\end{array}$ & $\begin{array}{c}0.26320^{\text {**** }} \\
(0.05171)\end{array}$ & $\begin{array}{c}0.28634^{* * *} \\
(0.05258)\end{array}$ & $\begin{array}{c}0.29757^{* * *} \\
(0.05479)\end{array}$ \\
\hline Age of $\mathrm{HHH}$ & $\begin{array}{l}-0.00048 \\
(0.00137)\end{array}$ & $\begin{array}{l}-0.00086 \\
(0.00134)\end{array}$ & $\begin{array}{l}-0.00024 \\
(0.00150)\end{array}$ & $\begin{array}{c}0.00667^{* * * *} \\
(0.00135)\end{array}$ & $\begin{array}{c}0.00608^{* * * *} \\
(0.00133)\end{array}$ & $\begin{array}{c}0.00638^{* * * *} \\
(0.00144)\end{array}$ \\
\hline Airports & $\begin{array}{c}0.44579^{* * * *} \\
(0.10002)\end{array}$ & $\begin{array}{c}0.44736^{* * * *} \\
(0.13358)\end{array}$ & $\begin{array}{l}1.39671^{* * *} \\
(0.34561)\end{array}$ & $\begin{array}{c}0.36023^{* * * *} \\
(0.09133)\end{array}$ & $\begin{array}{l}0.30034^{* *} \\
(0.12342)\end{array}$ & $\begin{array}{l}1.00762^{* * * *} \\
(0.31063)\end{array}$ \\
\hline Mining Sites & $\begin{array}{c}0.36095 \\
(0.44053)\end{array}$ & $\begin{array}{c}-1.24577^{* * *} \\
(0.40071)\end{array}$ & $\begin{array}{c}-3.17861^{* *} \\
(1.56705)\end{array}$ & $\begin{array}{c}0.20499 \\
(0.40693)\end{array}$ & $\begin{array}{c}-1.12450^{* * *} \\
(0.34725)\end{array}$ & $\begin{array}{c}-2.86928^{* *} \\
(1.31977)\end{array}$ \\
\hline Road Length & $\begin{array}{c}-0.00051^{* * * *} \\
(0.00017)\end{array}$ & $\begin{array}{l}0.00053^{* *} \\
(0.00022)\end{array}$ & $\begin{array}{c}0.00043 \\
(0.00040)\end{array}$ & $\begin{array}{c}-0.00044^{* * * *} \\
(0.00016)\end{array}$ & $\begin{array}{c}0.00054^{* * *} \\
(0.00020)\end{array}$ & $\begin{array}{c}0.00048 \\
(0.00035)\end{array}$ \\
\hline Education of $\mathrm{HHH}$ & & & & $\begin{array}{c}0.09174^{* * *} \\
(0.00668)\end{array}$ & $\begin{array}{c}0.08797^{* * *} \\
(0.00663)\end{array}$ & $\begin{array}{c}0.08564^{* * *} \\
(0.00755)\end{array}$ \\
\hline Distance Radius & $200 \mathrm{~km}$ & $100 \mathrm{~km}$ & $50 \mathrm{~km}$ & $200 \mathrm{~km}$ & $100 \mathrm{~km}$ & $50 \mathrm{~km}$ \\
\hline Households & 8435 & 8435 & 8435 & 8435 & 8435 & 8435 \\
\hline Excluded instruments & 36.05 & 33.63 & 13.14 & 35.47 & 32.44 & 12.61 \\
\hline 2nd Stage F statistic & 73.05 & 69.74 & 61.75 & 91.95 & 87.93 & 78.86 \\
\hline Latitude (1st Stage) & $\begin{array}{c}19.89203^{* * *} \\
(3.418461)\end{array}$ & $\begin{array}{c}8.384329^{\text {**** }} \\
(1.650629)\end{array}$ & $\begin{array}{c}3.374864^{* * * *} \\
(.8923943)\end{array}$ & $\begin{array}{l}19.8031^{* * *} \\
(3.415786)\end{array}$ & $\begin{array}{c}8.197676^{* * *} \\
(1.642414)\end{array}$ & $\begin{array}{c}3.282344^{* * *} \\
(.8838805)\end{array}$ \\
\hline Longitude (1st Stage) & $\begin{array}{l}13.8576^{* * *} \\
(2.986874)\end{array}$ & $\begin{array}{l}8.465902^{* * * *} \\
(1.341741)\end{array}$ & $\begin{array}{l}2.700826^{* * * *} \\
(.7702219)\end{array}$ & $\begin{array}{l}13.75094^{* * * *} \\
(2.998203)\end{array}$ & $\begin{array}{l}8.276596^{* * * *} \\
(1.339882)\end{array}$ & $\begin{array}{l}2.605566^{* * * *} \\
(.7670316)\end{array}$ \\
\hline Hansen J - P-value & 0.5657 & 0.4726 & 0.2458 & 0.6669 & 0.4232 & 0.2271 \\
\hline
\end{tabular}

Clustered standard errors (clustered at the DHS cluster location) in parentheses. Statistical significance is indicated as follows: ${ }^{*} p<0.10,{ }^{* *} p<0.05,{ }^{* * *} p<0.01$. The dependent variable is the original DHS wealth index. Specifications (1) and (3) use a measure of conflict exposure that counts all conflict events that have taken place within a $200 \mathrm{~km}$ radius. Specifications (2) and (4) are for $100 \mathrm{~km}$ radii and Specifications (3) and (6) $50 \mathrm{~km}$ radii. A constant term is included in each specification but is not reported. 


\begin{tabular}{|c|c|c|c|c|c|c|}
\hline & (1) & (2) & (3) & (4) & (5) & (6) \\
\hline Conflict & $\begin{array}{c}-0.00184^{* * * *} \\
(0.00055)\end{array}$ & $\begin{array}{c}-0.00368^{* * * *} \\
(0.00111)\end{array}$ & $\begin{array}{c}-0.00901^{* * * *} \\
(0.00317)\end{array}$ & $\begin{array}{c}-0.00140^{* * * *} \\
(0.00051)\end{array}$ & $\begin{array}{c}-0.00281^{* * * *} \\
(0.00105)\end{array}$ & $\begin{array}{l}-0.00673^{* * *} \\
(0.00296)\end{array}$ \\
\hline HH Members & $\begin{array}{c}0.04444^{* * *} \\
(0.00794)\end{array}$ & $\begin{array}{c}0.03720^{* * * *} \\
(0.00808)\end{array}$ & $\begin{array}{c}0.03277^{* * *} \\
(0.00820)\end{array}$ & $\begin{array}{c}0.03498^{* * * *} \\
(0.00696)\end{array}$ & $\begin{array}{c}0.02917^{* * *} \\
(0.00713)\end{array}$ & $\begin{array}{l}0.02525^{* * *} \\
(0.00719)\end{array}$ \\
\hline Capital or Large City & $\begin{array}{l}2.26416^{* * *} \\
(0.19562)\end{array}$ & $\begin{array}{l}1.87070^{* * *} \\
(0.27062)\end{array}$ & $\begin{array}{l}0.96156^{* *} \\
(0.41610)\end{array}$ & $\begin{array}{l}1.98644^{* * *} \\
(0.18359)\end{array}$ & $\begin{array}{l}1.65601^{* * *} \\
(0.25250)\end{array}$ & $\begin{array}{l}0.86438^{* *} \\
(0.37908)\end{array}$ \\
\hline Small City & $\begin{array}{l}1.45358^{* *} \\
(0.61286)\end{array}$ & $\begin{array}{c}1.68575^{* * *} \\
(0.53751)\end{array}$ & $\begin{array}{l}1.43813^{* * *} \\
(0.53513)\end{array}$ & $\begin{array}{l}1.32364^{* * *} \\
(0.55232)\end{array}$ & $\begin{array}{l}1.55988^{* * *} \\
(0.50017)\end{array}$ & $\begin{array}{c}1.35808^{* * *} \\
(0.50153)\end{array}$ \\
\hline Town & $\begin{array}{c}0.72090^{* * *} \\
(0.15058)\end{array}$ & $\begin{array}{c}0.74636^{* * *} \\
(0.17511)\end{array}$ & $\begin{array}{c}0.83110^{* * *} \\
(0.19090)\end{array}$ & $\begin{array}{c}0.57254^{* * *} \\
(0.14237)\end{array}$ & $\begin{array}{c}0.62023^{* * *} \\
(0.16490)\end{array}$ & $\begin{array}{c}0.69446^{* * *} \\
(0.17355)\end{array}$ \\
\hline Altitude (log) & $\begin{array}{c}0.08793 \\
(0.10166)\end{array}$ & $\begin{array}{c}0.08590 \\
(0.10874)\end{array}$ & $\begin{array}{c}0.13697 \\
(0.12046)\end{array}$ & $\begin{array}{c}0.07333 \\
(0.09358)\end{array}$ & $\begin{array}{c}0.06740 \\
(0.10251)\end{array}$ & $\begin{array}{c}0.11278 \\
(0.11198)\end{array}$ \\
\hline Under 5 Fraction & $\begin{array}{c}-0.64131^{* * *} \\
(0.11451)\end{array}$ & $\begin{array}{c}-0.60629^{* * * *} \\
(0.11400)\end{array}$ & $\begin{array}{c}-0.59835^{* * *} \\
(0.11875)\end{array}$ & $\begin{array}{c}-0.54950^{* * * *} \\
(0.10456)\end{array}$ & $\begin{array}{c}-0.52887^{* * * *} \\
(0.10547)\end{array}$ & $\begin{array}{c}-0.51993^{* * *} \\
(0.10873)\end{array}$ \\
\hline Male HHH & $\begin{array}{l}0.09982^{* *} \\
(0.04000)\end{array}$ & $\begin{array}{c}0.11012^{* * * *} \\
(0.03922)\end{array}$ & $\begin{array}{c}0.12543^{* * *} \\
(0.03780)\end{array}$ & $\begin{array}{c}-0.14121^{* * *} \\
(0.04814)\end{array}$ & $\begin{array}{l}-0.10149^{* *} \\
(0.04714)\end{array}$ & $\begin{array}{l}-0.07993^{*} \\
(0.04471)\end{array}$ \\
\hline Age of $\mathrm{HHH}$ & $\begin{array}{l}-0.00188 \\
(0.00132)\end{array}$ & $\begin{array}{l}-0.00214^{*} \\
(0.00128)\end{array}$ & $\begin{array}{l}-0.00193 \\
(0.00146)\end{array}$ & $\begin{array}{c}0.00352^{* * * *} \\
(0.00134)\end{array}$ & $\begin{array}{l}0.00266^{* *} \\
(0.00131)\end{array}$ & $\begin{array}{c}0.00258^{*} \\
(0.00137)\end{array}$ \\
\hline Airports & $\begin{array}{l}0.59094^{* * *} \\
(0.11911)\end{array}$ & $\begin{array}{c}0.90052^{* * *} \\
(0.19972)\end{array}$ & $\begin{array}{l}1.89400^{* * *} \\
(0.41749)\end{array}$ & $\begin{array}{c}0.52639^{* * * *} \\
(0.11043)\end{array}$ & $\begin{array}{c}0.79905^{* * *} \\
(0.18852)\end{array}$ & $\begin{array}{l}1.62904^{* * *} \\
(0.39145)\end{array}$ \\
\hline Mining Sites & $\begin{array}{c}0.52531 \\
(0.40254)\end{array}$ & $\begin{array}{l}-1.11985^{* *} \\
(0.56520)\end{array}$ & $\begin{array}{l}-2.02948 \\
(2.30184)\end{array}$ & $\begin{array}{c}0.40776 \\
(0.37266)\end{array}$ & $\begin{array}{c}-1.03614^{* *} \\
(0.51965)\end{array}$ & $\begin{array}{c}-1.81824 \\
(2.11763)\end{array}$ \\
\hline Road Length & $\begin{array}{c}-0.00074^{* * *} \\
(0.00017)\end{array}$ & $\begin{array}{l}-0.00052^{*} \\
(0.00030)\end{array}$ & $\begin{array}{l}-0.00046 \\
(0.00043)\end{array}$ & $\begin{array}{c}-0.00069^{* * *} \\
(0.00016)\end{array}$ & $\begin{array}{l}-0.00052^{*} \\
(0.00028)\end{array}$ & $\begin{array}{l}-0.00043 \\
(0.00040)\end{array}$ \\
\hline Education of $\mathrm{HHH}$ & & & & $\begin{array}{c}0.06926^{* * *} \\
(0.00764)\end{array}$ & $\begin{array}{c}0.06071^{* * *} \\
(0.00773) \\
\end{array}$ & $\begin{array}{c}0.05849^{* * *} \\
(0.00734)\end{array}$ \\
\hline Distance Radius & $200 \mathrm{~km}$ & $100 \mathrm{~km}$ & $50 \mathrm{~km}$ & $200 \mathrm{~km}$ & $100 \mathrm{~km}$ & $50 \mathrm{~km}$ \\
\hline Households & 8435 & 8435 & 8435 & 8435 & 8435 & 8435 \\
\hline Excluded instruments & 36.05 & 33.63 & 13.14 & 35.47 & 32.44 & 12.61 \\
\hline 2nd Stage F statistic & 28.52 & 30.23 & 33.82 & 31.69 & 33.57 & 38.16 \\
\hline Latitude (1st Stage) & $\begin{array}{l}19.89203^{* * * *} \\
(3.418461)\end{array}$ & $\begin{array}{l}8.384329^{* * *} \\
(1.650629)\end{array}$ & $\begin{array}{c}3.374864^{* * *} \\
(.8923943)\end{array}$ & $\begin{array}{l}19.8031^{* * *} \\
(3.415786)\end{array}$ & $\begin{array}{l}8.197676^{* * * *} \\
(1.642414)\end{array}$ & $\begin{array}{l}3.282344^{* * * *} \\
(.8838805)\end{array}$ \\
\hline Longitude (1st Stage) & $\begin{array}{l}13.8576^{* * * *} \\
(2.986874)\end{array}$ & $\begin{array}{l}8.465902^{* * *} \\
(1.341741)\end{array}$ & $\begin{array}{l}2.700826^{* * *} \\
(.7702219)\end{array}$ & $\begin{array}{l}13.75094^{* * *} \\
(2.998203)\end{array}$ & $\begin{array}{l}8.276596^{* * *} \\
(1.339882)\end{array}$ & $\begin{array}{c}2.605566^{* * *} \\
(.7670316)\end{array}$ \\
\hline Hansen J - P-value & 0.9491 & 0.7227 & 0.9375 & 0.8307 & 0.7044 & 0.9869 \\
\hline \multicolumn{7}{|c|}{$\begin{array}{l}\text { Clustered standard errors (clustered at the DHS cluster location) in parentheses. Statistical significance is indicated as follows: } \\
{ }^{*} p<0.10,{ }^{* *} p<0.05,{ }^{* * *} p<0.01 \text {. The dependent variable is the original DHS wealth index. Specifications (1) and (3) use a } \\
\text { measure of conflict exposure that counts all conflict events that have taken place within a } 200 \mathrm{~km} \text { radius. Specifications (2) and } \\
\text { (4) are for } 100 \mathrm{~km} \text { radii and Specifications (3) and (6) } 50 \mathrm{~km} \text { radii. A constant term is included in each specification but is not } \\
\text { reported. }\end{array}$} \\
\hline
\end{tabular}




\subsubsection{New Data on Conflict Events}

The Uppsala Conflict Data Program (UCDP) recently released the Georeferenced Events Dataset (GED) (Sundberg, Lindgren, and Padskocimaite, 2010; Melander and Sundberg, 2011). Much like the ACLED, the GED provides georeferenced, disaggregated information on conflict events. The scatterplots of conflict exposure and distance from Goma, using the UCDP-GED data, are presented in Figure 2.5 and the geographic distribution and intensity of conflict events, as measured by the UCDP-GED, are presented in Figure 2.6. Unlike the ACLED, however, the GED covers a time period from 1989 onwards and has different guidelines for how conflict events are defined and recorded. Because the ACLED may have been developed with some degree of measurement error (Eck, 2012), it is important to assess whether this influences the core results in this paper. While it is beyond the scope of this paper to compare and contrast the GED and ACLED, interested readers can find an article-length comparison of the two datasets in Eck (2012).

For the purposes of this paper, I will use the GED to demonstrate that regardless of how conflict events across the DRC are defined and coded, I find similar results regarding the impact of conflict and local insecurity on household-level economic conditions across the country. Again, I use the ACLED and GED conflict events datasets mainly as proxies for local and regional conflict exposure, so the individual events and how they are coded and defined are of less concern than the local and regional aggregated conflict exposure measures.

I created $200 \mathrm{~km}, 100 \mathrm{~km}$, and $50 \mathrm{~km}$ buffers around DHS cluster locations and then considered how many conflict events were contained within each buffer zone. Remarkably, in doing so I find correlations of the ACLED and GED conflict exposure measures of 0.9761 for a $200 \mathrm{~km}$ radius, 0.9405 for a $100 \mathrm{~km}$ radius, and 0.9245 for a $50 \mathrm{~km}$ radius. These correlations 
suggest that both measures of conflict exposure are essentially capturing the same effects and both therefore measures serve as reasonable proxies for local conflict exposure and local insecurity. This is surprising considering that the two datasets have different temporal dimensions - the GED has a longer time frame than the ACLED - and there are more events coded in the ACLED than in the GED. Results are presented in Table 2.11, showing very similar relative magnitudes of the impact of conflict and local insecurity on household-level durable ownership and living conditions.

\section{Figure 2.5: Scatterplots of Conflict Exposure (UCDP-GED) and Distance from Goma}

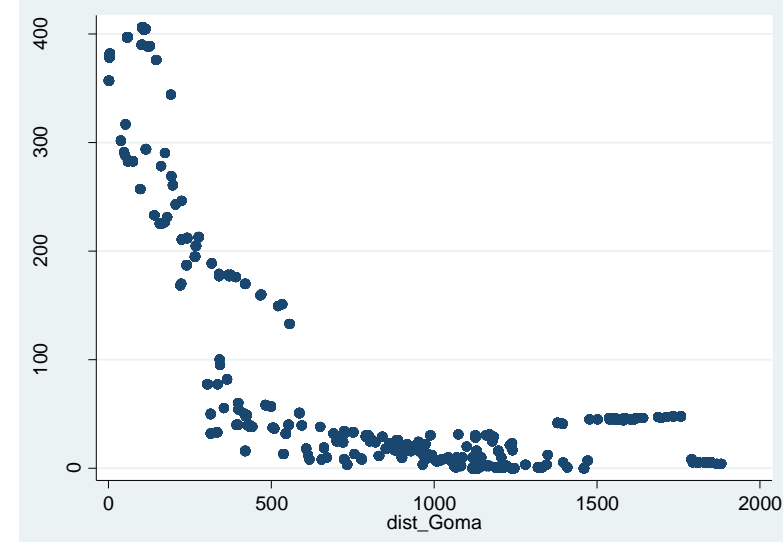

Conflict exposure within $200 \mathrm{~km}$

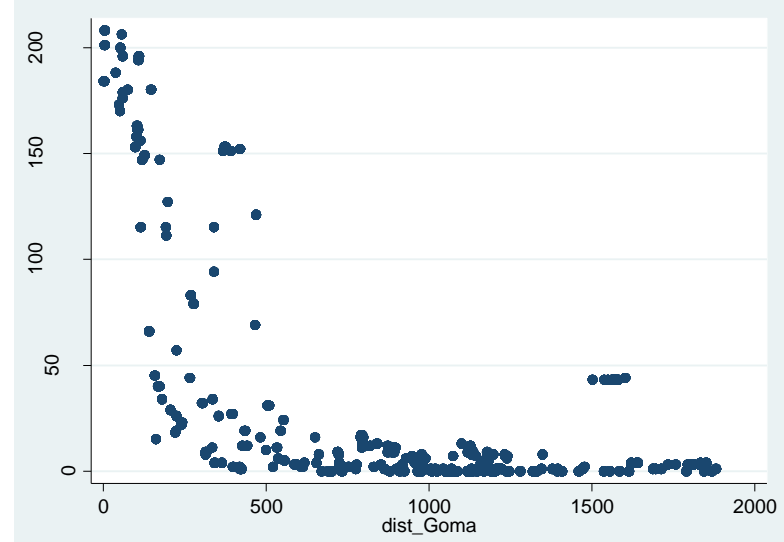

Conflict Exposure within 100 km

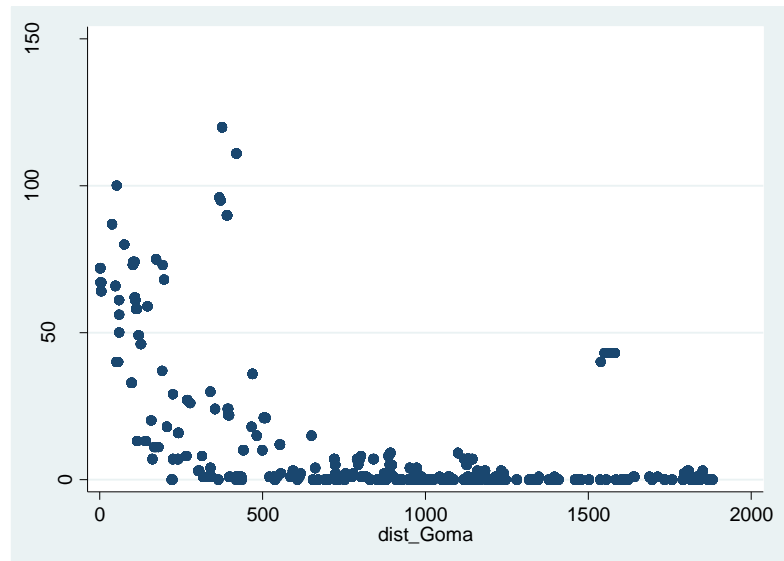

Conflict Exposure within 50 km 
Figure 2.6: Distribution (By Quintile) of Conflict Events (UCDP-GED Data)

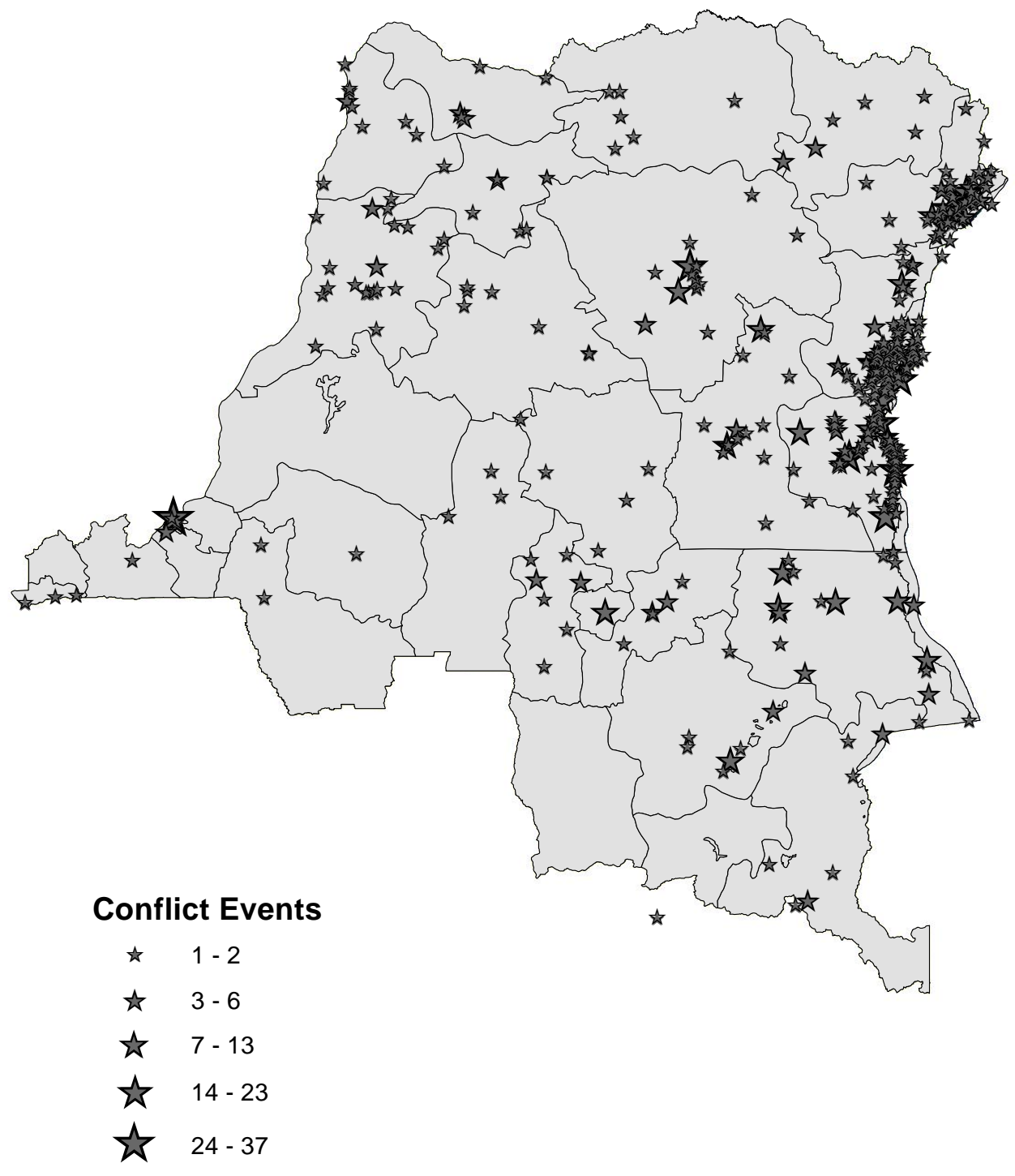


Table 2.11: Instrumental Variable Regression Results - UCDP-GED Data

\begin{tabular}{|c|c|c|c|c|c|c|}
\hline & (1) & (2) & (3) & (4) & (5) & (6) \\
\hline Conflict & $\begin{array}{c}-0.01008^{* * * *} \\
(0.00238)\end{array}$ & $\begin{array}{c}-0.01779^{* * *} \\
(0.00405)\end{array}$ & $\begin{array}{c}-0.05072^{* * * *} \\
(0.01299)\end{array}$ & $\begin{array}{c}-0.00743^{* * * *} \\
(0.00219)\end{array}$ & $\begin{array}{c}-0.01283^{* * *} \\
(0.00377)\end{array}$ & $\begin{array}{c}-0.03709^{* * * *} \\
(0.01171)\end{array}$ \\
\hline HH Members & $\begin{array}{c}0.15443^{* * *} \\
(0.01354)\end{array}$ & $\begin{array}{c}0.13741^{* * *} \\
(0.01338)\end{array}$ & $\begin{array}{c}0.13442^{* * *} \\
(0.01395)\end{array}$ & $\begin{array}{c}0.13153^{* * *} \\
(0.01179)\end{array}$ & $\begin{array}{c}0.11795^{* * *} \\
(0.01175)\end{array}$ & $\begin{array}{c}0.11522^{* * *} \\
(0.01222)\end{array}$ \\
\hline Capital or Large City & $\begin{array}{l}3.50074^{* * * *} \\
(0.27792)\end{array}$ & $\begin{array}{c}3.12009^{* * * *} \\
(0.36171)\end{array}$ & $\begin{array}{c}2.10048^{* * * *} \\
(0.51335)\end{array}$ & $\begin{array}{c}2.86034^{* * * *} \\
(0.24664)\end{array}$ & $\begin{array}{c}2.60797^{\text {**** }} \\
(0.32316)\end{array}$ & $\begin{array}{l}1.71379^{* * * *} \\
(0.44187)\end{array}$ \\
\hline Small City & $\begin{array}{c}2.48055^{* * *} \\
(0.92522)\end{array}$ & $\begin{array}{c}2.87878^{* * * *} \\
(0.74914)\end{array}$ & $\begin{array}{c}2.51070^{* * * *} \\
(0.75404)\end{array}$ & $\begin{array}{c}2.16692^{* * *} \\
(0.78070)\end{array}$ & $\begin{array}{c}2.55976^{* * *} \\
(0.65901)\end{array}$ & $\begin{array}{c}2.25476^{* * * *} \\
(0.66845)\end{array}$ \\
\hline Town & $\begin{array}{l}1.60787^{* * *} \\
(0.27498)\end{array}$ & $\begin{array}{l}1.75295^{* * *} \\
(0.30481)\end{array}$ & $\begin{array}{l}1.86188^{* * *} \\
(0.33740)\end{array}$ & $\begin{array}{l}1.27455^{* * *} \\
(0.25249)\end{array}$ & $\begin{array}{l}1.42468^{* * *} \\
(0.27823)\end{array}$ & $\begin{array}{l}1.51050^{* * *} \\
(0.29803)\end{array}$ \\
\hline Altitude (log) & $\begin{array}{l}0.31534^{*} \\
(0.17888)\end{array}$ & $\begin{array}{l}0.34298^{*} \\
(0.19136)\end{array}$ & $\begin{array}{l}0.44701^{* * *} \\
(0.21193)\end{array}$ & $\begin{array}{l}0.29195^{*} \\
(0.16026)\end{array}$ & $\begin{array}{l}0.29411^{*} \\
(0.17466)\end{array}$ & $\begin{array}{l}0.384366^{* *} \\
(0.18841)\end{array}$ \\
\hline Under 5 Fraction & $\begin{array}{c}-1.33359^{* * *} \\
(0.19369)\end{array}$ & $\begin{array}{c}-1.28400^{* * *} \\
(0.19060)\end{array}$ & $\begin{array}{c}-1.35066^{* * *} \\
(0.20598)\end{array}$ & $\begin{array}{c}-1.12148^{* * *} \\
(0.17734)\end{array}$ & $\begin{array}{c}-1.10275^{* * *} \\
(0.17825)\end{array}$ & $\begin{array}{c}-1.15050^{* * *} \\
(0.18856)\end{array}$ \\
\hline Male $\mathrm{HHH}$ & $\begin{array}{c}0.67451^{* * *} \\
(0.07515)\end{array}$ & $\begin{array}{c}0.70718^{* * * *} \\
(0.07079)\end{array}$ & $\begin{array}{c}0.72255^{* * * *} \\
(0.07070)\end{array}$ & $\begin{array}{c}0.12219 \\
(0.07916)\end{array}$ & $\begin{array}{l}0.18898^{* *} \\
(0.07861)\end{array}$ & $\begin{array}{c}0.21092^{* * *} \\
(0.07897)\end{array}$ \\
\hline Age of $\mathrm{HHH}$ & $\begin{array}{l}-0.00304 \\
(0.00227)\end{array}$ & $\begin{array}{l}-0.00319 \\
(0.00218)\end{array}$ & $\begin{array}{l}-0.00330 \\
(0.00247)\end{array}$ & $\begin{array}{l}0.00956^{* * * *} \\
(0.00224)\end{array}$ & $\begin{array}{c}0.00858^{* * *} \\
(0.00218)\end{array}$ & $\begin{array}{c}0.00826^{* * *} \\
(0.00235)\end{array}$ \\
\hline Airports & $\begin{array}{c}0.96286^{* * *} \\
(0.16796)\end{array}$ & $\begin{array}{l}1.29091^{* * * *} \\
(0.26370)\end{array}$ & $\begin{array}{l}2.52004^{* * * *} \\
(0.45371)\end{array}$ & $\begin{array}{c}0.83359^{* * * *} \\
(0.15064)\end{array}$ & $\begin{array}{l}1.05955^{* * * *} \\
(0.24058)\end{array}$ & $\begin{array}{c}2.06885^{* * * *} \\
(0.40802)\end{array}$ \\
\hline Mining Sites & $\begin{array}{l}1.33839^{*} \\
(0.78980)\end{array}$ & $\begin{array}{l}-2.12201^{* *} \\
(0.82385)\end{array}$ & $\begin{array}{l}-4.44490 \\
(3.38357)\end{array}$ & $\begin{array}{c}0.94847 \\
(0.72437)\end{array}$ & $\begin{array}{c}-1.98507^{* * *} \\
(0.72174)\end{array}$ & $\begin{array}{l}-4.12338 \\
(2.93097)\end{array}$ \\
\hline Road Length & $\begin{array}{c}-0.00121^{* * *} \\
(0.00028)\end{array}$ & $\begin{array}{l}-0.00002 \\
(0.00045)\end{array}$ & $\begin{array}{l}-0.00008 \\
(0.00067)\end{array}$ & $\begin{array}{c}-0.00110^{* * *} \\
(0.00026)\end{array}$ & $\begin{array}{l}-0.00000 \\
(0.00041)\end{array}$ & $\begin{array}{c}0.00001 \\
(0.00060)\end{array}$ \\
\hline Education of $\mathrm{HHH}$ & & & & $\begin{array}{c}0.15930^{* * * *} \\
(0.01151) \\
\end{array}$ & $\begin{array}{c}0.14834^{* * *} \\
(0.01143) \\
\end{array}$ & $\begin{array}{c}0.14580^{* * * *} \\
(0.01180) \\
\end{array}$ \\
\hline $\begin{array}{l}\text { Distance Radius } \\
\text { Households }\end{array}$ & $\begin{array}{c}200 \mathrm{~km} \\
8435\end{array}$ & $\begin{array}{c}100 \mathrm{~km} \\
8435\end{array}$ & $\begin{array}{c}50 \mathrm{~km} \\
8435\end{array}$ & $\begin{array}{c}200 \mathrm{~km} \\
8435\end{array}$ & $\begin{array}{c}100 \mathrm{~km} \\
8435\end{array}$ & $\begin{array}{c}50 \mathrm{~km} \\
8435\end{array}$ \\
\hline Excluded instruments & 48.12 & 38.04 & 19.32 & 46.87 & 36.23 & 18.48 \\
\hline 2nd Stage F statistic & 62.00 & 64.98 & 63.79 & 74.69 & 79.38 & 80.44 \\
\hline Latitude (1st Stage) & $\begin{array}{c}7.639498^{* * *} \\
(1.190066)\end{array}$ & $\begin{array}{c}3.211406^{* * * *} \\
(.61554)\end{array}$ & $\begin{array}{l}1.329128^{* * * *} \\
(.3070134)\end{array}$ & $\begin{array}{l}7.582759^{* * * *} \\
(1.187402)\end{array}$ & $\begin{array}{c}3.127866^{* * * *} \\
(.6118135)\end{array}$ & $\begin{array}{l}1.296465^{* * * *} \\
(.3038904)\end{array}$ \\
\hline Longitude (1st Stage) & $\begin{array}{l}5.435418^{* * * *} \\
(1.070676)\end{array}$ & $\begin{array}{c}3.950253^{* * * *} \\
(.6271242)\end{array}$ & $\begin{array}{l}1.197047^{* * * *} \\
(.2820365)\end{array}$ & $\begin{array}{c}5.367359^{* * *} \\
(1.076918)\end{array}$ & $\begin{array}{c}3.865526^{* * * *} \\
(.6299652)\end{array}$ & $\begin{array}{l}1.163416^{* * * * *} \\
(.2831917)\end{array}$ \\
\hline Hansen $\mathbf{J}$ - P-value & 0.8078 & 0.9172 & 0.5953 & 0.9388 & 0.9726 & 0.5885 \\
\hline
\end{tabular}




\subsubsection{Different Measure of Conflict Exposure}

In addition to differentiating measures of conflict exposure on the basis of three different distances $(200 \mathrm{~km}, 100 \mathrm{~km}$, and $50 \mathrm{~km})$, in this section I split measures of conflict exposure along temporal dimensions. Since there are distinct periods of conflict in the DRC (the first and second civil wars and current conflict events in the eastern provinces), I explore how different conflict periods matter for durable ownership and living conditions. Defined here, the split between the two conflicts takes place on January 1, 2004, with all conflict events in the DRC taking place on or after this day referred to as conflict events during the 'Kivu crisis' and all events taking place before this day referred to as the first and second civil war period. In structuring the specifications in such a way, I can better understand the different effects of conflict exposure at different times of the conflict.

Another way I check the robustness of the baseline results is to differentiate conflict exposure on the basis of different conflict events. The ACLED project categorizes each conflict event as being (1) a battle in which the government regains territory, (2) a battle in which there is no change of territory, (3) a battle in which rebels overtake territory, (4) the establishment of a rebel headquarters or base, (5) non-violent activity by a conflict actor, (6) a non-violent transfer of territory, (7) riots or protests, and (8) violence against civilians. I explore whether differentiating these conflict events on the basis of civilian impact has an influence on the results. To do so, I create a measure of civilian impact, where the variable is coded as a ' 1 ' if the conflict events pertain to situations $1,2,3,4$, or 8 . The other events receive a ' 0 '. This will test the robustness of the results by excluding conflict events that likely have less of an impact on the civilian population. 
The results of the robustness checks are presented in Table 2.12 and Table 2.13. Table 2.12 presents the results from constraining conflict exposure to those events taking place during the first and second civil wars that have had a civilian impact. The conflict exposure coefficients are negative, fairly large in magnitude, and vary in statistical significance. In two of the specifications, the correlations between conflict exposure and the latitude and longitude coordinate instruments are relatively low (though still highly statistically significant), which implies these may not be the strongest of instruments (i.e., the F test statistic is just less than 10). When I use the number of conflict events taking place after the beginning of 2004 for my measure of conflict exposure (with various distance radii), the coefficient estimates for conflict exposure are negative, large, and still vary in statistical significance on the basis of how conflict exposure is constructed. These results are also largely robust to differentiating the sample of conflict events for whether specific conflict events have had an impact on the civilian population. 
Table 2.12: Instrumental Variable Regression Results - ACLED Restricted

(1)

\begin{tabular}{|c|c|c|c|c|c|c|}
\hline & (1) & (2) & (3) & (4) & (5) & (6) \\
\hline Conflict & 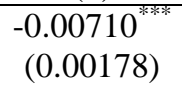 & $\begin{array}{c}-0.01446^{* * *} \\
(0.00374)\end{array}$ & $\begin{array}{c}-0.03477^{* * * *} \\
(0.01070)\end{array}$ & $\begin{array}{c}-0.00521^{* * * *} \\
(0.00160)\end{array}$ & $\begin{array}{c}-0.01033^{* * * *} \\
(0.00334)\end{array}$ & $\begin{array}{c}-0.02544^{* * * * *} \\
(0.00916)\end{array}$ \\
\hline HH Members & $\begin{array}{c}0.15133^{* * *} \\
(0.01328)\end{array}$ & $\begin{array}{c}0.14062^{* * * *} \\
(0.01360)\end{array}$ & $\begin{array}{c}0.13318^{* * *} \\
(0.01557)\end{array}$ & $\begin{array}{c}0.12896^{* * * *} \\
(0.01159)\end{array}$ & $\begin{array}{c}0.11981^{* * * *} \\
(0.01184)\end{array}$ & $\begin{array}{c}0.11421^{* * *} \\
(0.01327)\end{array}$ \\
\hline Capital or Large City & $\begin{array}{c}3.53009^{* * *} \\
(0.25805)\end{array}$ & $\begin{array}{c}3.22241^{* * *} \\
(0.35036)\end{array}$ & $\begin{array}{l}1.74006^{* * *} \\
(0.54907)\end{array}$ & $\begin{array}{c}2.87403^{* * *} \\
(0.23298)\end{array}$ & $\begin{array}{c}2.67022^{* * *} \\
(0.31557)\end{array}$ & $\begin{array}{l}1.44644^{* * *} \\
(0.47498)\end{array}$ \\
\hline Small City & $\begin{array}{c}2.54568^{* * * *} \\
(0.88311)\end{array}$ & $\begin{array}{c}2.90657^{* * *} \\
(0.75899)\end{array}$ & $\begin{array}{c}2.49391^{* * *} \\
(0.74387)\end{array}$ & $\begin{array}{c}2.21125^{* * *} \\
(0.75064)\end{array}$ & $\begin{array}{c}2.57310^{* * * *} \\
(0.66625)\end{array}$ & $\begin{array}{c}2.23944^{* * *} \\
(0.66281)\end{array}$ \\
\hline Town & $\begin{array}{l}1.73327^{* * *} \\
(0.28138)\end{array}$ & $\begin{array}{c}1.74408^{* * *} \\
(0.31681)\end{array}$ & $\begin{array}{l}1.97916^{* * *} \\
(0.37884)\end{array}$ & $\begin{array}{l}1.36251^{* * * *} \\
(0.25892)\end{array}$ & $\begin{array}{l}1.41125^{* * * *} \\
(0.28628)\end{array}$ & $\begin{array}{l}1.59431^{* * *} \\
(0.32617)\end{array}$ \\
\hline Altitude (log) & $\begin{array}{c}0.28072^{*} \\
(0.16761)\end{array}$ & $\begin{array}{c}0.23177 \\
(0.17152)\end{array}$ & $\begin{array}{l}0.37543^{* *} \\
(0.18597)\end{array}$ & $\begin{array}{c}0.26606^{*} \\
(0.15222)\end{array}$ & $\begin{array}{c}0.21322 \\
(0.15935)\end{array}$ & $\begin{array}{c}0.33268^{*} \\
(0.17002)\end{array}$ \\
\hline Under 5 Fraction & $\begin{array}{c}-1.33821^{* * * *} \\
(0.19260)\end{array}$ & $\begin{array}{c}-1.340566^{* * *} \\
(0.20580)\end{array}$ & $\begin{array}{c}-1.34184^{* * * *} \\
(0.21740)\end{array}$ & $\begin{array}{c}-1.12214^{* * * *} \\
(0.17706)\end{array}$ & $\begin{array}{c}-1.13908^{* * * *} \\
(0.18900)\end{array}$ & $\begin{array}{c}-1.14335^{* * * *} \\
(0.19539)\end{array}$ \\
\hline Male $\mathrm{HHH}$ & $\begin{array}{c}0.70728^{* * *} \\
(0.07279)\end{array}$ & $\begin{array}{c}0.71449^{* * *} \\
(0.07311)\end{array}$ & $\begin{array}{c}0.74595^{* * *} \\
(0.07336)\end{array}$ & $\begin{array}{c}0.13933^{*} \\
(0.07900)\end{array}$ & $\begin{array}{l}0.18283^{* *} \\
(0.07997)\end{array}$ & $\begin{array}{c}0.22529^{* * *} \\
(0.08133)\end{array}$ \\
\hline Age of $\mathrm{HHH}$ & $\begin{array}{c}-0.00252 \\
(0.00222)\end{array}$ & $\begin{array}{c}-0.00293 \\
(0.00221)\end{array}$ & $\begin{array}{c}-0.00172 \\
(0.00248)\end{array}$ & $\begin{array}{c}0.01010^{* * * *} \\
(0.00220)\end{array}$ & $\begin{array}{c}0.00902^{* * *} \\
(0.00217)\end{array}$ & $\begin{array}{c}0.00947^{* * *} \\
(0.00231)\end{array}$ \\
\hline Airports & $\begin{array}{c}0.84216^{* * *} \\
(0.14967)\end{array}$ & $\begin{array}{l}1.05906^{* * *} \\
(0.23985)\end{array}$ & $\begin{array}{l}2.62914^{* * *} \\
(0.49759)\end{array}$ & $\begin{array}{c}0.74317^{* * *} \\
(0.13375)\end{array}$ & $\begin{array}{c}0.88824^{* * * *} \\
(0.21507)\end{array}$ & $\begin{array}{c}2.14908^{* * *} \\
(0.44244)\end{array}$ \\
\hline Mining Sites & $\begin{array}{c}0.56338 \\
(0.67235)\end{array}$ & $\begin{array}{c}-2.25896^{* * *} \\
(0.84503)\end{array}$ & $\begin{array}{c}-7.33489^{* * *} \\
(3.36067)\end{array}$ & $\begin{array}{c}0.37362 \\
(0.60718)\end{array}$ & $\begin{array}{c}-2.08037^{* * * *} \\
(0.73761)\end{array}$ & $\begin{array}{l}-6.23493^{* * *} \\
(2.92284)\end{array}$ \\
\hline Road Length & $\begin{array}{c}-0.00104^{* * *} \\
(0.00025)\end{array}$ & $\begin{array}{c}0.00024 \\
(0.00046)\end{array}$ & $\begin{array}{c}0.00020 \\
(0.00072)\end{array}$ & $\begin{array}{c}-0.00097^{* * *} \\
(0.00023)\end{array}$ & $\begin{array}{c}0.00018 \\
(0.00042)\end{array}$ & $\begin{array}{c}0.00022 \\
(0.00064)\end{array}$ \\
\hline Education of $\mathrm{HHH}$ & & & & $\begin{array}{c}0.16130^{* * *} \\
(0.01130)\end{array}$ & $\begin{array}{c}0.15160^{* * *} \\
(0.01126)\end{array}$ & $\begin{array}{c}0.14659^{* * *} \\
(0.01185)\end{array}$ \\
\hline $\begin{array}{l}\text { Distance Radius } \\
\mathrm{N}\end{array}$ & $\begin{array}{l}200 \mathrm{~km} \\
8435\end{array}$ & $\begin{array}{c}100 \mathrm{~km} \\
8435\end{array}$ & $\begin{array}{c}50 \mathrm{~km} \\
8435\end{array}$ & $\begin{array}{c}200 \mathrm{~km} \\
8435\end{array}$ & $\begin{array}{c}100 \mathrm{~km} \\
8435\end{array}$ & $\begin{array}{c}50 \mathrm{~km} \\
8435\end{array}$ \\
\hline Excluded instruments & $\begin{array}{l}40.76 \\
61.77\end{array}$ & 26.60 & 9.68 & 40.42 & 25.96 & 9.52 \\
\hline $\begin{array}{l}\text { 2nd Stage F statistic } \\
\text { Latitude (1st Stage) }\end{array}$ & $\begin{array}{c}61.77 \\
10.63107^{* * * *}\end{array}$ & $\begin{array}{l}54.02 \\
4.393028^{* * * *} \\
(8610038)\end{array}$ & $\begin{array}{l}58.40 \\
1.775085^{* * *}\end{array}$ & $\begin{array}{l}76.17 \\
10.58889^{* * * *}\end{array}$ & $\begin{aligned} 71.02 \\
4.327561^{* * * *} \\
(8586346)\end{aligned}$ & $\begin{aligned} & 76.43 \\
1.731687^{* * *} & (5102089)\end{aligned}$ \\
\hline Longitude (1st Stage) & $\begin{array}{l}7.939947^{* * * *} \\
(1.42195)\end{array}$ & $\begin{array}{l}4.535423^{* * *} \\
(.7412775)\end{array}$ & $\begin{array}{l}1.900361^{* * * *} \\
(.5030431)\end{array}$ & $\begin{array}{l}7.889344^{* * * *} \\
(1.424381)\end{array}$ & $\begin{array}{l}4.469026^{* * * *} \\
(.7400614)\end{array}$ & $\begin{array}{l}1.855678^{* * * *} \\
(.4980616)\end{array}$ \\
\hline Hansen $\mathbf{J}$ - P-value & 0.8541 & 0.8960 & 0.7726 & 0.9809 & 0.8784 & 0.7335 \\
\hline
\end{tabular}

Clustered standard errors (clustered at the DHS cluster location) in parentheses. Statistical significance is indicated as follows: ${ }^{*} p<0.10,{ }^{* *} p<0.05,{ }^{* * * *} p<0.01$. The dependent variable is the original DHS wealth index. Specifications (1) and (3) use a measure of conflict exposure that counts all conflict events that have taken place within a $200 \mathrm{~km}$ radius. Specifications (2) and (4) are for $100 \mathrm{~km}$ radii and Specifications (3) and (6) $50 \mathrm{~km}$ radii. A constant term is included in each specification but is not reported. 
Table 2.13: Instrumental Variable Regression Results - ACLED Restricted

(2)

\begin{tabular}{|c|c|c|c|c|c|c|}
\hline & (1) & (2) & (3) & (4) & (5) & (6) \\
\hline Conflict & 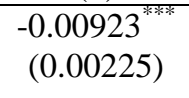 & $\begin{array}{c}-0.01596^{* * * *} \\
(0.00376)\end{array}$ & $\begin{array}{c}-0.04140 \\
(0.01035)\end{array}$ & $\begin{array}{c}-0.00680^{* * * *} \\
(0.00206)\end{array}$ & $\begin{array}{c}-0.01158^{* * * *} \\
(0.00349)\end{array}$ & $\begin{array}{c}-0.03066^{* * *} \\
(0.00962)\end{array}$ \\
\hline HH Members & $\begin{array}{c}0.14872^{* * *} \\
(0.01357)\end{array}$ & $\begin{array}{c}0.13420^{* * *} \\
(0.01352)\end{array}$ & $\begin{array}{c}0.13043^{* * *} \\
(0.01370)\end{array}$ & $\begin{array}{c}0.12728^{* * * *} \\
(0.01183)\end{array}$ & $\begin{array}{c}0.11593^{* * *} \\
(0.01185)\end{array}$ & $\begin{array}{c}0.11294^{* * *} \\
(0.01207)\end{array}$ \\
\hline Capital or Large City & $\begin{array}{c}3.39292^{* * *} \\
(0.28253)\end{array}$ & $\begin{array}{c}3.02719^{* * *} \\
(0.37785)\end{array}$ & $\begin{array}{l}1.94398^{* * *} \\
(0.54227)\end{array}$ & $\begin{array}{c}2.78011^{* * *} \\
(0.24944)\end{array}$ & $\begin{array}{c}2.54875^{* * *} \\
(0.33353)\end{array}$ & $\begin{array}{l}1.61145^{* * *} \\
(0.46500)\end{array}$ \\
\hline Small City & $\begin{array}{c}2.50167^{* * * *} \\
(0.93382)\end{array}$ & $\begin{array}{c}2.91726^{* * *} \\
(0.75597)\end{array}$ & $\begin{array}{c}2.58561^{* * *} \\
(0.73319)\end{array}$ & $\begin{array}{c}2.18265^{* * *} \\
(0.78600)\end{array}$ & $\begin{array}{c}2.59283^{* * *} \\
(0.66379)\end{array}$ & $\begin{array}{c}2.31924^{* * *} \\
(0.65350)\end{array}$ \\
\hline Town & $\begin{array}{l}1.59311^{* * *} \\
(0.28350)\end{array}$ & $\begin{array}{l}1.70630^{* * *} \\
(0.30020)\end{array}$ & $\begin{array}{l}1.72399^{* * *} \\
(0.32617)\end{array}$ & $\begin{array}{l}1.26330^{* * * *} \\
(0.25849)\end{array}$ & $\begin{array}{l}1.39605^{* * * *} \\
(0.27674)\end{array}$ & $\begin{array}{l}1.42073^{* * *} \\
(0.29302)\end{array}$ \\
\hline Altitude (log) & $\begin{array}{l}0.48244^{* *} \\
(0.20958)\end{array}$ & $\begin{array}{l}0.43175^{* *} \\
(0.21483)\end{array}$ & $\begin{array}{l}0.50858^{* *} \\
(0.22282)\end{array}$ & $\begin{array}{l}0.41459^{* * *} \\
(0.18431)\end{array}$ & $\begin{array}{c}0.35924^{*} \\
(0.19273)\end{array}$ & $\begin{array}{l}0.43220^{* *} \\
(0.19918)\end{array}$ \\
\hline Under 5 Fraction & $\begin{array}{c}-1.27322^{* * *} \\
(0.19403)\end{array}$ & $\begin{array}{c}-1.22073^{* * *} \\
(0.19016)\end{array}$ & $\begin{array}{c}-1.21882^{* * * *} \\
(0.19523)\end{array}$ & $\begin{array}{c}-1.07655^{* * * *} \\
(0.17757)\end{array}$ & $\begin{array}{c}-1.05969^{* * * *} \\
(0.17743)\end{array}$ & $\begin{array}{c}-1.05989^{* * * *} \\
(0.17908)\end{array}$ \\
\hline Male $\mathrm{HHH}$ & $\begin{array}{c}0.65937^{* * *} \\
(0.07657)\end{array}$ & $\begin{array}{c}0.69195^{* * *} \\
(0.07333)\end{array}$ & $\begin{array}{c}0.69955^{* * *} \\
(0.07379)\end{array}$ & $\begin{array}{c}0.11008 \\
(0.08007)\end{array}$ & $\begin{array}{l}0.18615^{* *} \\
(0.08009)\end{array}$ & $\begin{array}{c}0.21189^{* * *} \\
(0.07990)\end{array}$ \\
\hline Age of $\mathrm{HHH}$ & $\begin{array}{c}-0.00212 \\
(0.00227)\end{array}$ & $\begin{array}{c}-0.00284 \\
(0.00220)\end{array}$ & $\begin{array}{l}-0.00293 \\
(0.00246)\end{array}$ & $\begin{array}{l}0.01026^{* * * *} \\
(0.00224)\end{array}$ & $\begin{array}{c}0.00864^{* * *} \\
(0.00218)\end{array}$ & $\begin{array}{c}0.00813^{* * *} \\
(0.00236)\end{array}$ \\
\hline Airports & $\begin{array}{c}0.97692^{* * *} \\
(0.17225)\end{array}$ & $\begin{array}{l}1.16485^{* * *} \\
(0.24872)\end{array}$ & $\begin{array}{l}1.97697^{* * *} \\
(0.36933)\end{array}$ & $\begin{array}{c}0.84329^{* * * *} \\
(0.15421)\end{array}$ & $\begin{array}{c}0.97164^{* * *} \\
(0.22666)\end{array}$ & $\begin{array}{l}1.68250^{* * * *} \\
(0.33327)\end{array}$ \\
\hline Mining Sites & $\begin{array}{c}0.86508 \\
(0.71509)\end{array}$ & $\begin{array}{c}-2.38890^{* * * *} \\
(0.78658)\end{array}$ & $\begin{array}{l}-1.87166 \\
(3.04487)\end{array}$ & $\begin{array}{c}0.59712 \\
(0.64962)\end{array}$ & $\begin{array}{c}-2.18098^{* * * *} \\
(0.69852)\end{array}$ & $\begin{array}{c}-2.22846 \\
(2.70267)\end{array}$ \\
\hline Road Length & $\begin{array}{c}-0.00129^{* * *} \\
(0.00030)\end{array}$ & $\begin{array}{l}-0.00005 \\
(0.00046)\end{array}$ & $\begin{array}{c}0.00042 \\
(0.00072)\end{array}$ & $\begin{array}{c}-0.00116^{* * *} \\
(0.00027)\end{array}$ & $\begin{array}{c}-0.00002 \\
(0.00041)\end{array}$ & $\begin{array}{c}0.00038 \\
(0.00065)\end{array}$ \\
\hline Education of $\mathrm{HHH}$ & & & & $\begin{array}{c}0.15959^{* * *} \\
(0.01168)\end{array}$ & $\begin{array}{c}0.14599^{* * *} \\
(0.01191)\end{array}$ & $\begin{array}{c}0.14067^{* * *} \\
(0.01259)\end{array}$ \\
\hline Distance Radius & $200 \mathrm{~km}$ & $100 \mathrm{~km}$ & $50 \mathrm{~km}$ & $200 \mathrm{~km}$ & $100 \mathrm{~km}$ & $50 \mathrm{~km}$ \\
\hline Households & 8435 & 8435 & 8435 & 8435 & 8435 & 8435 \\
\hline Excluded instruments & 39.23 & 33.21 & 24.07 & 37.96 & 31.05 & 22.40 \\
\hline 2nd Stage F statistic & 60.90 & 63.69 & 67.22 & 74.67 & 77.89 & 83.57 \\
\hline Latitude (1st Stage) & $\begin{array}{l}8.087064^{* * * *} \\
(1.359117)\end{array}$ & $\begin{array}{c}3.662926^{* * *} \\
(.6692769)\end{array}$ & $\begin{array}{l}1.620142^{* * *} \\
(1.620142)\end{array}$ & $\begin{array}{l}8.030116^{* * *} \\
(1.361984)\end{array}$ & $\begin{array}{c}3.545897^{* * *} \\
(.6661464)\end{array}$ & $\begin{array}{l}1.561324^{* * *} \\
(.3023823)\end{array}$ \\
\hline Longitude (1st Stage) & $\begin{array}{c}6.201769^{* * * *} \\
(1.322377)\end{array}$ & $\begin{array}{c}4.348495^{* * * *} \\
(.8226885)\end{array}$ & $\begin{array}{l}1.475403^{* * *} \\
(.3216525)\end{array}$ & $\begin{array}{l}6.13346^{* * *} \\
(1.328749)\end{array}$ & $\begin{array}{c}4.229803^{* * *} \\
(.8279428)\end{array}$ & $\begin{array}{c}1.414843^{* * *} \\
(.321932)\end{array}$ \\
\hline Hansen $\mathbf{J}$ - P-value & 0.8849 & 0.9555 & 0.6011 & 0.9983 & 0.9981 & 0.5953 \\
\hline
\end{tabular}

Clustered standard errors (clustered at the DHS cluster location) in parentheses. Statistical significance is indicated as follows: ${ }^{*} p<0.10,{ }^{* *} p<0.05,{ }^{* * * *} p<0.01$. The dependent variable is the original DHS wealth index. Specifications (1) and (3) use a measure of conflict exposure that counts all conflict events that have taken place within a $200 \mathrm{~km}$ radius. Specifications (2) and (4) are for $100 \mathrm{~km}$ radii and Specifications (3) and (6) $50 \mathrm{~km}$ radii. A constant term is included in each specification but is not reported. 


\subsubsection{Distance from Kinshasa}

One additional way to test the robustness of the results is to include distance from Kinshasa as a control variable. It is plausible that distance from Kinshasa could influence household-level economic conditions because (a) wealth is simply less further from the capital, perhaps because of the historical distribution of spending on public services or because of a withdrawal of support during the latter stages of the Mobutu regime or (b) the government does not exercise territorial control over the eastern and northeastern provinces of the country and, as such, the levels of conflict will be more. I include this variable in the regression analyses. These results are presented in Table 2.14. For some of the specifications, the coefficient estimate for conflict exposure is no longer statistically significant, though it remains so when using the distance measures of $100 \mathrm{~km}$ and $50 \mathrm{~km}$. I don't find much evidence of a strong impact on householdlevel wealth of being far or close to Kinshasa, perhaps because I control for a number of clusterlevel factors that influence wealth (like the locations of roads and airports). 
Table 2.14: Instrumental Variable Regression Results Using Distance from Kinshasa as a Covariate

\begin{tabular}{|c|c|c|c|c|c|c|}
\hline & $(1)$ & (2) & (3) & $(4)$ & $(5)$ & (6) \\
\hline Conflict & $\begin{array}{c}-0.00169 \\
(0.00113)\end{array}$ & $\begin{array}{c}-0.00699^{* *} \\
(0.00344)\end{array}$ & $\begin{array}{l}-0.01661^{* *} \\
(0.00700)\end{array}$ & $\begin{array}{c}-0.00153 \\
(0.00105)\end{array}$ & $\begin{array}{l}-0.00627^{*} \\
(0.00329)\end{array}$ & $\begin{array}{c}-0.01517^{* *} \\
(0.00677)\end{array}$ \\
\hline HH Members & $\begin{array}{c}0.14959^{* * * *} \\
(0.01234)\end{array}$ & $\begin{array}{c}0.13830^{* * *} \\
(0.01353)\end{array}$ & $\begin{array}{c}0.13402^{* * *} \\
(0.01433)\end{array}$ & $\begin{array}{c}0.12762^{* * *} \\
(0.01113)\end{array}$ & $\begin{array}{c}0.11816^{* * *} \\
(0.01222)\end{array}$ & $\begin{array}{c}0.11454^{* * *} \\
(0.01314)\end{array}$ \\
\hline Capital or Large City & $\begin{array}{c}3.53579^{* * *} \\
(0.23686)\end{array}$ & $\begin{array}{c}3.18270^{* * * *} \\
(0.39174)\end{array}$ & $\begin{array}{l}1.81012^{* * *} \\
(0.68356)\end{array}$ & $\begin{array}{c}2.87200^{* * * *} \\
(0.22252)\end{array}$ & $\begin{array}{c}2.62264^{* * *} \\
(0.36319)\end{array}$ & $\begin{array}{l}1.33739^{* *} \\
(0.62768)\end{array}$ \\
\hline Small City & $\begin{array}{c}2.79853^{* * *} \\
(0.82051)\end{array}$ & $\begin{array}{c}2.87532^{* * *} \\
(0.76722)\end{array}$ & $\begin{array}{c}2.46658^{* * *} \\
(0.77830)\end{array}$ & $\begin{array}{c}2.34853^{* * * *} \\
(0.71527)\end{array}$ & $\begin{array}{c}2.49141^{\text {*** }} \\
(0.68607)\end{array}$ & $\begin{array}{c}2.10665^{* * *} \\
(0.71955)\end{array}$ \\
\hline Town & $\begin{array}{l}1.77170^{* * *} \\
(0.25023)\end{array}$ & $\begin{array}{l}1.70255^{* * *} \\
(0.30616)\end{array}$ & $\begin{array}{l}1.84462^{* * *} \\
(0.33828)\end{array}$ & $\begin{array}{l}1.37124^{* * *} \\
(0.23884)\end{array}$ & $\begin{array}{l}1.35294^{* * *} \\
(0.29237)\end{array}$ & $\begin{array}{l}1.48906^{* * *} \\
(0.32200)\end{array}$ \\
\hline Altitude (log) & $\begin{array}{l}0.31532^{* *} \\
(0.15355)\end{array}$ & $\begin{array}{c}0.32590^{*} \\
(0.18608)\end{array}$ & $\begin{array}{l}0.42706^{* * *} \\
(0.19431)\end{array}$ & $\begin{array}{l}0.29333^{* *} \\
(0.14640)\end{array}$ & $\begin{array}{c}0.29327 \\
(0.17998)\end{array}$ & $\begin{array}{l}0.39523^{* *} \\
(0.18653)\end{array}$ \\
\hline Under 5 Fraction & $\begin{array}{c}-1.28652^{* * *} \\
(0.18155)\end{array}$ & $\begin{array}{c}-1.30629^{* * *} \\
(0.20107)\end{array}$ & $\begin{array}{c}-1.31308^{* * *} \\
(0.20731)\end{array}$ & $\begin{array}{c}-1.08830^{* * *} \\
(0.17135)\end{array}$ & $\begin{array}{c}-1.13136^{* * *} \\
(0.19413)\end{array}$ & $\begin{array}{c}-1.14265^{* * *} \\
(0.19922)\end{array}$ \\
\hline Male HHH & $\begin{array}{c}0.70402^{* * *} \\
(0.06936)\end{array}$ & $\begin{array}{c}0.70528^{* * *} \\
(0.07373)\end{array}$ & $\begin{array}{c}0.72704^{* * *} \\
(0.07292)\end{array}$ & $\begin{array}{c}0.13118^{*} \\
(0.07788)\end{array}$ & $\begin{array}{l}0.18706^{* * *} \\
(0.08206)\end{array}$ & $\begin{array}{c}0.22124^{* * *} \\
(0.08505)\end{array}$ \\
\hline Age of $\mathrm{HHH}$ & $\begin{array}{c}-0.00241 \\
(0.00208)\end{array}$ & $\begin{array}{l}-0.00297 \\
(0.00222)\end{array}$ & $\begin{array}{l}-0.00238 \\
(0.00250)\end{array}$ & $\begin{array}{c}0.01027^{* * * *} \\
(0.00215)\end{array}$ & $\begin{array}{c}0.00857^{* * * *} \\
(0.00231)\end{array}$ & $\begin{array}{c}0.00878^{* * *} \\
(0.00247)\end{array}$ \\
\hline Airports & $\begin{array}{c}0.66129^{* * *} \\
(0.21201)\end{array}$ & $\begin{array}{l}1.26808^{* * *} \\
(0.46956)\end{array}$ & $\begin{array}{c}2.78277^{* * *} \\
(0.86098)\end{array}$ & $\begin{array}{c}0.65996^{* * *} \\
(0.19960)\end{array}$ & $\begin{array}{l}1.18563^{* * *} \\
(0.44339)\end{array}$ & $\begin{array}{c}2.60355^{* * *} \\
(0.82066)\end{array}$ \\
\hline Mining Sites & $\begin{array}{c}0.17386 \\
(0.65056)\end{array}$ & $\begin{array}{c}-2.30636^{* * *} \\
(0.86343)\end{array}$ & $\begin{array}{l}-4.88065 \\
(3.32560)\end{array}$ & $\begin{array}{c}0.17084 \\
(0.61863)\end{array}$ & $\begin{array}{c}-2.20917^{* * * *} \\
(0.79136)\end{array}$ & $\begin{array}{l}-4.61123 \\
(3.08131)\end{array}$ \\
\hline Road Length & $\begin{array}{c}-0.00086^{* * *} \\
(0.00028)\end{array}$ & $\begin{array}{c}-0.00001 \\
(0.00046)\end{array}$ & $\begin{array}{c}-0.00004 \\
(0.00071)\end{array}$ & $\begin{array}{c}-0.00089^{* * * *} \\
(0.00026)\end{array}$ & $\begin{array}{c}0.00003 \\
(0.00043)\end{array}$ & $\begin{array}{c}0.00006 \\
(0.00067)\end{array}$ \\
\hline Distance from Kinshasa & $\begin{array}{l}-0.00055^{*} \\
(0.00028)\end{array}$ & $\begin{array}{l}-0.00009 \\
(0.00039)\end{array}$ & $\begin{array}{c}-0.00024 \\
(0.00029)\end{array}$ & $\begin{array}{c}-0.00032 \\
(0.00025)\end{array}$ & $\begin{array}{c}0.00008 \\
(0.00035)\end{array}$ & $\begin{array}{l}-0.00006 \\
(0.00027)\end{array}$ \\
\hline Education of $\mathrm{HHH}$ & & & & $\begin{array}{c}0.16214^{* * * *} \\
(0.01119) \\
\end{array}$ & $\begin{array}{c}0.14743^{* * *} \\
(0.01273) \\
\end{array}$ & $\begin{array}{c}0.14337^{* * *} \\
(0.01356) \\
\end{array}$ \\
\hline $\begin{array}{l}\text { Distance Radius } \\
\mathrm{N}\end{array}$ & $\begin{array}{c}200 \mathrm{~km} \\
8435\end{array}$ & $\begin{array}{c}100 \mathrm{~km} \\
8435\end{array}$ & $\begin{array}{c}50 \mathrm{~km} \\
8435\end{array}$ & $\begin{array}{c}200 \mathrm{~km} \\
8435\end{array}$ & $\begin{array}{c}100 \mathrm{~km} \\
8435\end{array}$ & $\begin{array}{c}50 \mathrm{~km} \\
8435\end{array}$ \\
\hline Excluded Instruments & 41.47 & 21.05 & 17.10 & 41.65 & 21.25 & 17.42 \\
\hline 2nd Stage F statistic & 64.15 & 52.59 & 55.55 & 76.07 & 61.93 & 68.05 \\
\hline Latitude (1st Stage) & $\begin{array}{c}23.34538^{* * * *} \\
(2.90833)\end{array}$ & $\begin{array}{l}9.262956^{* * *} \\
(1.528895)\end{array}$ & $\begin{array}{l}3.912556^{* * * *} \\
(.7573367)\end{array}$ & $\begin{array}{l}23.20865^{* * *} \\
(2.905465)\end{array}$ & $\begin{array}{l}9.114295^{* * *} \\
(1.512115)\end{array}$ & $\begin{array}{c}3.836067^{* * * *} \\
(.7451316)\end{array}$ \\
\hline Longitude (1st Stage) & $\begin{array}{l}56.5922^{* * *} \\
(9.652096)\end{array}$ & $\begin{array}{l}15.65976^{* * * *} \\
(4.835392)\end{array}$ & $\begin{array}{l}7.155374^{* * * *} \\
(2.667474)\end{array}$ & $\begin{array}{l}56.90603^{* * *} \\
(9.613572) \\
\end{array}$ & $\begin{array}{l}15.95835^{* * * *} \\
(4.778126)\end{array}$ & $\begin{array}{l}7.292282^{* * * *} \\
(2.640511)\end{array}$ \\
\hline Hansen J-P-value & 0.1165 & 0.9876 & 0.8239 & 0.2533 & 0.6129 & 0.5029 \\
\hline
\end{tabular}

Notes: See above table. 


\subsection{Conclusion and Discussion}

Civil war is 'development in reverse' (Collier et al., 2003) and nowhere has this been more evident than in the Democratic Republic of the Congo. The evidence put forth in this paper suggests that the conflict events that have taken place across the DRC have had a significant negative impact on household-level durable ownership and living conditions. ${ }^{31}$ This finding is consistent with many of the qualitative reports on the topic and gives an empirical contribution to the literature. More broadly, the results illustrate how local political incentives and political agendas generate significant economic externalities for the population at large.

While the results are fairly intuitive, there are a number of limitations to this study that should be noted. First, the instrumental variable strategy employed in this paper makes the assumption that the instruments (the distances between DHS cluster locations and Goma, and the latitude and longitude coordinates of the DHS cluster locations) only influence household-level economic conditions through their impact on cluster-level conflict exposure. It is plausible, however, that other historical or economic factors can also affect economic conditions through this distance channel. Second, while the data used in this paper are novel for the DRC, they are still measured with an amount of imprecision that should be considered. Lastly, more research must be conducted on the role of the mineral sector in affecting the location of violence across the country. The findings regarding the role of the mineral sector in influencing both conflict events and household-level economic conditions should be viewed as suggestive rather than

\footnotetext{
31 This finding highlights the economic consequence of civil war in the DRC, yet this is only one of the consequences. As described by Autesserre (2010: 2), as a result of civil war, "an estimated one thousand civilians die every day, mostly due to malnutrition and diseases that could be easily prevented if the Congo's already weak economic and social structures had not collapsed during the conflict...81\% [of those living in the eastern provinces] had to flee their homes, more than half experienced the violent death of family members or friends, more than a third were abducted for at least a week, and $16 \%$ were subject to sexual violence, usually repeatedly."
} 
definitive. Ultimately, until more data are available for the DRC, there are still a number of things we simply do not know about the causes and consequences of conflict across the country.

Generally, the results of this paper suggest there are two important areas of research that must be better developed. First, while differences in meta-level institutional and political factors partly explain cross-country variation in economic performance (see, e.g., Acemoglu, Johnson, and Robinson, 2001), local political economy factors (e.g. the decisions and appropriative actions of rebel groups, government troops, etc.) also have strong implications for local economic development. Development economists must turn their attention towards understanding the local political processes by which economic outcomes are influenced. ${ }^{32}$ Second, more research is needed on the topics of conflict resolution, state building, and postconflict economic reconstruction, especially in the DRC. To date, only a handful of studies have addressed the political economy of these processes. Coyne (2008) analyzes the political economy of state-building efforts, highlighting how issues of local incentives and local knowledge will prevent most externally-planned state-building efforts from being successful. Autesserre (2010) assesses why international peace-building efforts in the DRC have largely been ineffective, pointing to a systematic lack of attention to local political economy issues. In essence, there are a number of constraints on what those involved in peace-building efforts can accomplish. Finding alternative mechanisms for conflict resolution is therefore crucial.

\footnotetext{
${ }^{32}$ To be sure, local political economy factors are likely to be related to national-level political institutions or regional protection and enforcement of property rights.
} 


\section{Chapter 3}

\section{Infant Mortality over the Electoral Cycle}

\subsection{Introduction}

Economists have long studied whether incumbent politicians 'electioneer' fiscal and monetary policy in order to improve their re-election prospects. Models by Rogoff and Sibert (1988) and Rogoff (1990) suggest that incumbent politicians will pursue policies that keep them in power and that these policies will often take the form of targeted transfer payments and highly visible government expenditures. As a real world example of this sort of electioneering, consider that in 2009 the opposition to the Ahmadinejad government in Iran argued that the government distributed 400,000 tons of potatoes around the country and gave bonuses (along with gold coins) to government workers in the run up to the presidential election (Fathi, 2009).

To date, the empirical literature on 'election-year economics' (Brender and Drazen, 2007) has mainly focused on whether there are electoral cycles in macro-level variables like employment, GDP, the money supply, tax rates, expenditures, and budget deficits. Evidence of opportunistic political business cycles is mixed (see recent studies by Grier and Grier, 2000; Drazen, 2001; Franzese, 2002; Brender and Drazen, 2007; Grier, 2008). There is much stronger empirical support for political budget cycles than political business cycles, though evidence seems to be limited to developing countries (Shi and Svensson, 2006), relatively young democracies (Brender and Drazen, 2005), and countries with relatively weak institutions 
(Schuknecht, 2000). ${ }^{33}$ Nevertheless, there has been recent evidence of electoral cyclicality in the OECD in public health expenditures (Potrafke, 2010).

This paper takes a different route than the existing literature by testing whether infant mortality rates fluctuate over the electoral cycle. Recent studies have shown that infant mortality rates across the developing world are highly sensitive to short-run fluctuations in aggregate income (Baird, Friedman, and Schady, 2011), weather patterns and disease ecology (Kudamatsu, Persson, and Stromberg, 2011), and commodity prices (Miller and Urdinola, 2010). In addition to these economic and environmental conditions, it is also important to assess whether there are important political economy explanations for fluctuations in infant mortality rates. Much of the literature on political budget cycles points to increases in transfer payments and deficit spending as the channels through which incumbent politicians seek to improve their chances of being reelected (Tufte, 1978; Rogoff, 1990; Schady, 2000), thus it is not unexpected to observe decreases in infant mortality prior to elections. To be certain, the impact of electoral cycles on infant mortality is not expected to be as large in magnitude as other economic shocks or humanitarian crises, yet there are reasons to believe the effects may be nontrivial.

Addressing whether there is electoral cyclicality in infant mortality is important for a number of reasons. First, Cutler, Deaton, and Lleras-Muney (2006) argue that diarrheal disease and respiratory infections - two of the main causes of infant and under-5 deaths in the developing world - are easily treated with oral rehydration therapy and antibiotics. The question remains why these treatments are not available to infants suffering from easily preventable illnesses, particularly among those countries that receive a large amount of health sector-specific aid. The answer likely rests with political economy explanations. To date, only a handful of studies have examined how specific political or institutional factors influence micro-level health

\footnotetext{
${ }^{33}$ See also Persson and Tabellini (2005).
} 
outcomes across the developing world. Kudamatsu (2011), for instance, presents evidence that democratization in Africa led to declines in infant mortality rates. Yet it is also important to assess whether there are political economy explanations for fluctuations in infant mortality, hence this paper. Second, studies on electoral cyclicality in fiscal policies and macroeconomic outcomes employ overly aggregated data on expenditures, tax rates, and other outcome measures (if they are available). This paper uses a rich source of information on individual level outcomes across the developing world, which allows me to control for infant- and mother-specific conditions that may affect the probability of infant mortality. Lastly, this question is interesting from an empirical perspective because the effects of elections on infant mortality are somewhat ambiguous. While transfers and government spending often increase prior to elections, so too do political instability, violence, and repression of opposition groups in countries with relatively weak institutions.

In examining election-year effects on infant mortality rates, I find only limited evidence of any sort of electoral cyclicality. In matching election dates with infant birth dates to create different exposure windows, I find some evidence that in utero exposure to presidential elections is associated with a reduced probability of infant mortality ex post, yet this result seems to be driven by the impact of democracy rather than elections per se. The results suggest that the level of electoral competition would need to be relatively high to observe declines in infant mortality rates prior to elections.

The paper proceeds as follows. I first discuss the potential transmission mechanisms by which electoral cycles may influence infant mortality rates in Section 2. Section 3 describes the data. Section 4 presents the empirical strategy. Section 5 presents the main results and discusses how different political institutions matter for the impact of elections on infant mortality rates. 
Section 6 examines differences in the timing of election dates and birth dates. Section 7 concludes.

\subsection{The Logic of Electoral Cycles}

In this section, I first discuss why elections provide incentives for incumbent politicians to pursue expansionary policies or increases in transfer payments prior to elections and what the literature suggests about the relationship between these transfer payments and infant health outcomes. I then discuss some of the other channels through which elections may influence infant health outcomes, with specific attention to pre- and post-electoral violence and political repression.

\subsubsection{Electoral Incentives and Infant Mortality}

An extensive literature on 'election economics' suggests that incumbent politicians pursue policies that increase their odds of being re-elected. ${ }^{34}$ Rogoff and Sibert (1988) and Rogoff (1990) develop models in which incumbent politicians seek to signal their competence to voters by increasing public spending and transfers and reducing taxes. Voters then support these incumbent politicians in their bid to stay in power because of the signals sent through the distribution of public goods and transfers. In Rogoff and Sibert (1988), electoral cycles in macroeconomic and fiscal policy variables are the result of temporary information asymmetries on the part of voters and incumbent politicians.

\footnotetext{
${ }^{34}$ The influence of electoral processes on the distribution of goods and services takes place in a variety of contexts, not only in developing countries but also in developed countries. Garrett and Sobel (2003), for instance, find that in the United States, states that were more important to the president electorally were more likely to be declared a federal disaster area. There were also more disasters declared during election years than in years without elections. Kubik and Moran (2003) find evidence for a gubernatorial election cycle in state executions: states have a much higher likelihood of executing death row inmates during gubernatorial election years than in other years.
} 
Rogoff (1990) develops a model in which incumbent politicians pursue 'quick-impact' policies-i.e. projects that are highly visible — as a means of improving their re-election prospects. As Rogoff (1990: 21) notes, “during election years, governments at all levels often engage in a consumption binge, in which taxes are cut, transfers are raised, and government spending is distorted toward projects with high immediate visibility." Electoral cycles often occur in tax rates, direct transfer payments (Tufte, 1978; Drazen, 2001; Bohn and Meon, 2009; Potrafke, 2010) or other in-kind transfers (as evinced in the case of the potato distribution in Iran in 2009).

These election-year expenditure changes may lead to short-run and long-run effects in infant mortality. Short-run effects would include direct transfers, the provision of in-kind nutritional supplements, potential improvements in government service provision, and other immediate benefits, while long-run effects would include the benefits of access to cleaner water, better sanitation facilities, and general improvements in infrastructure (in addition to potential increases in transparency and accountability due to the holding of elections).

Most neonatal and infant deaths in the developing world are easily preventable. These deaths are often proximately related to pregnancy and neonatal complications, infections, malaria exposure, and malnutrition. In many cases, maternal health also suffers as a result of malnutrition, poor prenatal care, and micronutrient deficiencies. Neonatal and infant mortality can be addressed through a series of relatively low cost preventative measures and interventions. ${ }^{35}$ Improvements in public health systems, sanitation facilities, infrastructure, the provision of in-kind nutritional supplements and other transfer payments (e.g., [un]conditional

\footnotetext{
${ }^{35}$ For instance, Kinney et al. (2010:2) argue that "many scientifically proven health interventions are available for maternal, newborn, and child health such as medicines, immunizations, insecticide-treated bed nets, and equipment for emergency obstetric care. Yet many African governments are currently underutilizing existing scientific knowledge to save women's and children's lives."
} 
cash transfer payments), and the expansion of pilot projects are some of the potential channels through which elections may be associated with reductions in infant mortality rates. Incumbent politicians may also want to increase the number or scope of donor-funded programs prior to an election (Desai and Kharas, 2010).

As noted by Sobel and Pellillo (2011), electoral incentives are often manifested in the behavior of the agents and bureaucrats that politicians oversee as well. Consider also that many neonatal deaths are related to poor access to maternal health and maternal and neonatal health services (Lawn et al., 2009). Because elections provide incentives for politicians to provide services for citizens as a means of capturing their votes, it is possible that the provision of maternal and neonatal health services increases prior to an election. Improved management may also take place around elections. One of the problems with public health services in many developing countries is absenteeism among medical staff (Chaudhury, Hammer, Kremer, Muralidharan, and Rogers, 2006). While no studies have addressed whether this is the case, absenteeism may very well decline during election years if politicians are able to exert a sufficient amount of pressure on lower-level bureaucrats to operate more effectively. Survey data from Chaudhury, Hammer, Kremer, Muralidharan, and Rogers (2006) suggest that 35 percent of health workers do not show up for work in health facilities across Bangladesh, India, Indonesia, Peru, and Uganda.

A fast-growing literature evaluates how conditional cash transfer programs and other government expenditures influence individual outcomes. Some of this literature evaluates election-year effects on expenditures. Schady (2000) finds that transfer payments through the Peruvian Social Fund (Fondo Nacional de Cooperación y Desarrollo Social, or FONCODES) increased prior to national elections. FONCONDES projects were targeted in provinces 
throughout the country that were most politically beneficial (because of supporters and marginal voters) as well as in those provinces that were the poorest. Community-based projects that were funded by FONCONDES include efforts to improve nutrition, family planning, access to credit, education, health, water and sanitation, school breakfasts, and other development objectives.

Kudamatsu (2011) provides suggestive evidence that democratization in Africa was associated with increases in breastfeeding and access to improved sanitation facilities. This may also be manifested in the run up to an election. The evidence put forth by Kudamatsu (2011) is consistent with the hypothesis that political incentives (particularly those for re-election in a newly formed democracy) will influence the provision of public or private goods that improve infant mortality rates in developing countries. However, this evidence also suggests that election cycles may have a very long lagged effect on infant mortality rates, as improvements in infrastructure like sanitation facilities or the diffusion of information about ways to prevent infant malnutrition or disease may take a long time to influence infant mortality rates.

Potrafke (2010) finds evidence of electoral cyclicality in public health expenditures among OECD countries. Because political budget cycles are larger in developing countries than in developed countries (Shi and Svensson, 2006), there may also be electoral cyclicality in public health expenditures across the developing world. However, the time series data on public health expenditures that are available for the developing world are not of a sufficient length to investigate election-year effects, thus this connection is somewhat speculative.

Cole (2009) finds that government-owned banks in India increase the provision of agricultural credit much more during election years than in years following elections. In examining the political budget cycle at the local level, Drazen and Eslava (2010) find that prior to municipal elections in Colombia expenditures on infrastructure (roads, power and water 
plants) increase significantly. Schuknecht (1996) argues that political budget cycles may be somewhat stronger in developing countries because of the lack of institutional development.

\subsubsection{Political Instability and Repression}

In some countries there has been violence before and/or after elections. If there is voter intimidation, repression of protests, or other forms of political instability and violence surrounding the holding of an election ${ }^{36}$, then infant mortality rates may actually increase before or after an election. Hafner-Burton, Hyde, and Jablonski (2010), for instance, argue that governments may use state-sponsored political repression during electoral cycles if there are few constraints on their behavior and there is a political opposition that poses a threat to their power. These forms of political instability may be manifested in higher infant mortality rates. ${ }^{37}$

Another reason to expect an electoral cycle in infant mortality rates (particularly after an election) is because of the political instability that often accompanies the end of electoral cycles in a number of developing countries. This was most recently evinced after the 2007 elections in Kenya, where hundreds of people were killed in the ensuing post-electoral violence. However, the experience of post-election political violence is not limited to Kenya and a number of developing countries have experienced violence right after (or even before) an election. Zimbabwe's 2000 parliamentary election, for instance, was marked by violence (Laakso, 2002).

Further, it may be the case that repression or voter intimidation drives increases in infant mortality in the run up to an election. Violence against opposition supporters or protestors may occur. Clamp-downs on free speech or free media may hinder the spread of information in a

\footnotetext{
${ }^{36}$ See Laakso (2002) in the context of parliamentary elections in Zimbabwe.

37 This illustrates the importance of considering the role played by electoral competitiveness, the level of democracy, and other political institutional aspects like whether the country has a presidential or parliamentary system of government or a majoritarian electoral rule. In many cases, 'sham' elections are held by unaccountable rulers who want to signal their openness or competitiveness, even though the actual elections are fraudulent and biased.
} 
society. There may also be post-electoral repression of protests against fraud or ballot tampering which harm individual citizens. Public health crises may increase and hospitals may become overwhelmed.

Then again, crime rates may fall prior to elections. Ghosh (2006) uses annual data from states in India to assess whether there are electoral cycles in crime rates across the country. The evidence suggests that property crimes and violent crimes both decline prior to elections.

These effects suggest that the degree of democracy or electoral competitiveness may matter for whether we observe election year effects in infant mortality rates. Yet countries that are relatively autocratic may hold elections as well, regardless of their competitiveness. There is evidence for electoral cycles under competitive authoritarian regimes in Mexico under the PRI (Grier and Grier, 2000; Gonzalez, 2002), Russia between 1995 and 2003 (Akhmedov and Zhuravskaya, 2004), Egypt under Hosni Mubarak (Blaydes, 2006), and Malaysia from 1967 to 1997 (Pepinsky, 2007).

Because the expected sign and magnitude of the estimated effect of electoral cycles on infant mortality are unknown a priori, the relationship between elections and infant mortality is ultimately an empirical question. The results of this investigation could indeed be counterintuitive. Grier and Grier (2000), for instance, find evidence of a postelection economic collapse in Mexico, but no expansion in economic activity prior to the election. Similarly, it could be the case that infant mortality does not decrease prior to an election (because of a long and variable lagged effect, for instance, or because there simply is no impact).

To test whether there is indeed electoral cyclicality in infant mortality rates, I employ a cross-country, micro-level panel dataset that covers more than 2.5 million infants across 70 developing countries between 1975 and 2009. I first consider what happens to infant mortality in 
a given country and year if there are elections held during that year. I also exploit the fact that the data on election dates and infant birth dates are both identifiable at the monthly level to explore heterogeneous effects of electoral cycles on either in utero or neonatal/post-neonatal exposure to electoral cycles. Further, because electioneering will vary across political contexts (Franzese, 2002), I consider the roles played by electoral competitiveness and the overall level of democracy.

\subsection{Data}

Micro-level data on infant mortality come from the Demographic and Health Surveys (DHS). ${ }^{38}$ The data are collected by assigning sampling clusters (for instance, towns or a collection of villages) in a country and then sampling households within these clusters. For the individuallevel recode files that I use in this paper, the surveys are directly administered to women ages 1549. The nationally-representative data allow for identification of birth dates for all live births (at the monthly level), the date of death (if applicable) and other infant, mother, and household specific characteristics through the use of a retrospective birth history component of the surveys. Because survey protocols are standardized across countries, these datasets can be combined to produce a cross-country panel on health outcomes. Using the DHS data, I therefore develop an unbalanced panel of more than 2.5 million infants across 70 developing countries. Information on the countries, survey years, and birth year coverage are presented in Table 3.1.

\footnotetext{
${ }^{38}$ http://www.measuredhs.com
} 
Table 3.1: Developing Countries in Sample

\begin{tabular}{|c|c|c|c|}
\hline Country & $\begin{array}{c}\text { Survey } \\
\text { Years }\end{array}$ & Birth Year Coverage & Obs \\
\hline Armenia & 2000,2005 & 1989-2004 & 7207 \\
\hline Azerbaijan & 2006 & $1995-2005$ & 4749 \\
\hline Bangladesh & $1994 ; 1996-97 ; 1999-2000 ; 2004 ; 2007$ & $1982-2006$ & 69504 \\
\hline Benin & $1996 ; 2001 ; 2006$ & $1985-2005$ & 48539 \\
\hline Bolivia & 1989; 1994; 1998; 2003; 2008 & $1978-2007$ & 75157 \\
\hline Brazil & $1986 ; 1991 ; 1996$ & 1975-1995 & 24307 \\
\hline Burkina Faso & $1993 ; 1999 ; 2003$ & $1981-2002$ & 41650 \\
\hline Burundi & 1987 & 1976-1986 & 6507 \\
\hline Cambodia & $2000 ; 2005$ & $1989-2005$ & 36629 \\
\hline Cameroon & $1991 ; 1998 ; 2004$ & $1980-2003$ & 28074 \\
\hline Cent. Af. Rep. & 1994-95 & 1983-1994 & 8831 \\
\hline Chad & $1996-97 ; 2004$ & $1985-2003$ & 24220 \\
\hline Colombia & $1986 ; 1990 ; 1995 ; 2000 ; 2005$ & $1975-2009$ & 97228 \\
\hline Comoros & 1996 & 1985-1995 & 3830 \\
\hline Congo & 2005 & 1994-2004 & 8199 \\
\hline Congo (DRC) & 2007 & 1996-2006 & 15164 \\
\hline Cote d'Ivoire & $1994 ; 1998-99$ & $1983-1998$ & 16842 \\
\hline Dom. Rep. & 1986; 1991; 1996; 1999; 2002; 2007 & $1975-2006$ & 72949 \\
\hline Ecuador & 1987 & $1975-1986$ & 5837 \\
\hline Egypt & $1988 ; 1992 ; 1995 ; 2000 ; 2003 ; 2005 ; 2008$ & $1977-2007$ & 140507 \\
\hline El Salvador & 1985 & $1975-1984$ & 4747 \\
\hline Ethiopia & 2000 & 1989-1999 & 20557 \\
\hline Gabon & 2001 & 1989-1999 & 8081 \\
\hline Ghana & $1993 ; 1998 ; 2003 ; 2008$ & $1977-2007$ & 32387 \\
\hline Guatemala & $1987 ; 1995 ; 1998-99$ & 1976-1998 & 35560 \\
\hline Guinea & $1999 ; 2005$ & $1988-2004$ & 24423 \\
\hline Haiti & $1994-95 ; 2000 ; 2005-06$ & $1983-2005$ & 29926 \\
\hline Honduras & $2005-06$ & $1994-2005$ & 22484 \\
\hline India & $1993 ; 1998-99 ; 2005-2006$ & $1981-2005$ & 359020 \\
\hline Indonesia & $1987 ; 1991 ; 1994 ; 1997 ; 2002-03 ; 2007$ & 1976-2006 & 191944 \\
\hline Jordan & $1990 ; 1997 ; 2002 ; 2007$ & 1979-2008 & 76156 \\
\hline Kazakhstan & $1995 ; 1999$ & 1984-1998 & 6534 \\
\hline Kenya & $1989 ; 1993 ; 1998 ; 2003 ; 2008-09$ & $1977-2008$ & 57071 \\
\hline Kyrgyz Rep. & 1997 & $1986-1996$ & 4074 \\
\hline Lesotho & $2004 ; 2009$ & $1993-2008$ & 12993 \\
\hline Liberia & $1986 ; 2007$ & $1975-2006$ & 19329 \\
\hline Madagascar & $1992 ; 1997 ; 2003-04 ; 2008-09$ & $1981-2008$ & 54318 \\
\hline Malawi & 2004 & $1981-2004$ & 46453 \\
\hline Mali & $1995-96 ; 2001 ; 2006$ & $1976-2005$ & 76827 \\
\hline Mexico & 1987 & $1976-1986$ & 10213 \\
\hline Moldova & 2005 & $1994-2004$ & 3124 \\
\hline Morocco & $1987 ; 1992 ; 2003-04$ & $1976-2003$ & 34159 \\
\hline Mozambique & $1997 ; 2003$ & $1986-2002$ & 31027 \\
\hline Namibia & $1992 ; 2000 ; 2006-07$ & $1981-2006$ & 23043 \\
\hline Nicaragua & $1998 ; 2001$ & $1986-2000$ & 31480 \\
\hline Niger & $1992 ; 1998 ; 2006$ & $1981-2005$ & 45284 \\
\hline
\end{tabular}




\begin{tabular}{lccc} 
Nigeria & $1990 ; 1999 ; 2003 ; 2008$ & $1979-2007$ & 89498 \\
Pakistan & $1990-91 ; 2006-07$ & $1979-19901995-2006$ & 32774 \\
Paraguay & 1990 & $1979-1989$ & 7778 \\
Peru & $1986 ; 1991-92 ; 1996 ; 2000 ; 2007-08$ & $1975-2007$ & 120826 \\
Philippines & $1993 ; 1998 ; 2003 ; 2008$ & $1982-2007$ & 59111 \\
Rwanda & $1992 ; 2000 ; 2005 ; 2007-08$ & $1981-2007$ & 47950 \\
Senegal & $1986 ; 1992-93 ; 1997 ; 2005$ & $1975-2004$ & 51425 \\
Sierra Leone & 2008 & $1997-2007$ & 10897 \\
South Africa & 1998 & $1986-1997$ & 10000 \\
Sri Lanka & 1987 & $1975-1986$ & 8244 \\
Sudan & $1989-1990$ & $1978-1989$ & 12664 \\
Swaziland & $2006-07$ & $1995-2006$ & 5065 \\
Tanzania & 1987 & $1980-2004$ & 46639 \\
Thailand & 1998 & $1976-1986$ & 7531 \\
Togo & 1987 & $1977-1997$ & 18700 \\
Trinidad & 1988 & $1976-1986$ & 3636 \\
Tunisia & $1991-92 ; 1996 ; 1999 ; 2004-05$ & $1977-1987$ & 8282 \\
Turkey & $1993 ; 1998 ; 2003$ & $1982-2003$ & 24526 \\
Uganda & 2007 & $1977-2005$ & 48144 \\
Ukraine & 1996 & $1996-2006$ & 2580 \\
Uzbekistan & $1997 ; 2002$ & $1985-1995$ & 4744 \\
Vietnam & $1991-1992$ & $1986-2001$ & 12688 \\
Yemen & $1988-89 ; 1995 ; 2000-01 ; 2006$ & 1990 & 1072 \\
Zambia & $1992 ; 1996 ; 2001-02 ; 2007$ & $1980-2006$ & 46082 \\
Zimbabwe & $1989 ; 1994 ; 1999 ; 2005-06$ & $1977-2005$ & 29842 \\
\hline
\end{tabular}

From the DHS data, I construct a binary indicator, mortality, which takes on a value of ' 1 ' if child $i$ dies before or at the age of one year and a value of ' 0 ' if otherwise ${ }^{39}$ Because I am interested in how electoral cycles influence infant mortality rates in terms of deaths per 1,000 live births, I multiply this measure by 1,000 . The coefficient estimates below are therefore in terms of infant deaths per 1,000 live births.

Information on election dates come from the Database of Political Institutions. I construct a binary indicator, election, which takes on a value of ' 1 ' if there is an election in a particular year in a given country and ' 0 ' otherwise. ${ }^{40} \mathrm{I}$ interact this variable with a number of measures of political institutions to examine whether different types of political institutions matter for the

\footnotetext{
${ }^{39}$ Because of potential 'age heaping' of the infant mortality data at 12 months (i.e., because the DHS data are retrospective birth histories, mothers may 'round up' the dates of the deaths of their children), I define infant mortality as death within the first 12 months of life.

${ }^{40}$ Using this definition, about 25 percent of all year observations are election years.
} 
effects of elections on infant mortality rates (e.g., presidential versus parliamentary systems of government).

I also use a measure of electoral competitiveness from the Database of Political Institutions, competitiveness, which measures the degree of electoral competitiveness and ranges from 0 (completely non-competitive) to 7 (competitive) ${ }^{41}$ Electoral cyclicality in infant mortality rates may only be likely if elections are sufficiently competitive, thus it is important to consider the interaction between elections and electoral competitiveness. I also obtain information on political institutions from the Polity IV database. I use the polity2 regime measure, which takes on a value of -10 for completely autocratic regimes and a value of +10 for completely democratic regimes. Across the entire panel, the average polity2 score is 1.13 .

Data on real GDP per person and growth in GDP per person come from the World Bank (2011). The level of income per person is in terms of U.S. dollars (2000) and is adjusted for differences in purchasing power across countries (PPP). The logarithm of real GDP per person is used in all specifications below. I use a number of control variables in the analysis below. In addition to the level and growth rate of real GDP per person, I control for whether the household is located in a rural area of the country, an indicator for male gender, an indicator for multiple births, and cubic polynomials in mother's age at the time of birth, years of education, and infant birth order (Baird, Friedman, and Schady, 2011). All regressions include a constant term which is not reported in the tables below.

Summary statistics are presented in Table 3.2. Birth years span from 1975 to 2009 across the sample, with the bulk of birth years in the panel taking place in the mid to late 1980s and

\footnotetext{
${ }^{41}$ Specifically, I use the competitiveness score for executive elections if only executive elections took place and the competitiveness score for legislative elections if only legislative elections took place. If both executive and legislative elections took place at the same time (as is the case for parliamentary systems of government), I take the row mean of observations of the competitiveness scores for executive and legislative elections.
} 
throughout the 1990s. Across the entire panel, the average infant mortality rate is 78 deaths per 1,000 live births. Splitting along election years, the average rate is about 74.6 per 1,000 during election years and the average rate is 78.9 per 1,000 during non-election years. Among countryyear observations where the Polity score is strictly greater than zero (i.e., relatively more democratic country-year observations), the average rate is about 68.8 per 1,000 during election years and 71.3 during non-election years. When the Polity score is less than or equal to zero (i.e. relatively more autocratic), the average annual infant mortality rate is 84 per 1,000 during election years and 87.1 during non-election years. These summary statistics suggest that infant mortality rates are relatively lower during election years even across different levels of democracy. Countries that are more autocratic have much higher infant mortality rates (see Kudamatsu, 2011 for a discussion). Across the entire panel, electoral competitiveness is surprisingly low-on average, most countries are closer to having low levels of electoral competitiveness (1.4 on a scale of 0 to 7 ). Most country-year observations considered in the sample are for presidential systems of government (about 64 percent) while the other observations are close to evenly split between parliamentary systems of government (about 20 percent) and assembly-elected presidential systems of government (about 16 percent). Most individuals live in relatively more rural areas of the country. Average age at birth is about 26 years, with a standard deviation of around 6 years. Most mothers in the sample have relatively few years of education (about 4.3) and the standard deviation is larger than the mean (4.5). 
Table 3.2: Summary Statistics

\begin{tabular}{|c|c|c|c|c|c|}
\hline & \# Obs. & Mean & Std. Dev. & Min & Max \\
\hline Infant Mortality & 2707842 & .0780777 & .2682938 & 0 & 1 \\
\hline Infant Mortality (x 1000) & 2707842 & 78.07767 & 268.2938 & 0 & 1000 \\
\hline Election Year & 2668705 & .2478592 & .4317697 & 0 & 1 \\
\hline Months In Utero Exposure & 2702777 & 2.147836 & 3.160408 & 0 & 9 \\
\hline Election Competitiveness & 2691070 & 1.422577 & 2.649814 & 0 & 7 \\
\hline Parliamentary System & 2668705 & .1995968 & .3996974 & 0 & 1 \\
\hline Presidential System & 2668705 & .6381421 & .4805381 & 0 & 1 \\
\hline Assembly-Elected President & 2668705 & .1622611 & .3686902 & 0 & 1 \\
\hline Polity & 2693519 & 1.129213 & 6.4115 & -10 & 9 \\
\hline Rural & 2702689 & .6424524 & .479278 & 0 & 1 \\
\hline Male & 2707842 & .510922 & .4998808 & 0 & 1 \\
\hline Real GDP per person, PPP & 2572554 & 2347.76 & 2012.033 & 150.807 & 16015.66 \\
\hline Log real GDP per person, PPP & 2572554 & 7.458703 & .7700905 & 5.016001 & 9.681322 \\
\hline GDP per person, growth rate & 2657401 & 1.635362 & 5.104959 & -46.89249 & 90.13997 \\
\hline Age at first birth & 2707842 & 25.61059 & 5.874245 & 9.083333 & 39 \\
\hline Birth order & 2707842 & 3.283599 & 2.236269 & 1 & 27 \\
\hline Years of education & 2705714 & 4.297786 & 4.541368 & 0 & 27 \\
\hline Multiple birth & 2707842 & .0243825 & .1542336 & 0 & 1 \\
\hline Number of births & 2522130 & 2.899063 & 1.466359 & 1 & 16 \\
\hline $\begin{array}{l}\text { Infant Mortality during Election } \\
\text { Years (x 1000) }\end{array}$ & 661463 & 74.56955 & 262.6957 & & \\
\hline $\begin{array}{l}\text { Infant Mortality during Non-election } \\
\text { Years (x 1000) }\end{array}$ & 2007242 & 78.86393 & 269.5263 & & \\
\hline $\begin{array}{l}\text { Infant Mortality during Election } \\
\text { Years; Polity > } 0 \text { (x 1000) }\end{array}$ & 409751 & 68.76371 & 253.0522 & & \\
\hline $\begin{array}{l}\text { Infant Mortality during Non-election } \\
\text { Years; Polity }>0(x \text { 1000) }\end{array}$ & 1047481 & 71.34831 & 257.4059 & & \\
\hline $\begin{array}{l}\text { Infant Mortality during Election } \\
\text { Years; Polity }<=0 \text { (x 1000) }\end{array}$ & 251712 & 84.02063 & 277.4193 & & \\
\hline $\begin{array}{l}\text { Infant Mortality during Non-election } \\
\text { Years; Polity }<=0(x \text { 1000) }\end{array}$ & 959761 & 87.06647 & 281.9326 & & \\
\hline
\end{tabular}

While the DHS data provide a rich source of information on individual health outcomes across the developing world, they do have their limitations. The DHS data are retrospective in nature, which suggests that there may be a few issues with measurement. For instance, there may 
be 'recall bias' where a mother cannot recall the precise month in which her child was born. This is likely to be problematic for births that occurred a decade or longer ago. Following Baird, Friedman and Schady (2011), I only consider infants who are born within 11 years of survey administration. I also discard births to women age 40 or older to avoid issues regarding the varying age composition of mothers. I do not use information in infants who were born within less than one year of survey administration because of issues with left censoring of the data.

Another potential limitation of using the DHS data is that infant mortality data are not available for infants or children of women who had died, hence the potential for mortality-related selection bias. For instance, it is estimated that millions have died in the Democratic Republic of the Congo (DRC) over the past decade, mainly due to public health crises, malnutrition, and poor living standards stemming from the civil wars and political instability. It is likely that the infant mortality statistics for the DRC and other countries experiencing severe civil war are biased downward (in the sense that infant mortality rates may appear to be lower than they actually are) due to mortality-related selection bias.

\subsection{Methods}

To estimate the impact of elections on the likelihood of mortality of infant $i$ born to household/mother $h$ in country $c$ in birth year $y$, I use the following linear probability model: ${ }^{42}$

$$
\text { mortality }_{\text {ihcy }}=\alpha+\text { Belection }_{\text {cy }}+\mathrm{Z}_{\text {ih }}^{\prime} \gamma+\mathrm{X}_{\mathrm{cy}}^{\prime} \delta+\varepsilon_{\text {ihcy }}
$$

where election ${ }_{\text {cy }}$ takes on a value of ' 1 ' if there is an election in country $c$ in year $y$ and a value of ' 0 ' otherwise, $Z_{\mathrm{ih}}$ is a vector of individual- and household-level characteristics, $\mathrm{X}_{\mathrm{cy}}$ is a vector of country and year-specific variables, and $\varepsilon_{\text {ihcy }}$ is the error term. The vector of individual and

\footnotetext{
${ }^{42}$ The use of a linear probability model in this context follows the work of Bhalotra (2010), Kudamatsu (2011), Kudamatsu, Perrson, and Stromberg (2011).
} 
household characteristics, $\mathrm{Z}_{\mathrm{ih}}$, includes whether the household is located in a rural area of the country at the time of interview, the gender of the infant, whether the infant was a multiple birth (i.e. twin, triplet, etc.), and cubic polynomials in mother's age at the time of birth, years of education, and infant birth order. ${ }^{43}$

The vector of country and year-specific variables, $\mathrm{X}_{\mathrm{cy}}^{\prime}$, includes the log of real per capita income (constant, 2000 US\$, adjusted for purchasing power differences across countries) and the growth rate of GDP per capita. Because elections are not randomly assigned and the election year effects may pick up the impact of democracy on infant mortality rates, I also include the Polity score of autocracy/democracy in the vector of country and year-specific variables. ${ }^{44}$ Observations of the error term are clustered at the country level to control for unobserved similarities among infants born within the same country as well as to ensure that the estimates are robust to heteroskedasticity and serial correlation. The coefficient of interest is $\beta$, which measures the impact of having an election in country $c$ in year $y$ on infant mortality conditional on the included covariates.

To control for unobserved heterogeneity across countries, I estimate the linear probability model above with country fixed effects:

$$
\text { mortality }_{\text {ihcy }}=\eta_{\mathrm{c}}+\text { Belection }_{\mathrm{cy}}+\mathrm{Z}_{\mathrm{ih}}^{\prime} \gamma+\mathrm{X}_{\mathrm{cy}}^{\prime} \delta+\varepsilon_{\mathrm{ihcy}}
$$

By including country-specific fixed effects, I am able to identify the effect of an election on infant mortality from the deviation within each country from the mean infant mortality rate. Because of how the DHS data are structured, I am also able to include mother-level fixed effects. While I control for mother's education, birth order, gender, location of the home (i.e., rural

\footnotetext{
${ }^{43}$ See Baird, Friedman, and Schady (2011).

${ }^{44}$ Specifically, I use the polity 2 measure.
} 
versus urban), and other observable characteristics, allowing the panel unit to be the mother takes all unobserved heterogeneity at the household or mother level into account:

$$
\text { mortality }_{\text {ihcy }}=\mathrm{l}_{h}+\theta_{\mathrm{y}}+\text { Belection }_{\mathrm{cy}}+\mathrm{Z}_{\mathrm{ih}}^{\prime} \gamma+\mathrm{X}_{\mathrm{cy}}^{\prime} \delta+\varepsilon_{\text {ihcy }}
$$

Here, I identify the effect of an election on infant mortality by the deviation within each household/mother from the household mean. The inclusion of mother fixed effects helps control for endogenous selection into the full sample of births (Bhalotra, 2010; Baird, Friedman, and Schadu, 2011). While the mother fixed effects panel regression approach allows for the control of unobserved, time-invariant mother characteristics, it does have the disadvantage of limiting the sample to those women who have had more than one live birth. I present pooled OLS regression results from the restricted sample in order to compare the results between the restricted sample and the full sample. This is an unbalanced panel in that the unit of observation is the mother/household and the time dimension is the birth year for every child. I also include either birth year fixed effects or country-specific linear trends in the specifications reported in Tables 3-6 below.

In a number of specifications, I interact the election variable with the electoral competitiveness variable to allow the impact of elections on infant mortality to differ depending on how competitive each election is. Including this interaction effect is also important to prevent confounding the effects of institutional quality or competitiveness with elections (see, for instance, Persson and Tabellini, 2005). When elections are not free and fair, there may be violent election campaigning (as in the case of Zimbabwe in the 2000 parliamentary election [Laakso, 2002]) or few incentives for incumbent politicians or rulers to provide benefits to citizens. However, a literature examines some of the other explanations for increases in transfer payments and government spending prior to elections even in authoritarian regimes where there is little 
electoral competition. The reasons for these fiscal policies may be oriented around a regime seeking legitimacy or control over an opposition group, even if the elections are not per se competitive.

\subsection{Results}

The baseline results are presented in Table $3.3 .^{45}$ When only considering the pooled OLS regression results, it appears that the holding of any election is associated with a reduction in infant mortality of about 2.4 per 1,000 (Table 3.3, Panel A). However, the coefficient from the panel regression results using country fixed effects is about half this magnitude $(1.3$ per 1,000$)$ and the impact is generally minor in magnitude and statistically insignificant when including birth year fixed effects, time trends, or mother fixed effects. When birth year fixed effects are included in the regressions, I find no evidence that infant mortality rates change over the electoral cycle. However, I also find no evidence that the overall level of income matters for infant mortality when the birth year fixed effects are included, which suggests that the secular decline in infant mortality rates due to technology and information diffusion may matter more than the overall level of income for explaining infant mortality rates. When I include the Polity score as a covariate, the magnitude of the electoral year effects is diminished. The statistical significance and magnitude of democracy is seriously mitigated when year fixed effects or country-specific linear time trends are considered as additional covariates.

\footnotetext{
${ }^{45}$ In all tables, the results are presented as follows: column 1 presents estimates from pooled OLS regressions; column 2 presents estimates from country fixed effects panel regressions; column 3 includes birth year fixed effects along with country fixed effects; column 4 allows for country specific linear trends in infant mortality in addition to the country fixed effects; column 5 presents results from pooled OLS regressions for which the sample is restricted to only those mothers who have had at least two births; column 6 presents results from mother fixed effects panel regressions, column 7 augments these regressions with birth year fixed effects, and lastly, column 8 allows for the inclusion of country-specific linear trends along with mother fixed effects. Panel A presents the results without controlling for the Polity score while Panel B presents the results when controlling for Polity.
} 


\section{Table 3.3: Election Year Effects on Infant Mortality Rates}

Panel A: Election Year Effects

\begin{tabular}{lcccccccc}
\hline & $(1)$ & $(2)$ & $(3)$ & $(4)$ & $(5)$ & $(6)$ & $(7)$ & $(8)$ \\
\hline Election & $-2.405^{* * *}$ & $-1.293^{* *}$ & -0.066 & -0.209 & $-2.539^{* *}$ & -0.646 & -0.579 & -0.639 \\
& $(0.911)$ & $(0.538)$ & $(0.501)$ & $(0.610)$ & $(0.978)$ & $(0.731)$ & $(0.745)$ & $(0.740)$ \\
\hline Covariates & YES & YES & YES & YES & YES & YES & YES & YES \\
Country FE & NO & YES & YES & YES & NO & NO & NO & NO \\
Mother FE & NO & NO & NO & NO & NO & YES & YES & YES \\
Birth-year FE & NO & NO & YES & NO & NO & NO & YES & NO \\
Trends & NO & NO & NO & YES & NO & NO & NO & YES \\
Sample & Full & Full & Full & Full & Restricted & Restricted & Restricted & Restricted \\
\# Countries & 69 & 69 & 69 & 69 & 69 & 69 & 69 & 69 \\
\# Observations & 2522130 & 2522130 & 2522130 & 2522130 & 2088328 & 2088328 & 2088328 & 2088328 \\
\hline
\end{tabular}

Panel B: Controlling for Polity Score

\begin{tabular}{lcccccccc}
\hline & $(1)$ & $(2)$ & $(3)$ & $(4)$ & $(5)$ & $(6)$ & $(7)$ & $(8)$ \\
\hline Election & $-1.501^{*}$ & -0.391 & -0.064 & -0.282 & $-1.604^{*}$ & -0.376 & -0.377 & -0.600 \\
& $(0.845)$ & $(0.460)$ & $(0.516)$ & $(0.616)$ & $(0.916)$ & $(0.740)$ & $(0.748)$ & $(0.745)$ \\
& & & & & & & \\
Level of & $-0.633^{* *}$ & $-1.036^{* * *}$ & -0.003 & 0.116 & $-0.661^{* *}$ & $-0.472^{* * *}$ & $-0.335^{*}$ & -0.063 \\
Democracy & $(0.270)$ & $(0.310)$ & $(0.260)$ & $(0.165)$ & $(0.283)$ & $(0.169)$ & $(0.169)$ & $(0.133)$ \\
& & & & & & & & \\
\hline Covariates & YES & YES & YES & YES & YES & YES & YES & YES \\
Country FE & NO & YES & YES & YES & NO & NO & NO & NO \\
Mother FE & NO & NO & NO & NO & NO & YES & YES & YES \\
Birth-year FE & NO & NO & YES & NO & NO & NO & YES & NO \\
Trends & NO & NO & NO & YES & NO & NO & NO & YES \\
Sample & Full & Full & Full & Full & Restricted & Restricted & Restricted & Restricted \\
\# Countries & 69 & 69 & 69 & 69 & 69 & 69 & 69 & 69 \\
\# Observations & 2522130 & 2522130 & 2522130 & 2522130 & 2088328 & 2088328 & 2088328 & 2088328 \\
\hline
\end{tabular}

Notes: The dependent variable is a binary indicator of infant mortality (multiplied by 1,000). Standard errors clustered at the country level are reported in parentheses. Statistical significance is indicated as follows: $* \mathrm{p}<0.10$, $* * \mathrm{p}<0.05, * * * \mathrm{p}<0.01$. Covariates include the level and growth rate of GDP per person (log terms, constant 2000 U.S. dollars, adjusted for purchasing power differences across countries), whether the household is located in a rural area of the country, an indicator for male gender, an indicator for multiple births, and cubic polynomials in mother's age at the time of birth, years of education, and infant birth order. A constant term is included in each regression but is not reported.

To control for how different political institutions may influence whether there are electoral cycles in infant mortality, I interact the election variable with a measure of electoral competitiveness from the Database of Political Institutions. Recall that this measure captures the degree of competitiveness of an election, ranging from 0 (completely non-competitive) to 7 
(competitive). Table 3.4 presents the results form regressions with the elections and the interaction effects. I present the F-test statistics which indicate the joint statistical significance between the election variable and the interaction variable. Generally, the results are only jointly statistically significant when considering the pooled OLS regressions for the full and restricted samples and the country fixed effects panel regressions. The results indicate that what may matter for whether there are election year influences on infant mortality rates is whether there is a high degree of electoral competition. Without competition, there are weaker incentives for incumbent politicians to pursue expansionary policies or policies aimed at providing the rural poor with public and private goods and services in order to 'buy' their votes. 


\section{Table 3.4: Election Year Effects on Infant Mortality Rates}

\section{Panel A: Election Year Effects and Interaction with Electoral Competitiveness}
(1)
(2)
(3)
(4)
(5)
(6)
(7)
(8)

\begin{tabular}{|c|c|c|c|c|c|c|c|c|}
\hline Election & $\begin{array}{c}16.714^{* * * * *} \\
(4.515)\end{array}$ & $\begin{array}{l}9.734^{* * * *} \\
(3.245)\end{array}$ & $\begin{array}{c}4.560 \\
(3.516)\end{array}$ & $\begin{array}{c}1.923 \\
(3.009)\end{array}$ & $\begin{array}{c}16.819^{* * *} \\
(4.900)\end{array}$ & $\begin{array}{c}2.467 \\
(2.319)\end{array}$ & $\begin{array}{c}1.497 \\
(2.346)\end{array}$ & $\begin{array}{c}2.227 \\
(2.220)\end{array}$ \\
\hline Elect x Comp & $\begin{array}{c}-3.262^{* * *} \\
(0.778)\end{array}$ & $\begin{array}{c}-1.888^{* * *} \\
(0.577)\end{array}$ & $\begin{array}{c}-0.799 \\
(0.609)\end{array}$ & $\begin{array}{c}-0.366 \\
(0.524)\end{array}$ & $\begin{array}{c}-3.333^{* * * *} \\
(0.846)\end{array}$ & $\begin{array}{c}-0.542 \\
(0.394)\end{array}$ & $\begin{array}{c}-0.364 \\
(0.400)\end{array}$ & $\begin{array}{c}-0.498 \\
(0.386)\end{array}$ \\
\hline F-Test & $10.02^{* * * *}$ & $5.74^{* * * *}$ & 0.86 & 0.27 & $9.06^{* * *}$ & 1.23 & 0.66 & 1.05 \\
\hline Covariates & YES & YES & YES & YES & YES & YES & YES & YES \\
\hline Country FE & NO & YES & YES & YES & NO & NO & NO & NO \\
\hline Mother FE & $\mathrm{NO}$ & NO & NO & NO & NO & YES & YES & YES \\
\hline Birth-year FE & $\mathrm{NO}$ & $\mathrm{NO}$ & YES & NO & NO & NO & YES & NO \\
\hline Trends & NO & $\mathrm{NO}$ & NO & YES & NO & NO & NO & YES \\
\hline Sample & Full & Full & Full & Full & Restricted & Restricted & Restricted & Restricted \\
\hline \# Countries & 69 & 69 & 69 & 69 & 69 & 69 & 69 & 69 \\
\hline \# Observations & 2522130 & 2522130 & 2522130 & 2522130 & 2088328 & 2088328 & 2088328 & 2088328 \\
\hline
\end{tabular}

Panel B: Controlling for Polity Score

\begin{tabular}{|c|c|c|c|c|c|c|c|c|}
\hline & (1) & (2) & (3) & (4) & (5) & (6) & (7) & (8) \\
\hline Election & $\begin{array}{c}12.991^{* * * * *} \\
(3.702)\end{array}$ & $\begin{array}{l}8.930^{* * * * *} \\
(3.291)\end{array}$ & $\begin{array}{c}4.562 \\
(3.524)\end{array}$ & $\begin{array}{c}1.765 \\
(2.969)\end{array}$ & $\begin{array}{c}12.945^{* * * * *} \\
(4.095)\end{array}$ & $\begin{array}{c}2.972 \\
(2.313)\end{array}$ & $\begin{array}{c}1.901 \\
(2.337)\end{array}$ & $\begin{array}{c}2.341 \\
(2.186)\end{array}$ \\
\hline Elect x Comp & $\begin{array}{c}-2.488^{* * *} \\
(0.665)\end{array}$ & $\begin{array}{c}-1.600^{* * *} \\
(0.577)\end{array}$ & $\begin{array}{l}-0.799 \\
(0.609)\end{array}$ & $\begin{array}{c}-0.351 \\
(0.520)\end{array}$ & $\begin{array}{c}-2.521^{* * *} \\
(0.742)\end{array}$ & $\begin{array}{l}-0.583 \\
(0.397)\end{array}$ & $\begin{array}{l}-0.399 \\
(0.400)\end{array}$ & $\begin{array}{l}-0.511 \\
(0.382)\end{array}$ \\
\hline $\begin{array}{l}\text { Level of } \\
\text { Democracy }\end{array}$ & $\begin{array}{c}-0.570^{* * *} \\
(0.263) \\
\end{array}$ & $\begin{array}{c}-1.012^{* * * *} \\
(0.308)\end{array}$ & $\begin{array}{l}-0.003 \\
(0.259) \\
\end{array}$ & $\begin{array}{c}0.111 \\
(0.163) \\
\end{array}$ & $\begin{array}{c}-0.595^{* *} \\
(0.279) \\
\end{array}$ & $\begin{array}{c}-0.476^{* * *} \\
(0.169) \\
\end{array}$ & $\begin{array}{c}-0.339^{* *} \\
(0.169) \\
\end{array}$ & $\begin{array}{l}-0.073 \\
(0.131) \\
\end{array}$ \\
\hline F-Test & $7.05^{* * *}$ & $3.85^{* * *}$ & 0.86 & 0.28 & $5.87^{* * * *}$ & 1.13 & 0.58 & 1.05 \\
\hline Covariates & YES & YES & YES & YES & YES & YES & YES & YES \\
\hline Country FE & NO & YES & YES & YES & NO & NO & NO & NO \\
\hline Mother FE & NO & NO & NO & NO & NO & YES & YES & YES \\
\hline Birth-year FE & NO & NO & YES & NO & NO & NO & YES & NO \\
\hline Trends & NO & NO & NO & YES & NO & NO & NO & YES \\
\hline Sample & Full & Full & Full & Full & Restrict & Restrict & Restrict & Restrict \\
\hline \# Countries & 69 & 69 & 69 & 69 & 69 & 69 & 69 & 69 \\
\hline \# Observations & 2522130 & 2522130 & 2522130 & 2522130 & 2088328 & 2088328 & 2088328 & 2088328 \\
\hline \multicolumn{9}{|c|}{$\begin{array}{l}\text { Notes: The dependent variable is a binary indicator of infant mortality (multiplied by } 1,000 \text { ). Standard errors } \\
\text { clustered at the country level are reported in parentheses. Statistical significance is indicated as follows: } * \mathrm{p}<0.10 \text {, } \\
* * \mathrm{p}<0.05, * * * \mathrm{p}<0.01 \text {. Covariates include the level and growth rate of GDP per person (log terms, constant } 2000 \\
\text { U.S. dollars, adjusted for purchasing power differences across countries), whether the household is located in a rural } \\
\text { area of the country, an indicator for male gender, an indicator for multiple births, and cubic polynomials in mother's } \\
\text { age at the time of birth, years of education, and infant birth order. A constant term is included in each regression but } \\
\text { is not reported. The F-test statistic is for the null hypothesis that the parameters on the election variable and the } \\
\text { interaction variable are both equal to zero. }\end{array}$} \\
\hline
\end{tabular}


Table 3.5 restricts the regressions for only those country-year observations with a Polity score greater than 0 . Incumbent politicians may be less prone to pursuing expansionary policies in the run up to an election if government institutions are relatively more autocratic. The coefficient estimates are much larger in magnitude, suggesting that countries with relatively more democratic institutions will experience stronger election year effects on infant mortality. This is an intuitive result considering that many purely autocratic countries put forth 'sham' elections as a means to signal competitiveness of executive recruitment and accountability to citizens. Column 7 presents evidence for mother fixed effects panel regressions with birth year fixed effects. The results suggest that the only time an electoral cycle would be associated with reductions in infant mortality is when the degree of electoral competitiveness is sufficiently high. However, the F-test statistic for joint significance is relatively low, so it is difficult to calculate with any great deal of precision how these effects are influencing infant mortality rates. The results from the country fixed effects panel regression estimates suggest that elections would have to be almost completely competitive for electoral cycles to exert any negative influence on infant mortality rates among those country-year observations where the Polity measure is strictly greater than zero. Additionally, once controlling for the impact of political regime (via Polity), there is less evidence of election year effects on infant mortality. 


\section{Table 3.5: Election Year Effects on Infant Mortality Rates - Interaction with Electoral Competitiveness Variable}

Panel A: Only for Country-Year Observations with Polity > 0

\begin{tabular}{lcccccccc}
\hline & $(1)$ & $(2)$ & $(3)$ & $(4)$ & $(5)$ & $(6)$ & $(7)$ & $(8)$ \\
\hline Election & $37.726^{* * *}$ & $15.134^{* * *}$ & 7.669 & -0.841 & $55.012^{* * *}$ & $11.878^{* * *}$ & $10.260^{* *}$ & 4.388 \\
& $(6.706)$ & $(4.728)$ & $(6.072)$ & $(8.157)$ & $(8.538)$ & $(5.560)$ & $(5.190)$ & $(4.776)$ \\
& & & & & & & & \\
Election X & $-6.160^{* * *}$ & $-2.411^{* * *}$ & -1.189 & 0.100 & $-8.786^{* * *}$ & $-1.714^{*}$ & $-1.414^{*}$ & -0.625 \\
Comp & $(1.033)$ & $(0.746)$ & $(1.019)$ & $(1.298)$ & $(1.246)$ & $(0.867)$ & $(0.820)$ & $(0.770)$ \\
& & & & & & & & \\
\hline F-Test & $18.04^{* * *}$ & $5.22^{* * *}$ & 0.99 & 0.06 & $26.31^{* * *}$ & $2.59^{*}$ & $2.50^{* *}$ & 0.57 \\
\hline Covariates & YES & YES & YES & YES & YES & YES & YES & YES \\
Country FE & NO & YES & YES & YES & NO & NO & NO & NO \\
Mother FE & NO & NO & NO & NO & NO & YES & YES & YES \\
Birth-year FE & NO & NO & YES & NO & NO & NO & YES & NO \\
Trends & NO & NO & NO & YES & NO & NO & NO & YES \\
Sample & Polity $>0$ & Polity $>0$ & Polity $>0$ & Polity $>0$ & Restricted & Restricted & Restricted & Restricted \\
& & & & & - Polity $>0$ & - Polity $>0$ & - Polity > 0 & - Polity $>0$ \\
\# Countries & 48 & 48 & 48 & 48 & 48 & 48 & 48 & 48 \\
\# Observations & 1421049 & 1421049 & 1421049 & 1421049 & 1090431 & 1090431 & 1090431 & 1090431 \\
\hline
\end{tabular}

Panel B: Controlling for Polity Score

\begin{tabular}{|c|c|c|c|c|c|c|c|c|}
\hline & (1) & (2) & (3) & (4) & (5) & (6) & (7) & (8) \\
\hline Election & $\begin{array}{c}35.695^{* * * * *} \\
(6.785)\end{array}$ & $\begin{array}{c}14.371^{* * * * *} \\
(4.748)\end{array}$ & $\begin{array}{c}7.422 \\
(6.119)\end{array}$ & $\begin{array}{l}-0.821 \\
(8.163)\end{array}$ & $\begin{array}{c}5^{52.185^{* * * *}} \\
(9.233)\end{array}$ & $\begin{array}{l}11.835^{* *} \\
(5.514)\end{array}$ & $\begin{array}{l}10.086^{*} \\
(5.179)\end{array}$ & $\begin{array}{c}4.394 \\
(4.795)\end{array}$ \\
\hline Election X Comp & $\begin{array}{c}-5.764^{* * * *} \\
(1.060)\end{array}$ & $\begin{array}{c}-2.325^{* * *} \\
(0.753)\end{array}$ & $\begin{array}{l}-1.170 \\
(1.023)\end{array}$ & $\begin{array}{c}0.087 \\
(1.298)\end{array}$ & $\begin{array}{c}-8.263^{* * *} \\
(1.387)\end{array}$ & $\begin{array}{l}-1.710^{*} \\
(0.863)\end{array}$ & $\begin{array}{l}-1.395^{*} \\
(0.817)\end{array}$ & $\begin{array}{c}-0.622 \\
(0.773)\end{array}$ \\
\hline $\begin{array}{l}\text { Level of } \\
\text { Democracy }\end{array}$ & $\begin{array}{l}-1.416 \\
(0.910)\end{array}$ & $\begin{array}{l}1.154^{* * * *} \\
(0.349)\end{array}$ & $\begin{array}{c}0.503 \\
(0.449)\end{array}$ & $\begin{array}{c}0.447 \\
(0.298)\end{array}$ & $\begin{array}{l}-1.767^{*} \\
(1.019)\end{array}$ & $\begin{array}{c}0.088 \\
(0.360)\end{array}$ & $\begin{array}{c}0.305 \\
(0.369)\end{array}$ & $\begin{array}{l}-0.167 \\
(0.317)\end{array}$ \\
\hline F-Test & 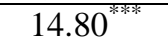 & $4.77^{* * *}$ & 0.83 & 0.11 & $18.08^{* *_{*}^{*}}$ & $2.63^{*}$ & 2.38 & 0.58 \\
\hline Covariates & YES & YES & YES & YES & YES & YES & YES & YES \\
\hline Country FE & NO & YES & YES & YES & NO & NO & NO & NO \\
\hline Mother FE & NO & NO & NO & NO & NO & YES & YES & YES \\
\hline Birth-year FE & NO & NO & YES & NO & NO & NO & YES & NO \\
\hline Trends & NO & NO & NO & YES & NO & NO & NO & YES \\
\hline Sample & Polity $>0$ & Polity $>0$ & $\begin{array}{c}\text { Polity > } \\
0\end{array}$ & $\begin{array}{c}\text { Polity > } \\
0\end{array}$ & $\begin{array}{c}\text { Restricted } \\
\text { - Polity > } \\
0\end{array}$ & $\begin{array}{c}\text { Restricted } \\
\text { - Polity > } \\
0\end{array}$ & $\begin{array}{c}\text { Restricted } \\
\text { - Polity > } \\
0\end{array}$ & $\begin{array}{c}\text { Restricted } \\
\text { - Polity > } \\
0\end{array}$ \\
\hline \# Countries & 48 & 48 & 48 & 48 & 48 & 48 & 48 & 48 \\
\hline \# Observations & 1421049 & 1421049 & 1421049 & 1421049 & 1090431 & 1090431 & 1090431 & 1090431 \\
\hline \multicolumn{9}{|c|}{$\begin{array}{l}\text { Notes: The dependent variable is a binary indicator of infant mortality (multiplied by } 1,000 \text { ). Standard errors } \\
\text { clustered at the country level are reported in parentheses. Statistical significance is indicated as follows: * p }<0.10 \text {, } \\
* * \mathrm{p}<0.05, * * * \mathrm{p}<0.01 \text {. Covariates include the level and growth rate of GDP per person (log terms, constant } 2000 \\
\text { U.S. dollars, adjusted for purchasing power differences across countries), whether the household is located in a rural } \\
\text { area of the country, an indicator for male gender, an indicator for multiple births, and cubic polynomials in mother's } \\
\text { age at the time of birth, years of education, and infant birth order. A constant term is included in each regression but } \\
\text { is not reported. The F-test statistic is for the null hypothesis that the parameters on the election variable and the } \\
\text { interaction variable are both equal to zero. }\end{array}$} \\
\hline
\end{tabular}


Table 3.6 presents results from election year interactions with parliamentary, presidential, and assembly-elected presidential systems of government. Among the different systems of government, countries with parliamentary governments appear to have the strongest election year effects on infant mortality. However, once controlling for unobserved heterogeneity through the use of fixed effects and unobserved year effects and trends in infant mortality, there appears to be little evidence of an electoral cycle in infant mortality rates. 


\section{Table 3.6: Election Year Effects on Infant Mortality Rates - Interactions with System of Government}

\begin{tabular}{|c|c|c|c|c|c|c|c|c|}
\hline Panel A & (1) & (2) & (3) & (4) & (5) & (6) & (7) & (8) \\
\hline Election & $\begin{array}{l}-0.245 \\
(1.819)\end{array}$ & $\begin{array}{l}-1.133^{*} \\
(0.654)\end{array}$ & $\begin{array}{l}-0.028 \\
(0.592)\end{array}$ & $\begin{array}{l}-0.127 \\
(0.761)\end{array}$ & $\begin{array}{l}-0.554 \\
(1.925)\end{array}$ & $\begin{array}{l}-0.399 \\
(0.907)\end{array}$ & $\begin{array}{l}-0.425 \\
(0.890)\end{array}$ & $\begin{array}{l}-0.502 \\
(0.904)\end{array}$ \\
\hline $\begin{array}{l}\text { Election X } \\
\text { Parliamentary }\end{array}$ & $\begin{array}{c}-10.565^{* * *} \\
(2.668)\end{array}$ & $\begin{array}{l}-0.794 \\
(1.454)\end{array}$ & $\begin{array}{l}-0.208 \\
(1.194)\end{array}$ & $\begin{array}{l}-0.398 \\
(0.836)\end{array}$ & $\begin{array}{c}-9.943^{* * *} \\
(2.895)\end{array}$ & $\begin{array}{l}-1.261 \\
(1.333)\end{array}$ & $\begin{array}{l}-0.840 \\
(1.478)\end{array}$ & $\begin{array}{l}-0.694 \\
(1.049)\end{array}$ \\
\hline F-Test & $10.64^{* * * *}$ & $2.77^{*}$ & 0.03 & 1.00 & $9.45^{* * * *}$ & 1.61 & 0.75 & 2.17 \\
\hline \multicolumn{9}{|l|}{ Panel B } \\
\hline Election & $\begin{array}{l}-5.245^{* * *} \\
(2.517)\end{array}$ & $\begin{array}{l}-1.433 \\
(1.038)\end{array}$ & $\begin{array}{l}-0.781 \\
(0.983)\end{array}$ & $\begin{array}{l}-1.228 \\
(1.005)\end{array}$ & $\begin{array}{l}-4.019 \\
(2.954)\end{array}$ & $\begin{array}{l}-1.991 \\
(1.218)\end{array}$ & $\begin{array}{l}-1.870 \\
(1.360)\end{array}$ & $\begin{array}{l}-1.714 \\
(1.208)\end{array}$ \\
\hline $\begin{array}{l}\text { Election X } \\
\text { Presidential }\end{array}$ & $\begin{array}{c}4.542 \\
(4.036)\end{array}$ & $\begin{array}{c}0.225 \\
(1.682)\end{array}$ & $\begin{array}{l}1.125 \\
(1.445)\end{array}$ & $\begin{array}{l}1.636 \\
(1.293)\end{array}$ & $\begin{array}{c}2.327 \\
(4.712)\end{array}$ & $\begin{array}{c}2.092 \\
(1.609)\end{array}$ & $\begin{array}{c}1.986 \\
(1.770)\end{array}$ & $\begin{array}{c}1.672 \\
(1.588)\end{array}$ \\
\hline F-Test & $3.92^{* *}$ & $3.19^{* * *}$ & 0.33 & 0.85 & $3.50^{* *}$ & 1.34 & 0.95 & 1.02 \\
\hline
\end{tabular}

\section{Panel C}

\begin{tabular}{lcccccccc}
\hline Election & $-3.163^{* * *}$ & $-1.379^{*}$ & 0.219 & 0.175 & $-3.764^{* * *}$ & -0.309 & -0.176 & -0.315 \\
& $(1.108)$ & $(0.720)$ & $(0.617)$ & $(0.580)$ & $(1.241)$ & $(0.737)$ & $(0.731)$ & $(0.736)$ \\
Election X & & & & & & & & \\
AEP & 4.451 & 0.499 & -1.600 & -2.224 & $7.453^{*}$ & -2.089 & -2.412 & -2.035 \\
& $(3.457)$ & $(2.230)$ & $(1.859)$ & $(1.866)$ & $(3.742)$ & $(2.326)$ & $(2.440)$ & $(2.418)$ \\
\hline F-Test & & & & & & & & \\
\hline Covariates & YES & YES & YES & YES & YES & YES & YES & YES \\
Country FE & NO & YES & YES & YES & NO & NO & NO & NO \\
Mother FE & NO & NO & NO & NO & NO & YES & YES & YES \\
Birth-year FE & NO & NO & YES & NO & NO & NO & YES & NO \\
Trends & NO & NO & NO & YES & NO & NO & NO & YES \\
Sample & Full & Full & Full & Full & Restricted & Restricted & Restricted & Restricted \\
\# Countries & 69 & 69 & 69 & 69 & 69 & 69 & 69 & 69 \\
\# Obs. & 2522130 & 2522130 & 2522130 & 2522130 & 2088328 & 2088328 & 2088328 & 2088328 \\
\hline
\end{tabular}

Notes: The dependent variable is a binary indicator of infant mortality (multiplied by 1,000). Standard errors clustered at the country level are reported in parentheses. Statistical significance is indicated as follows: $* \mathrm{p}<0.10$, $* * \mathrm{p}<0.05, * * * \mathrm{p}<0.01$. Covariates include the level and growth rate of GDP per person (log terms, constant 2000 U.S. dollars, adjusted for purchasing power differences across countries), whether the household is located in a rural area of the country, an indicator for male gender, an indicator for multiple births, and cubic polynomials in mother's age at the time of birth, years of education, and infant birth order. A constant term is included in each regression but is not reported. Panel A is for the election interaction with a parliamentary system of government, while Panels B and $\mathrm{C}$ are for presidential and assembly-elected presidential systems of government, respectively. The F-test statistic is for the null hypothesis that the parameters on the election variable and the interaction variable are both equal to zero. 


\subsection{Timing of Election Dates and Infant Birth Dates}

Identifying whether changes in infant mortality are related to election cycles may be difficult because of how the timing of elections aligns with infant birth dates and in utero, neonatal, and post-neonatal exposure to election cycles. In the specifications above, I examine whether infant mortality is influenced by whether there is an election in a given year. However, in many cases an infant may be exposed to an electoral cycle not only in the specific year in which she or he was born but also over the span of two different years. Consider that the 1988 legislative election in Haiti was held in November. If there were changes in fiscal policy prior to an election, an infant born in the beginning of 1989 would be exposed to these fluctuations in transfer payments or health service availability in utero. The above specifications would not adequately capture the effects of an election on infant mortality. ${ }^{46}$

In this section, I explore whether differences in the timing of election dates and birth dates influence whether an infant is exposed to electoral cycles. I construct four different variables which capture differences in exposure windows. The first variable (IUE_b) captures in utero exposure to an election prior to the election. The second variable (IUE_a) captures in utero exposure to an election after the election. The third variable (PUE_b) captures neonatal or postneonatal exposure to an election before the election. The last variable (PUE_a) captures neonatal or post-neonatal exposure to an election after the election.

\footnotetext{
${ }^{46}$ As Cutler, Deaton, and Lleras-Muney (2006: 102) note, at the macro-level public health improvements often involve large public works like the development of infrastructure to clean water, the provision of better sanitation facilities, mass vaccination campaigns, and other health interventions. These projects may take place more than one year prior to an election or they may begin very close to the election (i.e. 'quick impact' projects). This suggests that there may not be annual election year effects in infant mortality and that the response could be somewhat lagged. However, in the above specifications, I find very little evidence of lag or lead effects of elections on infant mortality rates. The magnitude for the election-year effects on infant mortality is the largest, followed by the lead effects and lastly by the lag effects.
} 
Each variable is constructed on the basis of the monthly difference between the infant birth month and the election month. For example, if an infant was born in April 2010 and there was an election in July 2010, this observation for the variable $I U E_{-} b$ would be 9 months because the infant would be exposed in utero to an election cycle prior to an election for about 9 months. The measure IUE_a would be 0 months (because the infant was born before the election), the measure $P U E_{-} b$ would be 3 months (because the infant was exposed after being born to the election cycle), and the measure PUE_a would be 9 months (because the infant was exposed after being born to the election cycle after the election had taken place). Table 3.7 provides examples of what these variables would indicate about different exposure windows. 


\section{Table 3.7: Examples of Birth Months and Election Months and Definitions of}

Variables

\begin{tabular}{llrrrrr}
\hline Birth $(\mathbf{B})$ & Election $(\mathbf{E})$ & (B-E) & IUE_b & IUE_a & PUE_b & PUE_a \\
\hline June, 2009 & July, 2010 & -13 & 0 & 0 & 0 & 0 \\
July, 2009 & July, 2010 & -12 & 0 & 0 & 12 & 0 \\
August, 2009 & July, 2010 & -11 & 1 & 0 & 11 & 1 \\
September, 2009 & July, 2010 & -10 & 2 & 0 & 10 & 2 \\
October, 2009 & July, 2010 & -9 & 3 & 0 & 9 & 3 \\
November, 2009 & July, 2010 & -8 & 4 & 0 & 8 & 4 \\
December, 2009 & July, 2010 & -7 & 5 & 0 & 7 & 5 \\
January, 2010 & July, 2010 & -6 & 6 & 0 & 6 & 6 \\
February, 2010 & July, 2010 & -5 & 7 & 0 & 5 & 7 \\
March, 2010 & July, 2010 & -4 & 8 & 0 & 4 & 8 \\
April, 2010 & July, 2010 & -3 & 9 & 0 & 3 & 9 \\
May, 2010 & July, 2010 & -2 & 9 & 0 & 2 & 10 \\
June, 2010 & July, 2010 & -1 & 9 & 0 & 1 & 11 \\
\hline July, 2010 & July, 2010 & $\mathbf{0}$ & $\mathbf{9}$ & $\mathbf{0}$ & $\mathbf{0}$ & 12 \\
\hline August, 2010 & July, 2010 & 1 & 8 & 1 & 0 & 11 \\
September, 2010 & July, 2010 & 2 & 7 & 2 & 0 & 10 \\
October, 2010 & July, 2010 & 3 & 6 & 3 & 0 & 9 \\
November, 2010 & July, 2010 & 4 & 5 & 4 & 0 & 8 \\
December, 2010 & July, 2010 & 5 & 4 & 5 & 0 & 7 \\
January, 2011 & July, 2010 & 6 & 3 & 6 & 0 & 6 \\
February, 2011 & July, 2010 & 7 & 2 & 7 & 0 & 5 \\
March, 2011 & July, 2010 & 8 & 1 & 8 & 0 & 4 \\
April, 2011 & July, 2010 & 9 & 0 & 9 & 0 & 3 \\
May, 2011 & July, 2010 & 10 & 0 & 9 & 0 & 2 \\
June, 2011 & July, 2010 & 11 & 0 & 9 & 0 & 1 \\
July, 2011 & July, 2010 & 12 & 0 & 9 & 0 & 0 \\
August, 2011 & July, 2010 & 13 & 0 & 0 & 0 & 0 \\
\hline & & & & & & \\
\end{tabular}

Because the differences between birth months and election months may become quite large (i.e. an infant born in July 2007 could be exposed in utero to an election in July 2010), I taper the windows in which infants are exposed to elections. For instance, instead of allowing in utero exposure before an election to reach a high level, I begin to taper this number down. An infant born in April 2010 when there is a July 2010 election would have 9 months of in utero exposure prior to the election, but an infant born in March 2010 would have 8 months of in utero 
exposure. In essence, I cap the exposure windows to one year before and one year after the election cycle in order to constrain the effects of elections on infant mortality for a specific time period. There are likely many ways to create these variables for capturing exposure to elections and the evidence in this section should be seen as suggestive but not conclusive. ${ }^{47}$

I now seek to estimate the parameters of the following linear probability model:

$$
\text { mortality }_{\text {ihmcy }}=\iota_{h}+\sigma_{y}+\beta \mathrm{IUE}_{\mathrm{i}}+\mathrm{Z}_{\mathrm{ih}}^{\prime} \gamma+\mathrm{X}_{\mathrm{cy}}^{\prime} \delta+\varepsilon_{\mathrm{ihmcy}}
$$

$\beta$ is now the impact of an additional month of in utero exposure to an election cycle for infant $i$ on infant mortality. In the regression results reported below, I control for either country fixed effects or mother fixed effects and cluster the observations of error term at the country level to control for arbitrary correlation among infant births at the country level. I further include birthyear/cohort fixed effects or country-specific linear trends in infant mortality to control for time dimensions and trends in infant mortality. I report only estimates of in utero exposure to electoral cycles. All the other results for neonatal or post-neonatal exposure were generally insignificant, though still relevant for empirical testing. ${ }^{48}$

The results for regressions where in utero exposure to election cycles is the key explanatory variable are very similar to those presented in Section 5. The strongest evidence for electoral cycles in infant mortality rates is found when the sample is restricted to only those countries with Polity scores strictly greater than zero. Column 7 in Table 3.9 presents regression results from mother fixed effects panel regressions with birth year fixed effects. The coefficient estimates suggest that an additional month of in utero exposure to an election cycle would be associated with an increase in the infant mortality rate if elections were completely non-

\footnotetext{
${ }^{47}$ There are some limitations to this strategy. For instance, I examine an in utero exposure window of 9 months. This is likely to be a reasonable approximation for most pregnancies.

${ }^{48}$ Results for the other exposure windows are available from the author upon request.
} 
competitive. Elections would have to be nearly fully competitive for infant mortality rates to fall prior to an election. The impact of democracy appears to make evidence for election year cyclicality in infant mortality disappear, even when restricting the sample to those countries with relatively young democracies. All results for different specifications are presented in Table 3.8, Table 3.9, Table 3.10, and Table 3.11. 


\section{Table 3.8: In Utero Exposure to the Election Cycle}

\section{Panel A: Months of In Utero Exposure to Elections}

\begin{tabular}{lcccccccc}
\hline & $(1)$ & $(2)$ & $(3)$ & $(4)$ & $(5)$ & $(6)$ & $(7)$ & $(8)$ \\
\hline IUE Before & $-0.369^{* *}$ & $-0.183^{*}$ & -0.014 & -0.038 & $-0.380^{* *}$ & -0.048 & -0.028 & -0.042 \\
& $(0.142)$ & $(0.094)$ & $(0.093)$ & $(0.088)$ & $(0.150)$ & $(0.094)$ & $(0.093)$ & $(0.095)$ \\
& & & & & & & & \\
\hline Covariates & YES & YES & YES & YES & YES & YES & YES & YES \\
Country FE & NO & YES & YES & YES & NO & NO & NO & NO \\
Mother FE & NO & NO & NO & NO & NO & YES & YES & YES \\
Birth-year FE & NO & NO & YES & NO & NO & NO & YES & NO \\
Trends & NO & NO & NO & YES & NO & NO & NO & YES \\
Sample & Full & Full & Full & Full & Restricted & Restricted & Restricted & Restricted \\
\# Countries & 68 & 68 & 68 & 68 & 68 & 68 & 68 & 68 \\
\# Observations & 2551099 & 2551099 & 2551099 & 2551099 & 2119752 & 2119752 & 2119752 & 2119752 \\
\hline
\end{tabular}

Panel B: Controlling for Polity Score

\begin{tabular}{lcccccccc}
\hline & $(1)$ & $(2)$ & $(3)$ & $(4)$ & $(5)$ & $(6)$ & $(7)$ & $(8)$ \\
\hline IUE Before & $-0.233^{* *}$ & -0.090 & -0.013 & -0.041 & $-0.242^{*}$ & -0.028 & -0.012 & -0.037 \\
& $(0.115)$ & $(0.080)$ & $(0.088)$ & $(0.085)$ & $(0.128)$ & $(0.095)$ & $(0.093)$ & $(0.095)$ \\
& & & & & & & \\
& $-0.657^{* *}$ & $-1.034^{* * *}$ & -0.042 & 0.068 & $-0.679^{* *}$ & $-0.486^{* * *}$ & $-0.355^{* *}$ & -0.098 \\
Level of & $(0.274)$ & $(0.309)$ & $(0.272)$ & $(0.180)$ & $(0.284)$ & $(0.166)$ & $(0.170)$ & $(0.138)$ \\
Democracy & YES & YES & YES & YES & YES & YES & YES & YES \\
\hline Covariates & NO & YES & YES & YES & NO & NO & NO & NO \\
Country FE & NO & NO & NO & NO & NO & YES & YES & YES \\
Mother FE & NO & NO & YES & NO & NO & NO & YES & NO \\
Birth-year FE & NO & NO & NO & YES & NO & NO & NO & YES \\
Trends & Full & Full & Full & Full & Restricted & Restricted & Restricted & Restricted \\
Sample & 68 & 68 & 68 & 68 & 68 & 68 & 68 & 68 \\
\# Countries & 2542586 & 2542586 & 2542586 & 2542586 & 2111089 & 2111089 & 2111089 & 2111089 \\
\# Observations & & & & & & & & \\
\hline
\end{tabular}

Notes: The dependent variable is a binary indicator of infant mortality (multiplied by 1,000). Standard errors clustered at the country level are reported in parentheses. Statistical significance is indicated as follows: $* \mathrm{p}<0.10$, $* * \mathrm{p}<0.05,{ }^{* * *} \mathrm{p}<0.01$. Covariates include the level and growth rate of GDP per person (log terms, constant 2000 U.S. dollars, adjusted for purchasing power differences across countries), whether the household is located in a rural area of the country, an indicator for male gender, an indicator for multiple births, and cubic polynomials in mother's age at the time of birth, years of education, and infant birth order. A constant term is included in each regression but is not reported. 


\section{Table 3.9: In Utero Exposure to the Election Cycle}

\begin{tabular}{|c|c|c|c|c|c|c|c|c|}
\hline \multicolumn{9}{|c|}{ Panel A: mteraction with Electoral competitiveness } \\
\hline & (1) & (2) & (3) & (4) & (5) & (6) & $(7)$ & (8) \\
\hline IUE Before & $\begin{array}{l}1.682^{* *} \\
(0.715)\end{array}$ & $\begin{array}{l}1.216^{* * * *} \\
(0.395)\end{array}$ & $\begin{array}{c}0.568 \\
(0.427)\end{array}$ & $\begin{array}{c}0.243 \\
(0.339)\end{array}$ & $\begin{array}{l}1.736^{* *} \\
(0.807)\end{array}$ & $\begin{array}{c}0.393 \\
(0.269)\end{array}$ & $\begin{array}{c}0.292 \\
(0.285)\end{array}$ & $\begin{array}{c}0.315 \\
(0.265)\end{array}$ \\
\hline IUE X Comp & $\begin{array}{c}-0.356^{* * * *} \\
(0.128)\end{array}$ & $\begin{array}{c}-0.249^{* * *} \\
(0.072)\end{array}$ & $\begin{array}{l}-0.101 \\
(0.076)\end{array}$ & $\begin{array}{l}-0.045 \\
(0.061)\end{array}$ & $\begin{array}{l}-0.372^{* *} \\
(0.145)\end{array}$ & $\begin{array}{l}-0.080 \\
(0.051)\end{array}$ & $\begin{array}{l}-0.059 \\
(0.054)\end{array}$ & $\begin{array}{l}-0.064 \\
(0.051)\end{array}$ \\
\hline F-Test & $5.93^{* * * *}$ & $6.15^{* * * *}$ & 0.90 & 0.27 & $5.30^{* * * *}$ & 1.23 & 0.62 & 0.79 \\
\hline Covariates & YES & YES & YES & YES & YES & YES & YES & YES \\
\hline Country FE & NO & YES & YES & YES & NO & NO & NO & NO \\
\hline Mother FE & NO & NO & NO & NO & NO & YES & YES & YES \\
\hline Birth-year FE & NO & NO & YES & NO & NO & NO & YES & NO \\
\hline Trends & NO & NO & NO & YES & NO & NO & NO & YES \\
\hline Sample & Full & Full & Full & Full & Restricted & Restricted & Restricted & Restricted \\
\hline \# Countries & 68 & & 68 & 68 & 68 & 68 & 68 & 68 \\
\hline \# Obs. & 2517068 & 2517068 & 2517068 & 2517068 & 2084526 & 2084526 & 2084526 & 2084526 \\
\hline \multicolumn{9}{|c|}{ Panel B: Controlling for Polity Score } \\
\hline & (1) & $(2)$ & (3) & (4) & $(5)$ & (6) & (7) & (8) \\
\hline IUE Before & $\begin{array}{c}0.150 \\
(0.271)\end{array}$ & $\begin{array}{c}0.157 \\
(0.268)\end{array}$ & $\begin{array}{c}0.128 \\
(0.272)\end{array}$ & $\begin{array}{l}-0.008 \\
(0.238)\end{array}$ & $\begin{array}{c}0.162 \\
(0.295)\end{array}$ & $\begin{array}{c}0.113 \\
(0.201)\end{array}$ & $\begin{array}{c}0.108 \\
(0.189)\end{array}$ & $\begin{array}{c}0.107 \\
(0.199)\end{array}$ \\
\hline IUE x Comp & $\begin{array}{l}-0.079 \\
(0.053)\end{array}$ & $\begin{array}{l}-0.051 \\
(0.046)\end{array}$ & $\begin{array}{l}-0.030 \\
(0.046)\end{array}$ & $\begin{array}{l}-0.007 \\
(0.042)\end{array}$ & $\begin{array}{l}-0.084 \\
(0.058)\end{array}$ & $\begin{array}{l}-0.030 \\
(0.037)\end{array}$ & $\begin{array}{l}-0.026 \\
(0.035)\end{array}$ & $\begin{array}{l}-0.031 \\
(0.037)\end{array}$ \\
\hline $\begin{array}{l}\text { Level of } \\
\text { Democracy }\end{array}$ & $\begin{array}{c}-0.644^{* *} \\
(0.275)\end{array}$ & $\begin{array}{c}-1.022^{* * * *} \\
(0.312)\end{array}$ & $\begin{array}{l}-0.036 \\
(0.273)\end{array}$ & $\begin{array}{c}0.069 \\
(0.181)\end{array}$ & $\begin{array}{c}-0.664^{* * *} \\
(0.285)\end{array}$ & $\begin{array}{c}-0.483^{* * * *} \\
(0.166)\end{array}$ & $\begin{array}{c}-0.354^{* * *} \\
(0.171)\end{array}$ & $\begin{array}{l}-0.095 \\
(0.137)\end{array}$ \\
\hline F-Test & $2.90^{*}$ & 2.32 & 0.36 & 0.16 & $2.48^{*}$ & 0.38 & 0.27 & 0.42 \\
\hline Covariates & YES & YES & YES & YES & YES & YES & YES & YES \\
\hline Country FE & NO & YES & YES & YES & NO & NO & NO & NO \\
\hline Mother FE & NO & NO & NO & NO & NO & YES & YES & YES \\
\hline Birth-year FE & NO & NO & YES & $\mathrm{NO}$ & NO & NO & YES & NO \\
\hline Trends & NO & NO & NO & YES & NO & NO & NO & YES \\
\hline Sample & Full & Full & Full & Full & Restricted & Restricted & Restricted & Restricted \\
\hline \# Countries & 68 & 68 & 68 & 68 & 68 & 68 & 68 & 68 \\
\hline \# Observations & 2539420 & 2539420 & 2539420 & 2539420 & 2108799 & 2108799 & 2108799 & 2108799 \\
\hline
\end{tabular}

Notes: The dependent variable is a binary indicator of infant mortality (multiplied by 1,000 ). Standard errors clustered at the country level are reported in parentheses. Statistical significance is indicated as follows: $* \mathrm{p}<0.10$, ** $\mathrm{p}<0.05$, *** $\mathrm{p}<0.01$. Covariates include the level and growth rate of GDP per person (log terms, constant 2000 U.S. dollars, adjusted for purchasing power differences across countries), whether the household is located in a rural area of the country, an indicator for male gender, an indicator for multiple births, and cubic polynomials in mother's age at the time of birth, years of education, and infant birth order. A constant term is included in each regression but is not reported. The F-test statistic is for the null hypothesis that the parameters on the election variable and the interaction variable are both equal to zero. 


\section{Table 3.10: In Utero Exposure to the Election Cycle - Interaction with Electoral Competitiveness}

\section{Panel A: Only for Country-Year Observations with Polity $>0$}

\begin{tabular}{lcccccccc}
\hline & $(1)$ & $(2)$ & $(3)$ & $(4)$ & $(5)$ & $(6)$ & $(7)$ & $(8)$ \\
\hline IUE Before & $4.444^{* * * *}$ & $2.138^{* * *}$ & 1.177 & 0.216 & $7.285^{* * *}$ & $2.430^{* * *}$ & $2.285^{* * * *}$ & 1.211 \\
& $(0.974)$ & $(0.769)$ & $(0.863)$ & $(0.899)$ & $(1.418)$ & $(0.767)$ & $(0.758)$ & $(0.762)$ \\
& & & & & & & \\
& & & & & & \\
& & & & & \\
IUE X Comp & $-0.736^{* * *}$ & $-0.344^{* * *}$ & -0.181 & -0.033 & $-1.175^{* * *}$ & $-0.354^{* * *}$ & $-0.323^{* *}$ & -0.174 \\
& $(0.148)$ & $(0.115)$ & $(0.138)$ & $(0.141)$ & $(0.206)$ & $(0.120)$ & $(0.120)$ & $(0.119)$ \\
& & & & & & & & \\
\hline F-Test & $13.36^{* * * *}$ & $5.61^{* * *}$ & 0.97 & 0.03 & $19.61^{* * *}$ & $5.72^{* * *}$ & $5.94^{* * *}$ & 1.49 \\
\hline Covariates & YES & YES & YES & YES & YES & YES & YES & YES \\
Country FE & NO & YES & YES & YES & NO & NO & NO & NO \\
Mother FE & NO & NO & NO & NO & NO & YES & YES & YES \\
Birth-year FE & NO & NO & YES & NO & NO & NO & YES & NO \\
Trends & NO & NO & NO & YES & NO & NO & NO & YES \\
Sample & Polity $>0$ & Polity $>0$ & Polity $>0$ & Polity $>0$ & Restricted & Restricted & Restricted & Restricted - \\
\# Countries & 48 & 48 & 48 & 48 & - Polity $>0$ & - Polity $>0$ & - Polity $>0$ & Polity $>0$ \\
\# Obs. & 1421049 & 1421049 & 1421049 & 1421049 & 1090431 & 1090431 & 1090431 & 1090431 \\
\hline
\end{tabular}

Panel B: Controlling for Polity Score

\begin{tabular}{lcccccccc}
\hline & $(1)$ & $(2)$ & $(3)$ & $(4)$ & $(5)$ & $(6)$ & $(7)$ & $(8)$ \\
\hline IUE Before & 0.532 & 0.364 & 0.357 & 0.178 & 0.638 & 0.430 & 0.514 & 0.284 \\
& $(0.432)$ & $(0.321)$ & $(0.291)$ & $(0.298)$ & $(0.476)$ & $(0.371)$ & $(0.307)$ & $(0.342)$ \\
& & & & & & & & \\
IUE x Comp & $-0.135^{*}$ & -0.082 & -0.065 & -0.033 & $-0.165^{*}$ & -0.055 & -0.060 & -0.038 \\
& $(0.078)$ & $(0.055)$ & $(0.055)$ & $(0.051)$ & $(0.090)$ & $(0.058)$ & $(0.052)$ & $(0.053)$ \\
& & & & & & & & \\
Level of & -1.562 & $1.208^{* * *}$ & 0.536 & 0.453 & $-1.968^{*}$ & 0.132 & 0.367 & -0.155 \\
Democracy & $(0.957)$ & $(0.338)$ & $(0.445)$ & $(0.300)$ & $(1.079)$ & $(0.363)$ & $(0.368)$ & $(0.316)$ \\
\hline F-Test & 2.06 & 1.80 & 0.76 & 0.21 & 2.06 & 0.73 & 1.72 & 0.36 \\
\hline Covariates & YES & YES & YES & YES & YES & YES & YES & YES \\
Country FE & NO & YES & YES & YES & NO & NO & NO & NO \\
Mother FE & NO & NO & NO & NO & NO & YES & YES & YES \\
Birth-year FE & NO & NO & YES & NO & NO & NO & YES & NO \\
Trends & NO & NO & NO & YES & NO & NO & NO & YES \\
Sample & Polity $>$ & Polity $>$ & Polity $>$ & Polity > & Restricted & Restricted & Restricted & Restricted \\
\# Countries & 0 & 0 & 0 & 0 & - Polity > & - Polity >0 & - Polity > 0 & - Polity > 0 \\
\# Observation & 48 & 48 & 48 & 48 & 48 & 48 & 48 & 48 \\
& 1421049 & 1421049 & 1421049 & 1421049 & 1090431 & 1090431 & 1090431 & 1090431 \\
\hline
\end{tabular}

Notes: The dependent variable is a binary indicator of infant mortality (multiplied by 1,000). Standard errors clustered at the country level are reported in parentheses. Statistical significance is indicated as follows: $* p<0.10$, $* * \mathrm{p}<0.05, * * * \mathrm{p}<0.01$. Covariates include the level and growth rate of GDP per person (log terms, constant 2000 U.S. dollars, adjusted for purchasing power differences across countries), whether the household is located in a rural area of the country, an indicator for male gender, an indicator for multiple births, and cubic polynomials in mother's age at the time of birth, years of education, and infant birth order. A constant term is included in each regression but is not reported. The F-test statistic is for the null hypothesis that the parameters on the election variable and the interaction variable are both equal to zero. 


\section{Table 3.11: In Utero Exposure to the Election Cycle - Interactions with Systems of Government}

\begin{tabular}{lcccccccc} 
Panel A & $(1)$ & $(2)$ & $(3)$ & $(4)$ & $(5)$ & $(6)$ & $(7)$ & $(8)$ \\
\hline Election & 0.042 & -0.110 & 0.048 & 0.032 & -0.004 & 0.008 & 0.005 & -0.005 \\
& $(0.258)$ & $(0.115)$ & $(0.106)$ & $(0.101)$ & $(0.276)$ & $(0.115)$ & $(0.112)$ & $(0.113)$ \\
& & & & & & \\
Election X & $-1.641^{* * *}$ & -0.220 & -0.175 & -0.169 & $-1.536^{* * *}$ & -0.228 & -0.165 & -0.137 \\
Parliamentary & $(0.426)$ & $(0.252)$ & $(0.209)$ & $(0.128)$ & $(0.457)$ & $(0.176)$ & $(0.194)$ & $(0.149)$ \\
& & & & & & & & \\
\hline F-Test & $9.91^{* * *}$ & 2.07 & 0.36 & 1.76 & $8.72^{* * *}$ & 1.53 & 0.59 & 1.04 \\
\hline
\end{tabular}

Panel B

\begin{tabular}{lcccccccc}
\hline Election & $-0.748^{*}$ & -0.181 & -0.121 & $-0.204^{*}$ & -0.565 & $-0.281^{*}$ & $-0.259^{*}$ & $-0.250^{*}$ \\
& $(0.414)$ & $(0.152)$ & $(0.123)$ & $(0.121)$ & $(0.477)$ & $(0.146)$ & $(0.150)$ & $(0.145)$ \\
& & & & & & & & \\
Election X & 0.714 & 0.042 & 0.215 & $0.324^{*}$ & 0.387 & $0.381^{*}$ & $0.361^{*}$ & $0.340^{*}$ \\
Presidential & $(0.638)$ & $(0.265)$ & $(0.205)$ & $(0.169)$ & $(0.734)$ & $(0.196)$ & $(0.203)$ & $(0.192)$ \\
& & & & & & & & \\
\hline F-Test & $2.91^{*}$ & 1.94 & 0.59 & 1.89 & $2.59^{*}$ & 2.07 & 1.68 & 1.67 \\
\hline
\end{tabular}

Panel C

\begin{tabular}{lcccccccc}
\hline Election & $-0.426^{* *}$ & -0.184 & 0.044 & 0.055 & $-0.509^{* * * *}$ & 0.023 & 0.043 & 0.033 \\
& $(0.165)$ & $(0.114)$ & $(0.105)$ & $(0.087)$ & $(0.180)$ & $(0.095)$ & $(0.094)$ & $(0.095)$ \\
& & & & & & & & \\
Election X & 0.710 & 0.171 & -0.166 & -0.339 & $1.144^{* *}$ & -0.384 & -0.428 & -0.422 \\
AEP & $(0.501)$ & $(0.297)$ & $(0.233)$ & $(0.254)$ & $(0.536)$ & $(0.274)$ & $(0.264)$ & $(0.281)$ \\
& & & & & & & & \\
\hline F-Test & $3.36^{* *}$ & 1.51 & 0.25 & 0.89 & $4.17^{* *}$ & 1.07 & 1.35 & 1.17 \\
\hline Covariates & YES & YES & YES & YES & YES & YES & YES & YES \\
Country FE & NO & YES & YES & YES & NO & NO & NO & NO \\
Mother FE & NO & NO & NO & NO & NO & YES & YES & YES \\
Birth-year FE & NO & NO & YES & NO & NO & NO & YES & NO \\
Trends & NO & NO & NO & YES & NO & NO & NO & YES \\
Sample & Full & Full & Full & Full & Restricted & Restricted & Restricted & Restricted \\
\# Countries & 68 & 68 & 68 & 68 & 68 & 68 & 68 & 68 \\
\# Obs. & 2517068 & 2517068 & 2517068 & 2517068 & 2084526 & 2084526 & 2084526 & 2084526 \\
\hline
\end{tabular}

Notes: The dependent variable is a binary indicator of infant mortality (multiplied by 1,000). Standard errors clustered at the country level are reported in parentheses. Statistical significance is indicated as follows: $* \mathrm{p}<0.10$, $* * \mathrm{p}<0.05$, *** $\mathrm{p}<0.01$. Covariates include the level and growth rate of GDP per person (log terms, constant 2000 U.S. dollars, adjusted for purchasing power differences across countries), whether the household is located in a rural area of the country, an indicator for male gender, an indicator for multiple births, and cubic polynomials in mother's age at the time of birth, years of education, and infant birth order. A constant term is included in each regression but is not reported. Panel A is for the election interaction with a parliamentary system of government, while Panels B and $\mathrm{C}$ are for presidential and assembly-elected presidential systems of government, respectively. The F-test statistic is for the null hypothesis that the parameters on the election variable and the interaction variable are both equal to zero. 


\subsection{Conclusion}

Across the developing world, infant mortality rates exhibit a remarkable amount of variation, both across and within countries and over time. Most studies to date have focused on economic, environmental, and disease ecological explanations for fluctuations in infant mortality rates, yet few studies have addressed whether there are systematic variations in infant mortality rates as a result of political economy factors.

This paper expands the literature on 'election economics' by testing whether electoral cycles shape individual-level health outcomes across the developing world. I find only limited evidence for electoral cyclicality in infant mortality rates. The strongest evidence is for those countries that have relatively higher levels of democracy but also for those countries with relatively younger democracies. In matching election dates and infant birth dates, I find suggestive but limited evidence that in utero exposure to election cycles is associated with reductions in infant mortality rates. More research (and data) is needed to evaluate the potential transmission mechanisms from electoral cycles to infant mortality. 


\section{Chapter 4}

\section{Economic Reconstruction Amidst Conflict - Insights from Afghanistan and Iraq}

\subsection{Introduction}

Economic reconstruction involves fundamentally transforming a society's economic institutions in order to foster growth and development as well as to resolve perceived economic and social problems. In many conflict and post-conflict settings, infrastructure has been destroyed and roads, power stations, water pipelines, hospitals, schools, and sewerage facilities must be completely rebuilt. In addition to the destruction of physical capital, human capital and social capital are seriously damaged by conflict. The economic reconstruction process as a whole therefore involves rebuilding infrastructure, restoring physical, social, and human capital, and restructuring fiscal, monetary, and trade policies in order to allow for a robust private sector to emerge.

Historically, economic reconstruction has taken place following the end of conflict, as in the cases of post-World War II Japan and West Germany. However, in more recent foreign interventions such as Afghanistan and Iraq, economic reconstruction has been viewed as part of a broader strategy to "win the hearts and minds" of domestic political authorities, citizens, and insurgents in order to end conflict. Economic reconstruction in such contexts has proven to be highly complex and uncertain.

There have been many successful reconstruction projects and reforms in both Afghanistan and Iraq - the rebuilding of schools, hospitals, and infrastructure has taken place despite the presence of violent insurgencies. School enrollment has skyrocketed in Afghanistan, 
and girls, who were previously unable to attend school under Taliban rule, now comprise a third of the country's education enrollment (Isby 2010; Miliband 2010).

However, there have also been significant setbacks due to ongoing conflict in addition to a variety of other factors discussed in this paper. For instance, insurgents often target aid workers as well as infrastructure projects that have been administered by foreign reconstruction authorities. In Iraq, for instance, insurgents often targeted oil pipelines and other major infrastructure programs administered by the U.S. and its allies. Gul Agha Ahmadi, a spokesman for the Ministry of Education in Afghanistan, has reported that around 60 schools have been burned down in 2010 at the time of writing (Norland 2010).

Despite the importance of economic reconstruction, the topic has received relatively little attention from the academic community. As Del Castillo (2008: 19-20) notes, “...economic reconstruction has been a much-neglected aspect of the extensive and fast-growing literature on war-to-peace transitions." This is especially the case in the context of ongoing insurgencies in Afghanistan and Iraq. The purpose of this paper is to fill this gap in the literature by analyzing economic reconstruction amidst ongoing conflict in Afghanistan and Iraq.

Our analysis is situated in multiple strands of related literature. Since post-conflict reconstruction typically involves social and economic change in the broadest sense, some of the literature on the topic assesses the multiple facets of rebuilding a society's economic, legal, political, and social institutions in the wake of conflict (see Kumar 1998; Ikenberry 2001; Bastian, Sunil, and Robin Luckham 2003; Chimni 2003; Demekas, McHugh, and Kosma 2003; Williams 2003; Coyne 2008; Coyne and Boettke 2009). Others provide historical contexts for reconstruction activities or critically assess international donors' plans for development in postconflict societies (Montgomery and Rondinelli 2004). Another strand of literature on 
reconstruction has focused more specifically on the economic aspects of reconstruction, such as fiscal and monetary policies or infrastructural development. For example, the contributors to the volume edited by Boyce (1996) explore the economic policies conducive to peace based on reconstruction experiences in El Salvador. Boyce (2002) highlights the role of conditional aid in encouraging domestic investment in peace. Adam et al. (2008) analyze the role played by aid in monetary reconstruction. Del Castillo (2008) emphasizes that post-conflict economic reconstruction is a multi-pronged challenge which involves standard issues of socio-economic development as well as the need to consolidate peace and provide basic services to citizens. Collier (2009) analyzes whether post-conflict economic policies should be distinctive from those adopted in equally poor, but peaceful, countries.

Our analysis differs from, and contributes to, this existing literature in several important ways. First, in contrast to the first category of literature on post-conflict reconstruction, we focus on one specific aspect of reconstruction-reforming economic institutions and policies. Second, the existing literature tends to neglect the different incentives and constraints facing the array of actors involved in economic reconstruction-domestic and foreign policymakers, aid workers and development experts, members of the military, diplomats, etc. Currently, much of the literature is focused on identifying the challenges involved in economic reconstruction, as well as the ideal policies necessary to address these challenges. While identifying challenges and solutions is clearly important, it is only part of the story. Also important is the ability to actually design and implement policies in a way that effectively address the challenges of economic reconstruction. Third, our analysis focuses on economic reconstruction in the context of ongoing insurgencies. Much of the existing literature is focused on economic reconstruction efforts that take place in post-conflict settings and therefore assumes that economic reconstruction takes 
place in an environment where conflict is minimal or has altogether ended. However, as the insurgencies in Afghanistan and Iraq illustrate, this has not recently been the case. Given our focus on economic reconstruction amidst ongoing conflict and insurgency, our analysis places particular emphasis on the role played by military officials, in addition to other key players involved in economic reconstruction. Neglecting how ongoing conflict and insurgency influences economic reconstruction efforts overlooks potential sources of tension between various, and often competing, goals-e.g., military, diplomatic, peace building, economic reconstruction and development, etc. This is crucial given that economic reconstruction rarely takes place in isolation and is typically part of a broader policy agenda which includes state building and peace building.

To date, these aspects of economic reconstruction have not received the attention they deserve. Our central argument is that the array of actors involved in economic reconstruction efforts face different incentives and constraints which influence their ability to effectively design and implement economic reforms and reconstruction projects. These constraints exist in all economic reconstruction efforts, but become increasingly complex and relevant in cases where conflict is still ongoing such as Afghanistan and Iraq.

We identify four main 'reconstruction traps' which often hamper efforts to rebuild economies amidst conflict. These traps include: (1) the credible commitment trap, (2) the knowledge trap, (3) the political economy trap, and (4) the bureaucracy trap. In order to illustrate the dynamics of each trap, we draw from a variety of experiences in Afghanistan and Iraq. Avoiding these traps is critical for success in economic reconstruction so we discuss potential strategies for avoiding them in current and future reconstruction efforts. 
In what follows, we dedicate a section to each of these four economic reconstruction traps. We discuss the dynamics of each trap and also consider strategies to avoid the pitfalls created by these traps. The final section concludes with the implications of our analysis.

\subsection{The Credible Commitment Trap}

The credible commitment problem can be understood as follows. Without a binding and credible commitment to economic reforms (e.g. reconstruction projects or fundamental changes to fiscal, monetary trade, or regulatory policies), some reconstruction officials may have the incentive to change course in the future. ${ }^{49}$ If this is the case, then economic reforms will be time inconsistent. Time inconsistency of reforms results in regime uncertainty - the instability of rules and institutions over time-which threatens the likelihood of success in broader efforts to end conflict and reconstruct the economies of war-torn societies.

The credible commitment problem has been identified as one of the most significant impediments to effective post-conflict economic reconstruction (Coyne and Boettke, 2009; Flores and Nooruddin, 2009). As we discuss below, the credible commitment trap may emerge because the operational environment is inherently dynamic. The inability to make a credible commitment may also be a function of the complexity caused by the interactions of multiple reformers (e.g. U.S. military officials, Afghan national government officials, aid organization officials, local warlords, etc.), some of which may perceive that they must change their strategies given new developments or have competing objectives.

\footnotetext{
${ }^{49}$ As Acemoglu and Robinson (2006: 193) explain, credibility becomes an issue when there is a disjoint between those holding power and the beneficiaries of announced reforms because those holding power may renege on their vocalized intent in later periods and beneficiaries have limited recourse when they do so.
} 


\subsubsection{The Credible Commitment Problem}

To illustrate the dynamics of the credible commitment trap, consider that regime uncertainty has hindered economic reconstruction efforts in Iraq and Afghanistan. As oft-cited examples, in Iraq, the initial governing authority - the Coalition Provisional Authority (CPA) - reneged on an economic reform regarding the privatization of state owned enterprises (SOEs) after seeing how the initial privatization subsequently affected unemployment and the economy in general. While essentially unintended, this reform reversal likely led many Iraqi citizens and local political authorities to question the legitimacy of future economic reforms announced by the CPA.

In the case of Afghanistan, constant changes to the general policy environment by the military occupiers have contributed to fundamental and widespread uncertainty on the part of Afghan leaders and citizens. As Stewart (2010) explains:

We [the U.S. and its allies] armed militias in 2001, disarmed them through a demobilization program in 2003, and rearmed them again in 2006 as community defense forces. We allowed local autonomy in 2001, pushed for a strong central government in 2003, and returned to decentralization in 2006. First we tolerated opium crops; then we proposed to eradicate them through aerial spraying; now we expect to live with opium production for decades.

This lack of commitment to a particular policy framework, while necessary for a dynamic and flexible approach to economic reconstruction amidst conflict, can inadvertently hinder the credibility of future policies of foreign governments and international organizations. ${ }^{50}$ Such drastic changes in policy imply that both the Afghan national government and foreign reformers will be seen as lacking commitment to a continuous policy regime. In the context of an ongoing insurgency, credible commitments to reforms become incredibly difficult because there are multiple reconstruction officials and domestic political authorities vested with decision making

\footnotetext{
${ }^{50}$ As described by Stewart (2010), "Frustrated by a lack of progress, the U.S. and its allies have oscillated giddily between contradictory policies [in Afghanistan]. The British government that once championed more generous budgetary support for the Kabul government now portrays it as corrupt, semi-criminal, ineffective, and illegitimate."
} 
power. External policymakers and military occupiers can be construed as one set of reformers while domestic political leaders, local power brokers, and insurgents at the national and local levels are another. The dispersion of policy decision making power among different reconstruction officials and political authorities makes avoiding the credible commitment trap that much more difficult because determining and establishing the appropriate incentives for reformers at each level is an extremely difficult task. Regime uncertainty on the part of one group of policy makers can make credible commitments to reforms by other groups of policy makers tenuous.

Along with fragmentation in policy decision making power, social fragmentation also complicates the ability of different reconstruction officials to make credible commitments to a specific policy regime. Consider that in Iraq, successful economic reforms needed to satisfy members of Iraq's major ethnic groups — the Arabs and the Kurds—and religious groups—Shi'a and Sunni Muslims. In reality, the situation is even more complex given that there are various intra-group factions in the broader Sunni and Shi'a groups in Iraq at both the national and local levels (Fearon 2007). This is also the case with Afghanistan's ethnic groups with ethnic Pashtuns, Tajiks, Hazaras, Uzbeks, and many other groups all comprising power in Afghan governance (national and local). Finding a policy consensus to which the various reformers can commit under ethnic fractionalization or polarization is especially daunting in these situations, as the commitment to a specific policy may isolate a particular ethnic group or renege on a promise to these individuals.

An existing literature on reform, peace building, and aid effectiveness highlights the importance of domestic policy ownership (see Sobhan 2002). As put by World Bank President 
Robert Zoellick (2008), "local ownership is key to achieving legitimacy and effectiveness." The underlying idea is that without domestic buy-in and ownership, economic reforms will not stick and operate in the desired manner. Local policy ownership also obviates the need to coordinate the efforts of multiple reconstruction officials, some who have competing objectives and visions for economic reconstruction.

Yet with policy decision making power dispersed among numerous domestic policy makers, policy ownership is still highly complex. With competing objectives even among different domestic political authorities, allowing for policy ownership implies that some economic reforms will be adopted that will be discordant with others. The problem is further exacerbated by ongoing conflict because reconstruction officials and citizens cannot be sure about what the distribution of power will be when conflict does end. This further contributes to the uncertainty regarding who the true 'reformers' are which weakens the credibility of announced reforms at various levels.

As this illustrates, the credible commitment problem poses a great deal of complexity and uncertainty for reconstruction officials. Historically, credible commitments in Afghanistan have been difficult. The problem facing foreign reconstruction officials is twofold. First, they must determine what exactly domestic legitimacy entails. Is legitimacy a function of the ability to make a credible commitment or is it moreover a problem of misaligned expectations among local citizens and political authorities? Second, foreign reformers have to allow for domestic ownership over economic reforms. This is hard enough in post-conflict settings, but ongoing conflict makes the challenge that much more difficult.

While the establishment of legitimacy is seen as the main objective of counterinsurgency (COIN) and reconstruction operations by the U.S. military (U.S. Army/Marine Corps, 2007: 1- 
21; U.S. Department of the Army, 2008: 1-7.), supporting the legitimacy of host nations and promoting domestic policy ownership can be a difficult task. In order for domestic ownership to be established, domestic political authorities and citizens alike must have the incentives to coalesce around specific economic reforms. Consider that the National Solidarity Program, a development program implemented by the Afghan Ministry of Rural Rehabilitation and Development with funding from a number of international donors including the World Bank, calls for each community to contribute ten percent toward the cost of each project (in either financial or labor contributions) (Isby 2010: 363). This allows for ordinary citizens to have a stake in each project. Citizen involvement also pressures local political authorities to become involved in the development and security of reconstruction projects. However, such local ownership assumes that that there is an incentive to not only initiate reconstruction projects, but also to maintain them over the long run.

\subsubsection{Strategies for Avoiding the Credible Commitment Trap}

Establishing the legitimacy of reconstruction officials and economic reforms is a crucial element of successful economic reconstruction. Yet finding solutions to the credible commitment trap is not simply a matter of establishing constraints on reformers. Those engaged in economic reconstruction efforts must simultaneously establish binding constraints on their courses of action and strongly signal to citizens and local political authorities that they are sincere in committing to their announced reforms. By doing so, repeated interactions between reconstruction officials and local citizens, as well as political authorities, can shift the equilibrium from a 'one shot' situation, where reformers may have the incentive to renege on their vocalized course of action or local citizens may not buy in to specific reforms, to an equilibrium of cooperation between citizens and those involved in leading reconstruction efforts. 
Filkins (2009) describes a discussion between General Stanley McChrystal, the former commander of U.S. troops and the International Security Assistance Force (ISAF) in Afghanistan, and Abdullah Jan, a governor in Garmsir in Afghanistan, which captures the essence of the credible commitment problem in economic reconstruction. Abdullah Jan told Gen. McChrystal the following:

Everyone in Garmsir sees that you are living in tents, and they know that you are going to be leaving soon. You need to build something permanent---a building. Because your job here is going to take years. Only then will people be persuaded that you are going to stay.

What Jan is expressing is the importance of reformers signaling a credible commitment to a future course of action. Absent such a signal, citizens will not see the future course of actions espoused by external reformers as legitimate.

Along with signaling credibility, reconstruction officials must seek to promote transparency and accountability, both within the Afghan and Iraqi national governments but also within their own operations. While such anti-corruption measures would be ideal if implemented in the first order, they clearly are not. This is likely to be due to the competing objectives of different reconstruction officials in the country. As a case in point, Mohammed Zia Salehi, an aide to the Karzai administration, was arrested on corruption charges but Karzai subsequently secured his release from prison. Later revelations indicated that Salehi was on the CIA's payroll. As described by Filkins and Mazzetti (2010), "Mr. Salehi's relationship with the CIA underscores deep contradictions at the heart of the Obama administration's policy in Afghanistan, with American officials simultaneously demanding that Mr. Karzai root out the corruption that pervades his government while sometimes subsidizing the very people suspected of perpetrating it." 
One strategy for overcoming credible commitment problems is for reconstruction officials to go overboard with reforms to signal the strength of their commitment to a stable policy regime (see Rodrik 1989). As an example where this strategy could be relevant, consider the case of Iraq, where private entrepreneurship is stifled by excessive regulations and corruption which raise the cost of opening a business (see Gunter, 2009). In order to signal a credible commitment to reform and support for private enterprise, reforms could simultaneously remove regulations on opening and closing a business while permanently closing the agencies that had previously enforced those regulations. This would send a credible signal to that current reforms are breaking from the past given that the previous means of enforcing those regulations are no longer available. However, reformers must be cognizant of how ongoing conflict influences perceptions regarding who the true reformers are and the legitimacy of announced reforms. For example, if citizens are not confident that property rights will be protected after conflict ends, removing barriers to business and investment will do little to generate development.

\subsection{The Knowledge Trap}

Knowledge plays a pivotal role in economic reconstruction efforts. As practitioners are wellaware, understanding the conditions at the 'grass-roots' level is essential for effective implementation of economic reforms. But acquiring, processing, and understanding this informal and indigenous knowledge is quite difficult, if not impossible. In the context of reconstruction, the knowledge trap results from the inability of reconstruction officials to have the information and knowledge necessary to craft context-specific reforms and policies. The assumption that external policy makers do have the requisite knowledge to design and implement first-best economic institutions in order to develop a market economy can be conceptualized as a 'fatal 
conceit'. Nobel Laureate F.A. Hayek (1988: 27) described the fatal conceit as the presumption that "man is able to shape the world around him according to his wishes." This is precisely the logic underpinning recent reconstruction efforts as illustrated by Stewart (2010), who notes that the plans for reconstruction in Afghanistan tap into "an Enlightenment faith that there is nothing intrinsically intractable about Afghan culture and society and that all men can be perfected (to a Western ideal) through the application of reason and the laws of social science."

To understand the importance of the knowledge trap, consider that a key part of most economic reconstruction efforts is the implementation of a market economy based on a multipronged plan. With such plans, it is implicitly assumed that (1) the knowledge necessary to effectively design a market economy can be possessed and (2) the consequences (negative or positive) of implementing this multi-pronged plan can be forecasted ex ante. However, attempts to plan and implement markets ignore the complex chain of experiments, choices, errors, and informal institutions which must emerge over time for functioning markets to operate. Robust markets are not planned, but are instead largely the result of emergent norms and institutions which facilitate interaction and exchange. The irony of the fatal conceit is the belief that planners have the sufficient information and knowledge to design and implement markets, which are desirable precisely because they are best able to deal with the fact that knowledge is dispersed and context specific (Hayek 1945).

This is especially important considering that many actors involved in reconstruction lack contextual knowledge that is crucial to accomplishing the desired ends. At the beginning of economic reconstruction efforts in Afghanistan, for instance, "governments, UN agencies, multinational lending institutions, universities, and NGOs were preparing concept papers on how to start reconstructing Afghanistan, but nobody had a clue about the country. None of the 
agencies had the capacity or the contracts to be able to consult Afghans about their basic needs or development priorities" (Rashid 2009: 171), Along with the classical knowledge pitfalls of economic planning, the lack of historical knowledge, cultural understanding, and general economic reconstruction experience in general significantly hampered efforts to rebuild the economies of Afghanistan and Iraq. ${ }^{51}$

\subsubsection{The Knowledge Problem}

The knowledge problem presents a significant hurdle for economic reconstruction efforts that take place during ongoing conflict. In general economic development practices, Pritchett and Woolcock (2004: 197), note that "valuable local 'practices'--idiosyncratic knowledge of variables crucial to the welfare of the poor (e.g. soil conditions, weather patterns, water flows)-get squeezed out, even lost completely, in large centralized development programs." Stephens and Ottaway (2005) provide evidence for this claim in the context of reconstruction efforts in Afghanistan, finding that the roof designs for schools and health clinics were not sufficient to support snowfall during the winter season, resulting in the collapse of many. Such experiences are not unexpected; Pritchett and Woolcock (2004) argue that neglecting local knowledge and engagement can lead to technological mistakes. The difficulties in Afghanistan and Iraq have been further exacerbated by the lack of security, which as discussed above threatens infrastructure projects even where they are successful.

Easterly (2006) argues that development efforts are led by two different groups of individuals: 'planners,' who engage in top-down administration and cannot acquire nor process dispersed and context-specific knowledge, and 'searchers,' who operate at the grass-roots level

\footnotetext{
${ }^{51}$ According to Isby (2010: 349), "the donors-international, governments, and NGOs—-have demonstrated too often a willingness to embrace quick-fix solutions and imported templates, with the context and true nature of Afghan society largely ignored.
} 
and utilize such knowledge to engage in effective economic and social development. In the context of economic reconstruction, one could refer to the above failures as an inevitable consequence of planners-led efforts whereas one could look at the successes of Greg Mortenson's construction of schools in Pakistan and Afghanistan, for instance, as a result of effectively utilizing the knowledge, connections, and historical experiences specific to particular villages and tribal elders (Mortenson and Relin 2006).

To illustrate the overemphasis on planning that pervades economic reconstruction efforts, consider again the U.S. military's counterinsurgency and stability operations field manuals, which inform military doctrine regarding economic reconstruction and provide a list of overarching institutional and macroeconomic goals. ${ }^{52}$ When economic reconstruction efforts take place during conflict, military officials are the central players given the need to secure peace. Therefore, it makes sense to consider the knowledge problem in the context of military led economic reconstruction.

Among the military's goals and objectives along 'logical lines of operations' for economic development are the (1) mobilization/development of local economic activity (manufacturing, services, agriculture); (2) initiation of contracts with local businesses to stimulate trade; (3) reconstruction of commercial infrastructure (banks, transportation, markets, currency); (4) support of broad-based economic opportunity (micro to macro development); (5) support of a free market economy (5-5). In the counterinsurgency field manual planning

\footnotetext{
${ }^{52}$ While the US Army/Marine Corps Counterinsurgency Field Manual and the Stability Operations Field Manual illustrate the U.S. military's overarching macroeconomic goals in economic reconstruction efforts, the manuals are first and foremost geared towards counterinsurgency operations.
} 
schematic, this multi-pronged plan is restoration of the "freedom to conduct lawful commerce" (U.S. Army/Marine Corps, 2007: 5-5). ${ }^{53}$

While positing strategies and prioritizing goals for economic reconstruction is necessary for developing the 'blueprint' for donor-led planning, the implementation of these plans is entirely different matter. These 'blueprints' for reconstruction and counterinsurgency efforts, while laudable in the abstract, capture the essence of the knowledge trap because they fundamentally assume that planners can design and implement a logical and cohesive plan to rebuild an entire economy. ${ }^{54}$

\subsubsection{Strategies for Avoiding the Knowledge Trap}

In order to carry out large-scale, top-down, and overarching macroeconomic reforms, exogenous reformers must necessarily abstract from complex context-specific intricacies such as local belief systems, context-specific knowledge of time and place, informal institutions, and historical experiences, because there is no feasible approach to accumulating this information in its entirety. Therefore, the only true solution to the knowledge trap is to recognize the binding knowledge constraints on human planning and take these lessons into account when contemplating or formulating future economic reconstruction efforts.

\footnotetext{
${ }^{53}$ Among the U.S. military's short-term goals for post-conflict recovery include the "(1) Security [of citizens] from insurgent intimidation and coercion, as well as from nonpolitical violence and crime; (2) Provision for basic economic needs; (3) Provision of essential services, such as water, electricity, sanitation, and medical care; (4) Sustainment of key social and cultural institutions; and (5) Other aspects that contribute to a society's basic quality of life" (U.S. Army/Marine Corps, 2007: 2-2). The military also seeks to engage in economic and infrastructure development (U.S. Army/Marine Corps, 2007: 1-19). These goals are relatively more tractable vis-à-vis the set of goals listed for overarching economic development. This is because (1) the knowledge required for such endeavors is not as context-specific, (2) they are immediate in time frame and are more readily implementable through standard military chains of logistics, and (3) do not involve the level of knowledge or logistics complexity required for overarching economic transformation.

${ }^{54}$ U.S. military planners are evidently self-aware of the shortcomings of this strategy. Indeed, in the counterinsurgency field manual, it is claimed that "many commanders are unfamiliar with the tools and resources required for promoting economic pluralism" (8-16). What is overlooked is whether military commanders and exogenous policymakers can ever possess the relevant knowledge to achieve the goals dictated by the counterinsurgency and stability operations field manuals. The knowledge problem - which emphasizes the importance of context-specific knowledge of time and place — suggests they cannot.
} 
Smaller scale reforms and actions, which make these knowledge constraints less binding (but not completely absent), are more likely to succeed (see Schilderman 2004). Decentralization of economic development efforts is especially important in Afghanistan. As noted by Rashid (2009: 197), "Afghanistan had always had a weak, decentralized state where the ruler governed through consensus over a confederation of tribes and ethnic groups." Administering aid projects through the auspices of the centralized Afghan national government is likely to be ineffective given the historical decentralized nature of Afghanistan's political decision making power (see Isby 2010). The demographics of the country also call for greater dispersion of policy decision making power. Afghanistan's population of roughly 30 million people is largely scattered among 20,000 remote, typically mountainous villages (Stewart, 2010).

There is some evidence that smaller scale movements can be effective. In the case of Iraq, for instance, Berman, Felter and Shapiro (2009) find that the violence-reducing effect of the $\$ 2.9$ billion in American reconstruction funds allocated through the Commander's Emergency Response Program (CERP) was found to be particularly effective after the troop surge. These funds were essentially allocated for small-scale projects and were at the discretion of military commanders. Because the surge led to an operational change in the way troops were deployedfor instance, greater forward operating base deployment and communication with indigenous actors - this led to a greater understanding of communities' needs and therefore a greater reduction in violence was seen after the change in tactics. CERP funds have also been administered in Afghanistan. Between fiscal years 2003 and 2009, CERP funds increased from $\$ 40$ million to $\$ 683$ million (Isby 2010: 358).

Of course, smaller-scale reforms and actions are less likely to influence the overarching framing of economic reconstruction efforts (Lyons 2009) yet a greater reliance on indigenous 
action may produce more effective results. Another strategy is to incorporate local citizens into the reconstruction design and process. By allowing those individuals with theknowledge (and incentives) specific to the contextual environment to lead reconstruction efforts, this can bypass the incapacity of planners to implement reforms and may also help to overcome the problems with domestic legitimacy and ownership associated with the credible commitment problem. In many cases, these individuals possess local knowledge of the language, culture, and history of the region and typically are seen as legitimate in the eyes of the citizens in these regions. Further,they understand the channels of logistics and have connections with fellow citizens. This can also mitigate aid 'blowback' where negative and violent unintended consequences may emerge after the initiation of reconstruction reforms.

The U.S. military has recognized the importance of local integration by the launching of Provincial Reconstruction Teams (PRTs) in Afghanistan. Allowing citizens, elders, and local government officials operating at the grass-roots to engage with individuals from NGOs, IGOs, and multinational corporations can lead to increased sharing of knowledge, ideas, beliefs, values, and feasible approaches to economic reconstruction. Yet problems have been noted with the increased employment of PRTs throughout the country. According to Isby (2010: 356), “...in more secure provinces, PRTs are sometimes duplicating Afghan government functions or competing with local officials. In November 2008, President Karzai complained that PRTs were creating a "parallel government" to his own in the countryside, and other Afghans perceived that many of the resources devoted to some PRTs were absorbed by multiple layers of contractors and sub-contractors, foreign and Afghan, so that little actually reached the grassroots."

In Afghanistan, a national development plan called the National Solidarity Program has proven to be highly successful (Rashid 2009: 184). The program allowed for committees in 
communities across the country to assess development priorities and were then allocated small grants (around \$30-60 thousand) for the implementation of development projects. As Rashid (2009: 185) illustrates, NGOs involved with the program "...helped villagers with the decision making process and in building projects such as digging wells and reservoirs and building bridges and schools." This decentralized approach to economic development is likely to be more successful given that it taps into the local knowledge of around 20,000 Community Development Councils (Zoellick 2008). As described by Miliband (2010), "[The village councils] have not just designed but implemented almost 40,000 development projects, and are now forming, from the bottom up, district councils." In addition to ameliorating the knowledge trap, this also aids in securing areas of the country because local citizens and political authorities have the incentive to maintain order and resist insurgents.

\subsection{The Political Economy Trap}

In conflict-stricken societies, economic reconstruction is one part of the broader strategy to eventually end conflict. However, economic reconstruction does not take place in isolation as economic and political reconstruction efforts are inextricably linked. For instance, if political institutions are characterized by corruption and unchecked power, this will stifle economic activity because property rights may be insufficiently protected or the regulatory hurdles (including bribes) to exchange, investment, and innovation may be too significant for local citizens or firms to overcome. Conversely, success (or failure) in different economic sectors may generate different political impulses by local citizens (e.g. support for less or more free trade,

levels of redistribution, etc.). The implementation of economic reforms within the context of political agreements is especially difficult (Del Castillo 2001). The political economy trap refers to the idealized view of politics and democracy which pervades reconstruction efforts. This view 
assumes that political and economic goals are compatible and neglects the tradeoff between democratic and economic outcomes. Further, this view overemphasizes the benefits of democratic political systems while understating the potential costs of such systems on economic reconstruction efforts.

\subsubsection{The Tradeoff Between Democracy and Economic Goals}

The political economy trap can adversely affect economic reconstruction in several key ways.

First, the idealized view of democracy overlooks the fact that unchecked democracy can produce a variety of negative outcomes. While reformers typically develop a comprehensive list of targets and goals to attain related to the establishment of democratic political systems in conflict torn countries (e.g. the holding of elections), implementation must be carefully performed. For instance, Flores and Nooruddin (2009: 5) find that "countries that undergo extensive democratization in the immediate postconflict period recover more slowly than countries that do not." This may be because "typically early elections in a highly polarized society empower elites, senior military leaders, and organized criminal elements" (U.S. Department of the Army, 2008: 1-18). This highlights how important the proper consolidation of democracy and institutions is for effective economic reconstruction, especially where conflict is still ongoing.

Absent effective constraints, democracy can produce illiberal outcomes-political, economic, and legal — that can do significant harm to efforts to foster economic reconstruction. When analyzing donor-led reconstruction goals in Afghanistan, Montgomery (2004: 36) illustrates some of the democracy-economic tradeoffs as such:

(1) the rule of law can degenerate into the rule of lawyers--litigious, costly, and dilatory; (2) economic efficiency can turn into profligacy--piratical and predatory; (3) free speech can reward superficiality and extremism; (4) the demand for unfulfilled rights can invite invidious reverse discrimination; (5) checked-andbalanced governmental institutions can yield policy stasis. 
The above five factors shed light on the need to establish effective constraints to ensure that reconstruction efforts result in stable political and economic orders. Yet creating these checks and balances is by no means a simple task, and social scientists and practitioners typically lack the knowledge of how to design effective comprehensive constitutional rules that will stick over the long run. This is because formal institutions, such as constitutions, must be grounded in informal customs and belief systems, which are largely beyond the reach of policy (North 2005; Boettke, Coyne and Leeson 2008). Where there is a disconnect between existing informal institutions and desired formal institutions, the latter will not be viewed as legitimate by citizens further contributing to the credible commitment trap discussed earlier.

Second, the idealized view of democracy often leads outsiders to overlook the tensions that may exist between the dual goals of establishing democratic political institutions and constructing the foundations of a market economy. These tensions may preclude the emergence of large-scale and first-best reforms; for instance, the privatization of state-owned enterprises or the removal of all tariffs and non-tariff barriers to trade may not occur because powerful interests drive policy efforts in the other direction. Allowing for self-determination through democracy can lead to demands by citizens that run counter to the free market economic plans often envisioned by reformers. When this happens, it presents a conundrum because implementing reconstruction plans requires either preventing democratic participation or ignoring the results.

For instance, when the highest ranking Shi'a Muslim scholar in Iraq, Grand Ayatollah al Sistani, issued a fatwa calling for general elections in June 2003, this led the CPA to ultimately abandon plans to appoint a body to construct a national constitution (Wong 2004). This had real effects on economic outcomes since the CPA was unable to have oversight over the writing of a constitution that could have provided a foundation for a permanent government, which would 
have led to stability and the encouragement of economic activity, which was viewed as a central aspect of the counter-insurgency strategy. Third, the overly optimistic view of democracy can lead to a neglect of the status quo. This happens because in assuming the ideal reformers often lose focus of the realities of the situation they face. Buchanan (1975) emphasizes that all reforms must start from the 'here and now' by recognizing the status quo and the associated constraints. The status quo refers to the array of existing formal and informal institutions, economic and political actors, and other particularities of post-conflict settings. Focus on the ideal, and the resulting failure to appreciate the status quo, has contributed to the failure of various reconstruction efforts. One well-known example can be found in Iraq where, instead of rebuilding governmental institutions from scratch, the CPA implemented a process of deBa'athification through the existing government apparatus. The intent of this policy was to minimize disruptions to the government's provision of services following the 2003, U.S.-led invasion. Yet as Allawi (2007: 161-2) explains, this proved problematic as the CPA did not have enough competent staff to operate the government. Hence, the lack of appreciation for the status quo-i.e. the recognition and understanding of pre-invasion governance structures and the associated binding constraints-has contributed to significant hurdles for economic reconstruction efforts in Iraq.

The status quo in both Afghanistan and Iraq also involved ongoing conflict, corruption, and power vacuums, which has made attempts to achieve both political and economic reconstruction that much more difficult. As in the case of the credible commitment problem, violent insurgencies in these countries have created uncertainty regarding the final distribution of political power. This political complexity has contributed to uncertainty regarding the dynamics of economic institutions and modes of coordination. Local citizens may be less likely to coalesce 
around economic reforms and reconstruction projects if their political demands lead them to join the ongoing insurgencies.

\subsubsection{Strategies for Avoiding the Political Economy Trap}

Those tasked with carrying out reconstruction efforts must be cognizant of the tradeoff between democratic goals and economic outcomes. The focus must be shifted away from the idealized view of democracy and towards finding ways to implement effective constraints on political decision makers to ensure that economic reconstruction efforts are not in conflict with political behaviors. However, given the uncertainty due to ongoing conflict, this can be difficult to accomplish. Recognizing, understanding, and appreciating the status quo and the associated constraints in post-conflict regions will provide a sounder foundation from which to implement policies.

Given the democracy-economy tradeoff, one possible strategy for overcoming the political economy trap is to prioritize political reconstruction over economic reconstruction or vice versa. For instance, del Castillo (2008: 41) argues that "should a conflict arise between peace (political) and development (economic) objectives, the first one should be paramount at all times. Because peace is a precondition for sustainable development, all actors should recognize and accept that political priorities will often constrain economic policymaking." According to General James Conway, Commandant of the Marine Corps, in fielding a question regarding the conditions that would be suitable for a significant troop withdrawal:

...a military force shapes the environment. We can't fix the economy, we can't fix the government. What we can do is affect the security. Now we can aid in these other things, but, for all intents and purposes, we shape the environment that allows these other lines of operations...to take place. Initially, in both Iraq now and in Afghanistan, we've had to do that at the start by ourselves. But increasingly, our doctrine calls for turning that over to host nation security forces...that's why we are partnering right now almost on every patrol with the 
Afghan security forces...that's why we want to posture the police so they can be successful...transitioning host nation forces to the point that they can do those things...when we think that we have sufficiently beaten down the insurgency in the area, we have sufficiently built up the Afghan capability to deal with what's there...then I think we have done the essence of what we were sent there to do (CSPAN 2010).

In some sense, the cessation of conflict and the promotion of security of person and property may very well be necessary and sufficient conditions to allow for economic growth and development. It should be realized that this approach places limits on the scale and scope of economic reconstruction efforts and implies that large-scale, first-best economic reforms are unlikely to be effective since they are more likely to conflict with political and security objectives. This can be problematic considering the historical and contemporary influences of conflict on human and social capital. This tension between political and economic goals is likely to be even greater in instances of ongoing conflict as efforts are focused on ending violence and securing peace given the uncertainty of the distribution of political power and nature and stability of political institutions.

\subsection{The Bureaucracy Trap}

There are appreciable limits to implementing economic reconstruction goals through bureaucratic channels. There have been scathing critiques of reconstruction efforts in Afghanistan and Iraq due to the lack of effective planning and cross-coordination among different agencies and reconstruction officials. There have also been numerous reports of corruption, waste, nepotism, and inefficiency, both on the part of the Afghan and Iraqi national governments but also by foreign reconstruction officials. For example, Stephens and Ottaway (2005) have documented the progress of a program to construct Afghan schools and health 
clinics, finding that the program suffered from a lack of coordination and poor planning due in part to the desire to have something completed before the 2004 Afghan presidential elections.

These inherent inefficiencies have led to continued calls for "improved coordination" and "better planning" on the part of bureaus. For instance, the U.S. Army's stability operations field manual (2008: 1-3) calls for 'unity of effort' among the array of actors involved in a stability operation, including the "synchronization, coordination, and/or integration of the activities of governmental and nongovernmental entities with military operations to achieve unity of effort." However, this focus on 'streamlining' bureaucracies places disproportionate emphasis on the role of bureaucracy in reconstruction efforts while neglecting the key role played by ordinary citizens acting in an entrepreneurial manner to solve the problems facing post-conflict societies. The bureaucracy trap emerges when there is an overreliance on bureaucracies in economic reconstruction efforts. Avoiding this trap therefore involves an understanding of the industrial organization of bureaucracies, including the incentives and constraints faced by bureaucrats, as well as an appreciation for the capacity for 'searchers' to coordinate scarce resources and find solutions to problems. ${ }^{55}$

\subsubsection{Bureaucracy and Economic Reconstruction}

Agencies engaged in economic reconstruction efforts receive their budgets from an array of government actors. Budgets are allocated based on relationships with these actors, as well as on the logistical and compensatory needs of each bureau. Yet financial resources are scarce and each bureau is therefore competing with other agencies over a limited budget. The incentives created by this process result in predictable behaviors among those working in these

\footnotetext{
55 There is an array of bureaucracies involved in post-conflict economic reconstruction including government agencies, IGOs and NGOs. Although our analysis could be applied to any of these organizations, we limit our focus to government agencies since they are typically the largest bureaucracies involved in economic reconstruction and usually play the largest role in terms of planning, implementation and oversight of reconstruction plans.
} 
bureaucracies and these behaviors influence the process of economic reconstruction in a number of ways.

First, bureaus involved in economic reconstruction efforts will attempt to receive the largest possible share of financial resources and the associated influence over reconstruction efforts. This typically involves investing resources in signaling the relative importance of one bureau over others. Second, this intrinsically creates an important tension in reconstruction efforts: agencies should be united in the common goals of reconstruction (e.g. security, amelioration of poverty, infrastructural development, etc.) yet they are instead competing with one another for funds and influence. This often leads to efforts to develop a specialization that differentiates one agency from the others in order to receive a larger part of the fixed budget. Each bureau has its own agenda, which may clash with the agendas of other agencies as well as with the overarching goal of successful economic reconstruction. Third, bureaus will tend to exhaust their entire budgets while continually seeking financial appropriations in order to increase the size and scope of the agency. The failure of a bureau to spend its allocated budget typically leads to budget reductions in subsequent years. This creates the incentive to ensure that budgets are exhausted even if wasteful expenditures are necessary to achieve this outcome. This inherently runs counter to ensuring that reconstruction costs are minimized while the benefits to the citizens are maximized. This waste is magnified by the lack of adequate feedback mechanisms in bureaucratic settings making it difficult for bureaucrats to accurately gauge the effective allocation, and reallocation, of resources to high-return uses (Mises 1983). Lastly, bureaucracies are typically judged on the basis of readily observable outputs which do not 
necessarily coincide with the goals of long-run economic reconstruction and development (see Easterly 2003). ${ }^{56}$

These four factors have had substantial impacts on reconstruction efforts in Iraq and Afghanistan. For instance, infighting for control over Iraq reconstruction policy between the U.S. Department of Defense and the U.S. State Department became highly acrimonious (Phillips 2005: 7). Diamond (2005: 28-9) claims that "a number of U.S. government agencies had a variety of visions of how political authority would be reestablished in Iraq. In the bitter, relentless infighting among U.S. government agencies in advance of the war, none of these preferences clearly prevailed.”

Yet another factor affecting the capacity for bureaucracies to effectively engage in economic reconstruction is decision making and the flow of information within these organizational structures. Bureaucratic rules tend to be rigid in nature which often prevents flexibility to rapidly changing conditions. This poses a problem for economic reconstruction efforts which typically take place in the context of uncertainty and constant change-especially in the cases of Afghanistan and Iraq, where violent insurgencies are still ongoing. As Rondinelli (2004: 12) explains, "Many of the decisions about how to promote the development of Afghanistan are likely to be made rapidly, reactively, and in response to uncertain and everchanging political forces. Careful deliberation is likely to be in short supply in the face of rapidly changing political trends and complex social and cultural conditions in Afghanistan."

To provide a concrete example of this logic, consider the economic reconstruction of Iraq, where bureaucratic waste and inefficiency have been magnified as a result of the absence of

\footnotetext{
56 According to Rashid (2009: 177), at the beginning of economic reconstruction efforts in Afghanistan, "Quick impact projects became a Washington favorite. These were swift and cheap, such as digging a well, rebuilding a small bridge, or repairing a broken-down school building, and were supposed to convince the population that reconstruction was moving ahead. Instead, such projects invariably helped only the local warlord or commander the CIA was supporting."
} 
effective feedback and accountability mechanisms (Glanz 2006). Specifically, consider the $\$ 644$ million "Community Stabilization Program" (CSP) in Iraq, which was suspended due to significant fraud and waste. An audit by the USAID's Inspector General found that some of the funds allocated specifically for weakening the insurgency actually went to insurgents, as well as to corrupt community leaders and CSP representatives (USAID Office of the Inspector General, 2008: 8). As another example, consider that a more recent audit published in July 2010 found that that U.S. Department of Defense was unable to property account for $96 \%$ of the $\$ 9.1$ billion funds it has received since 2004 through the Development Fund for Iraq for reconstruction activities (Special Inspector General for Iraq Reconstruction 2010).

In Afghanistan, the story has not been much different. The United States spent around \$860 million on police training, with Rashid (2009: 205) concluding that the "results were almost totally useless." This is because "DynCorp [a State Department contractor] was training the police to fight an insurgency rather than win hearts and minds in their localities." The actions of the local police thereafter were observed to be as "rapacious" as they were before (Rashid 2009: 205). According to the former finance minister of Afghanistan, Ashraf Ghani, "International technical assistance is considered to be largely wasted...hundreds of millions of dollars have gone into technical assistance only to increase corruption and misgovernance" (Isby 2010: 334).

\subsubsection{Strategies for Avoiding the Bureaucracy Trap}

The overemphasis on improving coordination and planning within and between bureaus ignores the constraints on what bureaucracies can actually achieve in economic reconstruction during conflict. Coordination is a necessary but insufficient condition for successful economic reconstruction because real constraints exist on bureaucratic activity no matter what the level of 
coordination. Perhaps more importantly, this focus puts unrealistic faith in increased bureaucratic central planning, which is the very antithesis of the free markets that reconstruction authorities seek to engender through economic reconstruction.

The bureaucracy trap illustrates that overreliance on bureaus to coordinate reconstruction efforts can hamper economic growth and development. The main concern is that the central planning associated with bureaucracy results in the systematic disregard for the capacity of ordinary citizens and 'searchers' to act entrepreneurially to find solutions for the problems of economic reconstruction. One example of the power of private initiative is the mobile phone industry in Iraq (The Economist, 2009). With 20 million subscribers in a county of 27 million citizens, mobile phones have become a tool of commerce and have allowed for greater interpersonal communication. According to Isby (2010: 343), in Afghanistan "communications and the spread of cell phones have proven a great success, funded almost exclusively by the private sector, and has managed to avoid corruption. This was largely carried out by Afghans working in the private sector, borrowing money and acquiring technology from outside as required, rather than relying on donor nations." While many reconstruction activities must inherently be dealt with through bureaucratic channels, it is critical to realize the limits of bureaucratic activity and the importance of private initiative and decentralization for sustainable economic recovery.

\subsection{Conclusion}

The overarching implication of our analysis is that there are significant constraints on what economic reconstruction can accomplish. These constraints are even more significant in cases of ongoing conflict and insurgency since this context contributes to additional tensions between political and military actors, as well as citizens. Reconstruction amidst violence places the 
military at the core of the reconstruction effort and leads to a tension in various goals and strategies which often leads to outcomes which run counter to the goals of economic reconstruction. Moreover, the involvement of the military in economic reconstruction stretches military personnel beyond their comparative advantage - the use of force - and asks them to act on knowledge and information which they cannot possibly possess.

The economic reconstruction traps put forth in this paper indicate that the first-best, and often the second- and third-best policies, will not be realistic given the incentives, constraints, and knowledge limitations inherent to economic reconstruction efforts. This implication may be apparent, but as the many examples discussed in this paper indicate, actual efforts to engage in economic reconstruction amidst conflict tend to neglect these traps and the resulting constraints. The failure to appreciate the traps of economic reconstruction sheds light on many of the failures in Afghanistan and Iraq to date and provide insight into what should be avoided in future efforts in similar settings.

Finally, we must point out that another possibility not captured in our analysis is that the failure of economic reconstruction may not be due specifically to the traps discussed above, but rather because of inappropriate or irrelevant strategies and policies underpinning the initial occupation and reconstruction. Our analysis indicates that even under a first-best scenario, where the initial occupation and reconstruction is legitimate and based on sound strategies and policies, the desired end of economic reconstruction may not be achieved due to some combination of the four reconstruction traps. If we ease this assumption and recognize that inappropriate policies and strategies may underpin the initial occupation and reconstruction, this provides even more reason to be skeptical that economic reconstruction officials will be successful in achieving their goals during protracted insurgencies. 


\section{Bibliography}

Acemoglu, Daron, Simon Johnson, and James A. Robinson. 2001. "The Colonial Origins of Comparative Development: An Empirical Investigation." American Economic Review, 91(5): 1369-1401.

Acemoglu, Daron, and Simon Johnson. 2005. "Unbundling Institutions." Journal of Political Economy, 113(5): 949-995.

Acemoglu, Daron and James A. Robinson. 2006. Economic Origins of Dictatorship and Democracy. Cambridge, UK: Cambridge University Press.

Adam, Christopher, Paul Collier, and Victor Davies. 2008. "Post-conflict Monetary Reconstruction." World Bank Economic Review, 22(1), 87-112.

Addison, Tony and Tilman Brück (eds.) 2009. Making Peace Work: The Challenges of Social and Economic Reconstruction. New York, NY: Palgrave-Macmillian.

Akhmedov, Akhmed, and Ekaterina Zhuravskaya. 2004. "Opportunistic Political Cycles: Test in a Young Democracy Setting." Quarterly Journal of Economics, 119(4): 1301-1338.

Akresh, Richard, Philip Verwimp, and Tom Bundervoet. 2011. "Civil War, Crop Failure, and Child Stunting in Rwanda." Economic Development and Cultural Change, 59(4): 777810.

Akresh, Richard, Sonia Bhalotra, Marinella Leone, and Una Osili. 2011. "War and Stature: Growing Up During the Nigerian Civil War." American Economic Review (Papers \& Proceedings), forthcoming.

Akresh, Richard and Damien De Walque. 2010. "Armed Conflict and Schooling : Evidence from the 1994 Rwandan Genocide." Working paper. Available at http://ideas.repec.org/p/hic/wpaper/47.html

Allawi, Ali A. 2007. The Occupation of Iraq: Winning the War, Losing the Peace. New Haven: Yale University Press.

Autesserre, Séverine. 2010. The Trouble With the Congo: Local Violence and the Failure of International Peacebuilding. Cambridge: Cambridge University Press.

Baird, Sarah, Jed Friedman, and Norbert Schady. 2011. "Aggregate Income Shocks and Infant Mortality in the Developing World.” Forthcoming, Review of Economics and Statistics.

Bastian, Sunil and Robin Luckham (eds.) 2003. Can Democracy Be Designed? The Politics of Institutional Choice in Conflict-Torn Societies. New York: Zed Books. 
Baum, Christopher F., Mark E. Schaffer, and Steven Stillman. "Ivreg2: Stata Module for Extended Instrumental Variables/2SLS, GMM and AC/HAC, LIML and K-Class Regression." Available at http://ideas.repec.org/c/boc/bocode/s425401.html.

Bellows, John and Edward Miguel. 2006. "War and Institutions: New Evidence from Sierra Leone." American Economic Review, 96(2): 394-399.

Bellows, John and Edward Miguel. 2009. "War and Collective Action in Sierra Leone." Journal of Public Economics, 93(11-12): 1144-1157.

Berman, Eli, Jacob N. Shapiro, and Joseph H. Felter. 2009. "Can Hearts and Minds be Bought? The Economics of Counterinsurgency in Iraq." NBER Working Paper, 14606.

Besley, Timothy and Torsten Persson. 2011. Pillars of Prosperity: The Political Economics of Development Clusters. Princeton University Press, New Jersey.

Blattman, Christopher. 2009. "From Violence to Voting: War and Political Participation in Uganda." American Political Science Review, 103(2): 231-247.

Blattman, Christopher and Edward Miguel. 2010. "Civil War." Journal of Economic Literature, 48(1): 3-57.

Boettke, Peter, Christopher J. Coyne, and Peter T. Leeson. 2008. "Institutional stickiness and the new development economics." American Journal of Economics and Sociology, 67(2), 331-358.

Bohn, Frank, and Pierre-Guillaume Meon. 2009. "Political Transfer Cycles." NiCE Working Paper 09-101.

Boyce, James K. (ed.). 1996. Economic Policy for Building Peace: The Lessons of El Salvador. Boulder, CO: Lynne Reinner.

Boyce, James K. 2002. Investing in Peace: Aid and Conditionality after Civil Wars. Oxford: Oxford University Press.

Brakman, Steven, Harry Garretsen, and Marc Schramm. 2004. "The Strategic Bombing of Cities in Germany in World War II and Its Impact on City Growth." Journal of Economic Geography, 4(1): 1-18.

Brender, Adi, and Allan Drazen. 2005. "Political Budget Cycles in New Versus Established Democracies." Journal of Monetary Economics, 52(7): 1271-1295.

Brender, Adi, and Allan Drazen. 2008. "How Do Budget Deficits and Economic Growth Affect Reelection Prospects? Evidence from a Large Panel of Countries." American Economic Review, 98(5): 2203-2220. 
Buchanan, James M. 1975. The Limits of Liberty: Between Anarchy and Leviathan. Chicago: University of Chicago Press.

Bundervoet, Tom, Philip Verwimp, and Richard Akresh. 2009. "Health and Civil War in Rural Burundi." Journal of Human Resources, 44(2): 536-563.

Chamarbagwala, Rubiana and Hilcías E. Morán. 2011. "The Human Capital Consequences of Civil War: Evidence from Guatemala." Journal of Development Economics, 94(1): 4161.

Chaudhury, Nazmul, Jeffrey Hammer, Michael Kremer, Karthik Muralidharan and F. Halsey Rogers. 2006. "Missing in Action: Teacher and Health Worker Absence in Developing Countries." Journal of Economic Perspectives, 20(1): 91-116.

Chauffour, Jean-Pierre. 2011. "On the Relevance of Freedom and Entitlement in Development: New Empirical Evidence (1975-2007).” World Bank Policy Research Working Paper No. 5660 .

Chimni, B.S. (2003) "Post-conflict peace-building and the return of refugees: concepts, practices, and institutions," In, Refugees and Forced Displacement: International Security, Human Vulnerability, and the State, Edward Newman and Joanne van Selm (eds.), New York: United Nations University Press.

Coalition Provisional Authority (CPA) (2003) CPA official website: http://www.cpairaq.org/regulations/. Last Accessed December 19, 2009.

Cole, Shawn. 2009. "Fixing Market Failures or Fixing Elections? Agricultural Credit in India." American Economic Journal: Applied Economics 1(1): 219-250.

Collier, Paul. 1994 "Demobilization and Insecurity: A Study in the Economics of the Transition from War to Peace." Journal of International Development, 6(3), 343-351.

Collier, Paul. 2009. "Post-conflict Recovery: How Should Policies be Distinctive?" Journal of African Economics, 18(suppl 1): i99-i131.

Collier, Paul, V.L. Elliot, Håvard Hegre, Anke Hoeffler, Marta Reynal-Querol, and Nicholas Sambanis. 2003. Breaking the Conflict Trap: Civil War and Development Policy, Washington, D.C.: The World Bank.

Collier, Paul and Anke Hoeffler. 2000. "Aid, Policy, and Growth in Post-Conflict Societies." European Economic Review, 48(4): 1125-1145.

Council on Foreign Relations. 2005. "In the Wake of War: Improving US Post-Conflict Capabilities." Available at http://www.cfr.org 
Coyne, Christopher J. 2008. After War: The Political Economy of Exporting Democracy. Stanford Economics and Finance.

Coyne, Christopher J. and Peter Boettke. 2009. "The Problem of Credible Commitment in Reconstruction.” Journal of Institutional Economics, 5(1), 1-23.

Coyne, Christopher J. and Adam Pellillo. 2011. "The Art of Seeing Like a State: State-building in Afghanistan, the DR Congo, and Beyond." Review of Austrian Economics, forthcoming.

CSPAN. 2010. "War in Afghanistan and Marine Corps Policies." Transcript and video available at http://www.c-spanvideo.org/program/295155-1

Cutler, David, Angus Deaton, and Adriana Lleras-Muney. 2006. "The Determinants of Mortality." Journal of Economic Perspectives, 20(3): 97-120.

Davis, Donald R. and David E. Weinstein. 2002. "Bones, Bombs, and Break Points: The Geography of Economic Activity." American Economic Review, 92(5): 1269-1289.

Del Castillo, Graciana. 2001. "Post-conflict Reconstruction and the Challenge to International Organizations: The Case of El Salvador.” World Development, 29(12), 1967-1985.

Del Castillo, Graciana. 2008. Rebuilding War-Torn States: The Challenges of Post-Conflict Economic Reconstruction. New York: Oxford University Press.

Demekas, Dimitri G., James McHugh, and Theodora Kosma. 2002. "The Economics of Post Conflict Aid." IMF Working Paper WP/02/198. Available at http://www.imf.org/external/pubs/ft/wp/2002/wp02198.pdf.

Desai, Raj M. and Homi Kharas. 2010. “The Determinants of Aid Volatility.” Brookings, Global Economy \& Development Working Paper No. 42.

Diamond, Larry. 2005. Squandered Victory: The American Occupation and the Bungled Effort to Bring Democracy to Iraq. New York: Henry Holt.

Do, Quy-Toan and Lakshmi Iyer. 2010. "Geography, Poverty and Conflict in Nepal.” Journal of Peace Research, 47(6): 735-748.

Dobbins, James, John G. McGinn, Keith Crane, Seth G. Jones, Rollie Lal, Andrew Rathmell, Rachel M. Swanger, and Anga R. Timilsina. 2003. America's Role in Nation-Building: From Germany to Iraq. Santa Monica, CA: RAND Corporation.

Dobbins, James, John G. McGinn, Keith Crane, Seth G. Jones, Rollie Lal, Andrew Rathmell, Rachel M. Swanger, and Anga R. Timilsina. 2005. The U.N. 's Role in Nation-Building: From the Congo to Iraq. Santa Monica, CA: RAND Corporation. 
Drazen, Allan. 2001. "The Political Business Cycle After 25 Years." In Ben S. Bernanke and Kenneth Rogoff (eds.), NBER Macroeconomics Annual 2000, Volume 15. MIT Press.

Drazen, Allan, and Marcela Eslava. 2010. "Electoral manipulation via voter-friendly spending: Theory and evidence.” Journal of Development Economics 92(1): 39-52.

Easterly, William. 2003. "The Cartel of Good Intentions: The Problem of Bureaucracy in Foreign Aid." Journal of Policy Reform, 5(4), 223-250.

Easterly, William. 2006. The White Man's Burden. New York: The Penguin Press.

Eck, Kristine. 2012. "In Data We Trust? A Comparison of UCDP GED and ACLED Conflict Events Datasets." Cooperation and Conflict, forthcoming.

Fathi, Nazila. 2009. "Ex-Leader of Iran's Revolutionary Guard Seeks Presidency." The New York Times, April 22, 2009.

Fearon, James and Davis Laitin. 2003. "Ethnicity, Insurgency, and Civil War." American Political Science Review, 97(1): 75-90.

Fearon, James. 2007. "Iraq's civil war.” Foreign Affairs, 86(2), 2-15.

Filkins, Dexter. 2009. “Stanley McChrystal's Long War.” The New York Times, October 14.

Filmer, Deon and Lant H. Pritchett. 2001. "Estimating Wealth Effects without Expenditure Dataor Tears: An Application to Educational Enrollments in States of India." Demography, 38(1): 115-132.

Flores, Thomas Edward and Irfan Nooruddin. 2009. "Democracy Under the Gun: Understanding Postconflict Recovery." Journal of Conflict Resolution, 53(1), 3-29.

Franzese, Robert J. 2002. "Electoral and Partisan Cycles in Economic Policies and Outcomes." Annual Review of Political Science, 5(1): 369-421.

Garrett, Nicholas and Laura Seay. 2011. "Trade, Development, and Peacebuilding in the African Great Lakes: The Role of the Minerals Sector." In Ramsbotham, Alexander and I William Zartman (eds.), Paix Sans Frontières: Building Peace Across Borders. Accord: An International Review of Peace Initiatives, Issue 22.

Garrett, Thomas A. and Russell S. Sobel. 2003. "The Political Economy of FEMA Disaster Payments." Economic Inquiry, 41(3): 496-509.

Ghosh, Arkadipta. 2006. "Electoral Cycles in Crime in a Developing Country: Evidence from the Indian States." Working paper. 
Glaeser, Edward L., Rafael La Porta, Florencio Lopez-de-Silanes, and Andrei Shleifer. 2004. "Do Institutions Cause Growth?” Journal of Economic Growth, 9: 271-303.

Glanz, James. 2006. "Audit Describes Misuse of Funds in Iraq Projects.” The New York Times, January 25.

Global Witness. 2009. War and the Militarisation of Mining in Eastern Congo. Global Witness Report.

Grier, Robin and Kevin Grier. 2000. "Political Cycles in Nontraditional Settings: Theory and Evidence from the Case of Mexico." Journal of Law and Economics, 43(1): 239-263.

Grier, Kevin. (2008). "US Presidential Elections and Real GDP Growth, 1961-2004." Public Choice, 135(3): 337-352.

Gunter, Frank R. 2009. “Liberate Iraq's economy.” The New York Times, November 15.

Hafner-Burton, Emilie M., Susan D. Hyde, and Ryan S. Jablonski. 2010. "Terrorizing Freedom: When Governments Use Repression to Manipulate Elections.” Working Paper.

Haggard, Stephan and Lydia Tiede. 2011. "The Rule of Law and Economic Growth: Where Are We?" World Development, forthcoming.

Hayek, F.A. 1945. "The Use of Knowledge in Society.” American Economic Review, 35(4), 519530.

Hayek, F.A. 1988. The Fatal Conceit: The Errors of Socialism. Chicago: Chicago University Press.

Hegre, Håvard, Gudrun Østby, and Clionadh Raleigh. 2009. "Poverty and Civil War Events: A Disaggregated Study of Liberia.” Journal of Conflict Resolution, 53(4): 598-623.

Ikenberry, G. John. 2001. After Victory: Institutions, Strategic Restraint, and the Rebuilding of Order after Major Wars. Princeton: Princeton University Press.

International Rescue Committee (IRC). 2004. Coghlan, Brennan et al. Mortality in the Democratic Republic of the Congo: Results from a Nationwide Survey Conducted AprilJuly 2004. Melbourne and New York: Burnet Institute, International Rescue Committee, 2004: Volume 26.

Isby, David. 2010. Afghanistan: Graveyard of Empires - A New History of the Borderland. New York: Pegasus Books.

Kinney, Mary et al. 2010. "Sub-Saharan Africa's Mothers, Newborns, and Children: Where and Why Do They Die?" PLoS Medicine 7(6): e1000294. 
Kondylis, Florence. 2010. "Conflict Displacement and Labor Market Outcomes in Post-War Bosnia and Herzegovina." Journal of Development Economics, 93(2): 235-248.

Kudamatsu, Masayuki. 2011. "Has Democratization Reduced Infant Mortality in Sub-Saharan Africa? Evidence from Micro Data." Forthcoming, The Journal of the European Economic Association.

Kudamatsu, Masayuki, Torsten Persson, and David Strömberg. 2011. "Weather and Infant Mortality in Africa." Working paper.

Kumar, Krishna (ed.). 1998. Postconflict Elections, Democratization, and International Assistance. Boulder: Rienner.

Kyle, Jordan. 2010. "The Political Spoils of War: The Impact of Conflict on Political Participation in Aceh, Indonesia." Working paper. Available at http://www.columbia.edu/cu/polisci/pdf-files/apsa_kyle.pdf.

Laakso, Liisa. 2002. "The Politics of International Election Observation: The Case of Zimbabwe in 2000." The Journal of Modern African Studies 40(3): 437-464.

Lawn, Joy E et al. 2009. "Two Million Intrapartum-Related Stillbirths and Neonatal Deaths: Where, Why, and What Can be Done?" International Journal of Gynecology and Obstetrics, 107 Suppl : S5-18, S19.

Lyons, Michal. 2009. "Building Back Better: The Large-Scale Impact of Small-Scale Approaches to Reconstruction." World Development, 37(2): 385-398.

McChrystal, Stanley. 2009. COMISAF Initial Assessment. Available at http://www.washingtonpost.com/wpdyn/content/article/2009/09/21/AR2009092100110.h tml

MEASURE DHS, ICF International. 2012. "Description of the Demographic and Health Surveys - Individual Recode Data File.” MEASURE DHS (DHS V) Version 1.0. March 2, 2012. http://www.measuredhs.com/pubs/pdf/DHSG4/Recode5DHS_2March2012.pdf

Melander, Erik and Ralph Sundberg. 2011. "Climate Change, Environmental Stress, and Violent Conflict-Test Introducing the UCDP Georeferenced Event Dataset." Paper presented at the International Studies Association, March 16-19, Montreal, Canada.

Miguel, Edward and Gerard Roland. 2011. "The Long Run Impact of Bombing in Vietnam." Journal of Development Economics, 96(1): 1-15.

Miliband, David. 2010. "How to End the War in Afghanistan." New York Review of Books, April 29, 2010. 
Miller, Grant, and B.P. Urdinola. 2010. "Cyclicality, Mortality, and the Value of Time: The Case of Coffee Price Fluctuations and Child Survival in Colombia." Journal of Political Economy 118(1): 113-155.

Minoiu, Camelia and Olga N. Shemyakina. 2012. "Armed Conflict, Household Victimization, and Child Health in Côte d'Ivoire.” ECINEQ Working Paper 2012-245.

Mises, Ludwig. 1935 [1920]. "Economic Calculation in the Socialist Commonwealth," in F.A. Hayek (ed.) Collectivist Economic Planning. London: Routledge \& Kegan Paul.

Mises, Ludwig. (1983) Bureaucracy. Grove City, PA: Libertarian Press, Inc

Mohammed, Riyadh and John Leland. 2009. "In Heart of Iraq, a Plan to Revive the Pulse of a Central Artery." The New York Times, December 29.

Montgomery, John D. and Dennis A. Rondinelli. (eds.). 2004. Beyond Reconstruction in Afghanistan: Lessons from Development Experience. New York: Palgrave MacMillan.

Montgomery, John D. 2004. "Supporting postwar aspirations in Islamic societies." In Montgomery, J.D. and Rondinelli, D.A. (eds.). Beyond Reconstruction in Afghanistan: Lessons from Development Experience. New York: Palgrave MacMillan.

Mortenson, Greg and David Oliver Relin. 2006. Three Cups of Tea: One Man's Mission to Promote Peace... One School at a Time. New York: Penguin Books.

Nest, Michael, Francois Grignon, and Emizet F. Kisangani. 2006. The Democratic Republic of the Congo: Economic Dimensions of War and Peace. International Peace Academy Occasional Paper Series. Boulder: Lynne Rienner Publishers.

Norland, Rod. 2010. "Poison Gas Used on Girls' Schools, Afghan Officials Say." The New York Times, August 31.

North, Douglass. 2005. Understanding the Process of Economic Change. New Jersey: Princeton University Press.

Nzongola-Ntalaja, Georges. 2002. The Congo: From Leopold to Kabila. New York: Zed Books.

Office of the United Nations High Commissioner for Human Rights (OHCHR). 2010. “'Report of the Mapping Exercise Documenting the Most Serious Violations of Human Rights and International Humanitarian Law Committed within the Territory of the Democratic Republic of the Congo between March 1993 and June 2003." Available, with comments by

states, at http://www.ohchr.org/EN/Countries/AfricaRegion/Pages/RDCProjetMapping.aspx . 
Pepinsky, Thomas. 2007. "Autocracy, Elections, and Fiscal Policy: Evidence from Malaysia." Studies in Comparative International Development 42(1-2): 136-163.

Persson, Torsten and Guido Tabellini. 2005. The Economic Effects of Constitutions. MIT Press.

Phillips, David L. 2005. Losing Iraq: Inside the Postwar Reconstruction Fiasco. New York: Basic Books.

Potrafke, Niklas. 2010. "The growth of public health expenditures in OECD countries: do government ideology and electoral motives matter?" Journal of Health Economics 29(6): 797-810.

Pritchett, Lant and Michael Woolcock. 2004. "Solutions when the Solution is the Problem: Arraying the Disarray in Development." World Development, 32(2): 191-212.

Prunier, Gerard. 2009. Africa's World War: Congo, the Rwandan Genocide, and the Making of a Continental Catastrophe. Oxford: Oxford University Press.

Raleigh, Clionadh, Andrew Linke, Håvard Hegre, and Joakim Karlsen. 2010. "Introducing ACLED-Armed Conflict Location and Event Data." Journal of Peace Research, 47(5): $1-10$.

Rashid, Ahmed. (2009) Descent into Chaos: The United States and the Failure of Nation Building in Pakistan, Afghanistan, and Central Asia. New York: Penguin Books.

Rathmell, Andrew. 2005. "Planning Post-Conflict Reconstruction in Iraq: What Can We Learn?" International Affairs, 81(5): 1013-1038.

Rodrik, Dani. 1989. "Promises, Promises: Credible Policy Reform Via Signaling." The Economic Journal, 99(397): 756-772.

Rodrik, Dani, Arvind Subramanian, and Francesco Trebbi. 2004. "Institutions Rule: The Primacy of Institutions over Geography and Integration in Economic Development." Journal of Economic Growth, 9: 131-165.

Rogoff, Kenneth, and Anne Sibert. 1988. "Elections and Macroeconomic Policy Cycles." Review of Economic Studies 55(1): 1-16.

Rondinelli, Dennis A. 2004. "International goals and strategies for Afghanistan's development: reconstruction and beyond." In Montgomery, J.D. and Rondinelli, D.A. (eds.) Beyond Reconstruction in Afghanistan: Lessons from Development Experience. New York: Palgrave Macmillan.

Rotberg, Robert I. 2004. "The Failure and Collapse of Nation-States: Breakdown, Prevention, and Repair." In Rotberg (ed.), When States Fail: Causes and Consequences. Princeton: Princeton University Press. 
Rutstein, Shea Oscar and Guillermo Rojas. 2006. "Guide to DHS Statistics.” Demographic and Health Surveys, ORC Macro, Calverton, MD. September 2006.

Schady, Norbert R. 2000. "The Political Economy of Expenditures by the Peruvian Social Fund (FONCODES), 1991-95.” American Political Science Review 94(2): 289-304.

Schilderman, T. (2004) "Adapting traditional shelters for disaster mitigation and reconstruction: experiences with community-based approaches." Building Research and Information, 32(5), 414-426.

Schuknecht, Ludger. 1996. "Political Business Cycles and Fiscal Policies in Developing Countries.” Kyklos, 49(2): 155-170.

Shemyakina, Olga. 2011. "The Effect of Armed Conflict on Accumulation of Schooling: Results from Tajikistan.” Journal of Development Economics, 95(2): 186-200.

Shi, Min, and Jakob Svensson. 2006. "Political budget cycles: Do they differ across countries and why?" Journal of Public Economics 90(8-9): 1367-1389.

Smith, Adam. 1776 [1976]. An Inquiry into the Nature and the Causes of the Wealth of Nations. Chicago: University of Chicago Press.

Sobel, Russell S. and Adam Pellillo. 2011. "The Politics of Elections and Congressional Oversight." Forthcoming, Michael Reksulak, Laura Razzolini, and William F. Shughart, II (Eds.), The Elgar Companion to Public Choice, $2^{\text {nd }}$ Edition.

Sobhan, Rehman. 2002. "Aid Effectiveness and Policy Ownership." Development and Change, 33(3), 539-548.

Special Inspector General for Iraq Reconstruction (2010) "Development fund for Iraq: Department of Defense needs to improve financial and management controls." July 27, SIGIR 10-020.

Stearns, Jason. 2011. Dancing in the Glory of Monsters: The Collapse of the Congo and the Great War of Africa. New York: PublicAffairs.

Stephens, Joe and David B. Ottaway. 2005. "A Rebuilding Plan Full of Cracks." The Washington Post November 20.

Stewart, Rory. 2010. “Afghanistan: What Could Work.” The New York Review of Books, 57(1), January 14, 2010 Issue.

Sundberg, Ralph, Mathilda Lindgren, and Ausra Padskocimaite. 2010. "UCDP GED Codebook Version 1.0-2011.” Department of Peace and Conflict Research, Uppsala University. 
The Economist. 2009. "Iraq's mobile-phone revolution: better than freedom?" November 14.

Transparency International. (2009) Corruption perceptions index 2009. Accessed online at http://www.transparency.org/policy_research/surveys_indices/cpi/2009/cpi_2009_table on January 10, 2010.

Tufte, Edward R. 1978. Political Control of the Economy. Princeton, NJ: Princeton University Press.

Turner, Thomas. 2007. The Congo Wars: Conflict, Myth, and Reality. New York: Zed Books.

United States Agency for International Aid, Office of the Inspector General. 2008. "Audit of USAID/Iraq's community stabilization program." Audit Report No. E-267-08-001-P. Available online at http://pdf.usaid.gov/pdf_docs/PDACN242.pdf.

U.S. Army/Marine Corps (2007) Counterinsurgency Field Manual (U.S. Army Field Manual No. 3-24/Marine Corps Warfighting Publication No. 3-33.5 Chicago: University of Chicago Press.

U.S. Department of the Army (2008) Stability Operations Field Manual (U.S. Army Field Manual No. 3-07), University of Michigan Press.

Williams, Garland H. 2003. "Post Conflict Reconstruction: On the Critical Path to Long-Term Peace.” U.S. Army War College Senior Service College Fellowship Program Strategy Research Project. Carlisle Barracks: U.S. Army War College.

Wong, Edward. 2004. "Direct election of Iraq assembly pushed by cleric." The New York Times, January 12 .

Zoellick, Robert. 2010. “The key to rebuilding Afghanistan.” Washington Post, August 22. 


\section{Appendix}

\section{Figure A. 1: Map of the Democratic Republic of the Congo}

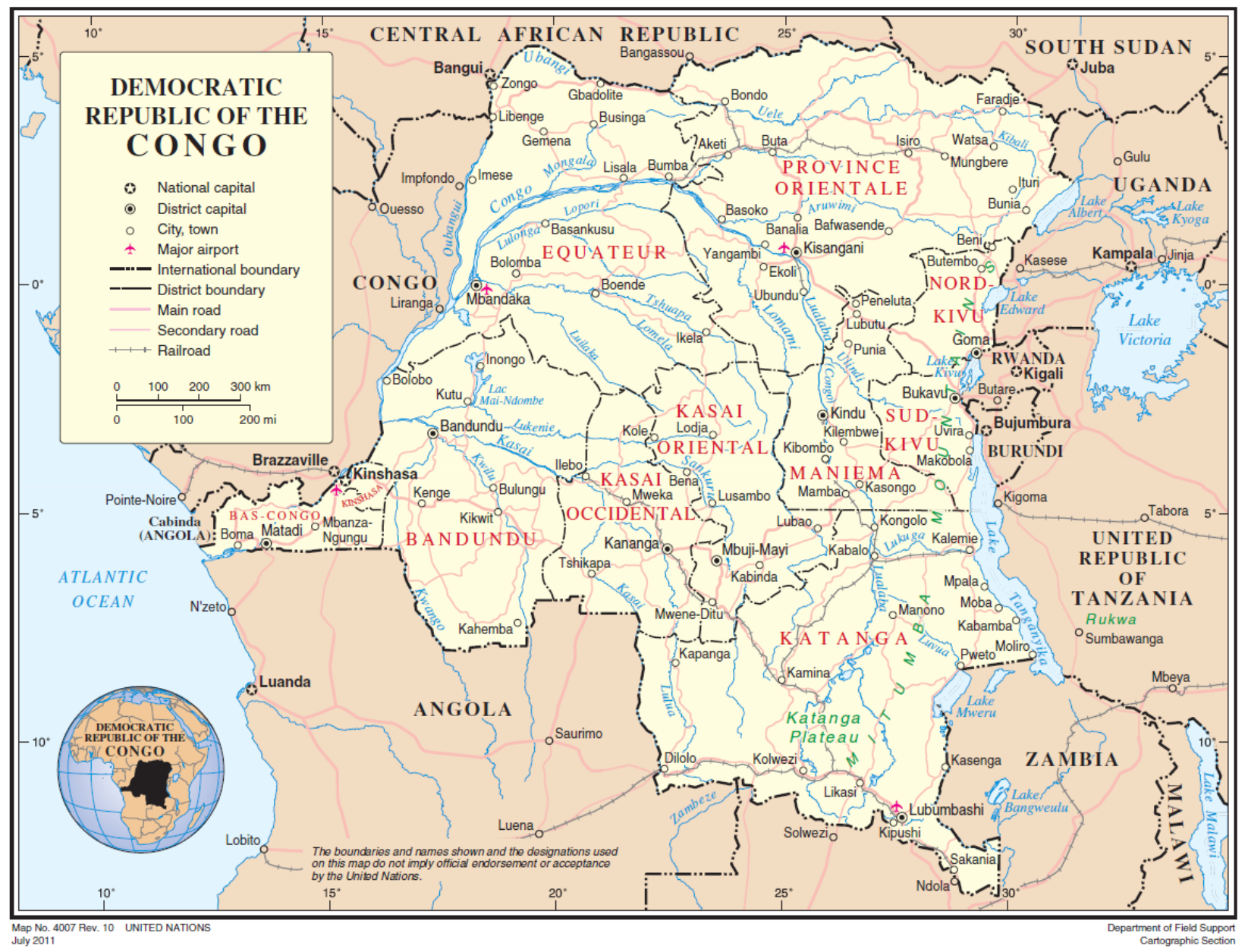

Source: United Nations, Department of Field Support, Cartographic Section, Map No. 4007, Revision 10, July 2011 